HU-EP-03/08

hep-th/0302221

\title{
Gauge Threshold Corrections in Intersecting Brane World Models
}

\author{
D. Lüst and S. Stieberger \\ Institut für Physik, Humboldt Universität zu Berlin, \\ Invalidenstraße 110, 10115 Berlin, FRG
}

\begin{abstract}
We calculate the one-loop corrections to gauge couplings in $\mathrm{N}=1$ supersymmetric brane world models, which are realized in an type $I I A$ orbifold/orientifold background with several stacks of D6 branes wrapped on 3-cycles with non-vanishing intersections. Contributions arise from both $\mathrm{N}=1$ and $\mathrm{N}=2$ open string subsectors. In contrast to what is known from ordinary orbifold theories, $\mathrm{N}=1$ subsectors do give rise to moduli-dependent one-loop corrections.
\end{abstract}




\section{Introduction}

Intersecting brane world models [1] have proven to be provide an interesting framework of getting string compactifications with promising phenomenological features. For the type IIA superstring these compactifications contain in particular several types of D6branes which are wrapped around 3-cycles of the internal space. Chiral fermions appear at intersections of branes at angles. The chiral fermion spectrum is determined by the topological intersection numbers of the relevant 3-cycles. Part of space-time supersymmetry is preserved if the D6-branes are wrapped around supersymmetric (special lagrangian) 3 -cycles, which are calibrated with respect to the same holomorphic 3-form as the O6planes are. This scenario was intensively investigated in the context of toroidal type IIA orientifolds and also for Calabi-Yau orientifolds, and in fact models with spectrum identical to the non-supersymmetric or supersymmetric Standard Model could be explicitly constructed. In a T-dual respectively mirror symmetric description one is dealing with D9-branes with additional gauge fluxes turned on. This can been seen very explicitly for the toroidal models after performing the T-duality transformation with respect to three internal directions. Then the various angles of the D6-branes translate themselves into constant magnetic gauge fluxes on the D9-branes, such that the internal torus becomes non-commutative.

In the present paper we turn to the question of computing one-loop gauge threshold corrections in intersecting brane world models, which is also very important from the phenomenological point of view. Unlike what happens e.g. in perturbative heterotic string vacua, the tree-level gauge couplings for the various gauge groups, arising from different stacks of branes, are not the same at the string scale. They follow from dimensional reducing the Born-Infeld action of a D6-brane on a 3-cycle of an internal six-torus $T_{6}$ and are essentially determined by the volume of the 3-cycle. E.g. for a six-torus $T_{6}=\prod_{j=1}^{3} T_{2}^{j}$ and a special 3-cycle embedded with the wrapping numbers $\left(n_{a}^{j}, m_{a}^{j}\right)$ w.r.t. to the two-tori $T_{2}^{j}$ the gauge couplings are given by $[2,3]$

$$
g_{a, \text { tree }}^{-2}=\frac{M_{\text {string }}^{3}}{2 \pi \lambda_{I I}} \prod_{j=1}^{3} \sqrt{\left(n_{a}^{j}\right)^{2}\left(R_{1}^{j}\right)^{2}+\left(m_{a}^{j}\right)^{2}\left(R_{2}^{j}\right)^{2}+2 n_{a}^{j} m_{a}^{j} R_{1}^{j} R_{2}^{j} \cos \alpha^{j}},
$$

with the type $I I$ coupling constant $\lambda_{I I}$ ( $c f$. the next section for more details). Hence a priori there is no unification of gauge couplings at the string scale (at string tree-level). One-loop gauge threshold corrections $\Delta_{a}$ (to the gauge group $G_{a}$ ), which take into account Kaluza-Klein and winding states from the internal dimensions and the heavy string modes,

1 The imaginary part of the standard $D=4$ dilaton field $S$ follows for $\left(n_{a}^{j}, m_{a}^{j}\right)=(1,0)$, i.e. $\operatorname{Im}(S)=g_{\text {string }}^{-2}=\frac{M_{\text {string }}^{3}}{2 \pi \lambda_{I I}} R_{1}^{1} R_{1}^{2} R_{1}^{3}$. 
may change this picture [4. For certain regions in moduli space these corrections may become huge and thus have a substantial impact 2 on the unification scale. One-loop gauge corrections are very important quantities to probe the low-energy physics below the string scale as they change the running of the gauge couplings for scales $\mu$ below the string scale according to the Georgi, Quinn and Weinberg evolution equations of ordinary field theories:

$$
\frac{1}{g_{a}^{2}(\mu)}=\frac{1}{g_{a, \text { tree }}^{2}}+\frac{b_{a}}{16 \pi^{2}} \ln \frac{M_{\text {string }}^{2}}{\mu^{2}}+\Delta_{a} .
$$

In the type IIA picture with intersecting D6-branes these threshold correction $\Delta_{a}$ will depend on the holomolgy classes on the 3-cycles (open string parameters) and also on the closed string geometrical moduli. In toroidal models these corrections will be given in terms of the wrapping numbers $n_{a}^{j}, m_{a}^{j}$ and the radii $R_{i}^{j}$ of the torus, in analogy to their tree-level counterparts (1.1), however in a more complicated way. In the equivalent T-dual picture the threshold corrections will be a function of the open string magnetic gauge fluxes and of the geometrical moduli of the dual compact space. Since the gauge fluxes are directly related to the non-commutativity parameters of the internal torus, we obtain in this way some interesting, new informations for one-loop threshold corrections on non-commutative tori in string theory, a discussion which extends recent results on one-loop corrections on compact non-commutative spaces in the literature [9] and will be further discussed in [10].

In this article we shall calculate the quantities $\Delta_{a}$ for a class of realistic brane world models realized through intersecting branes which are wrapped on internal tori. We mainly focus on supersymmetric intersecting brane models and leave the discussion of the nonsupersymmetric case for a future publication [10]. The main motivation to discuss supersymmetric models is, that in these theories vacuum $R$-tadpoles and therefore also vacuum $N S$-tadpoles are cancelled, as the tensions of the D-branes and of the orientifold planes precisely balance each other. As we will show this guarantees also the absence of both $R$ - and $N S$-tadpoles for one-loop gauge couplings, thus providing $U V$-finite results for these corrections. On the other hand one-loop gauge corrections in non-supersymmetric intersecting brane models are plugged by $U V$-divergences. Ultimatively however, one shall be interested in non-supersymmetric models. But it seems more convenient, to start with a supersymmetric intersecting brane model, in which at least certain couplings are free of $U V$-divergences and then breaks supersymmetry by some mechanism, which does not spoil 3 the $U V$-finiteness of the couplings. As the main result of this paper we will show

2 This effect has been thoroughly investigated for heterotic $N=1$ string vacua in [5] [7] and will be discussed for the models under discussion elsewhere [8].

3 This procedure is possible on the heterotic side, where world-sheet modular invariance guarantees $U V$-finiteness even after supersymmetry breaking. See Ref. [11] for a detailed discussion on this problem. 
that the threshold corrections in $\mathrm{N}=1$ sectors of intersecting brane world models exhibit a very interesting, new moduli dependence which is in contrast to the one-loop threshold correstions of heterotic $\mathrm{N}=1$ sectors, which are moduli independent.

After a short introduction into the construction of these models we work out in section 2 the background gauge dependence of the partition function of the open string sectors. After a brief review of the technical aspects of orientifolds with supersymmetric intersecting branes, we discuss in subsection 3.1. for a given gauge group the possible contributions from the various open string diagrams to its one-loop correction. These contributions, originating from so-called $\mathrm{N}=1$ and $\mathrm{N}=2$ supersymmetric sectors, will be calculated in subsections 3.2. and 3.3, respectively. The calulation for the $\mathrm{N}=1$ sectors need some excursion into the mathematical problem of how to extract Dirichlet series from modular forms. This allows us to perform certain Schwinger type integrals over Eisenstein series, presented in appendix A. In addition we shall need some useful spin-structure sums of the gauged open the string partition function, presented in appendix B. In subsection 3.4 we prove the finiteness of our one-loop gauge threshold results. This needs the UV-limits of the gauged open string partition functions, which we exhibit in appendix C. We shall see, that in the case of non-anomalous gauge groups only NS-tadpoles have to be cancelled. The explicit moduli dependence of our $\mathrm{N}=1$ threshold result, given through the radii $R_{1}^{i}, R_{2}^{i}$ rather than the angles of the branes, is shown in subsection 3.6. In subsection 3.7 we apply our results from subsections 3.2 and 3.3 to write down the one-loop gauge corrections for a $\mathbf{Z}_{2} \times \mathbf{Z}_{2}$ orientifold example with stacks of intersecting branes.

\section{Intersecting branes and gauged open string partition functions}

In subsection 2.1 we review general aspects of intersecting branes in IIA toroidal compactifications, which take over to orientifold constructions without or with orbifold twists. Some important technical details of the latter, which represent more general brane constructions, in particular with the possibility of having $\mathrm{N}=1$ space-time supersymmetry, will be reviewed in subsection 2.2. The main result of this section are the partition functions for open strings stretched between intersecting branes in the presence of a (space-time) magnetic field. They will be derived in subsection 2.3. These formulae are quite general and may be used for intersecting branes both in toroidal and orientifold/orbifold compactifications of type II string theory with arbitrary amount of supersymmetry. 


\subsection{Toroidal compactification of intersecting branes}

We consider configurations of type II D6 branes wrapped on non-trivial three-cycles of a six-dimensional torus $T^{6}$. The gauge theories on the four non-compact dimensions of the brane world-volume are generically non-chiral and non-supersymmetric, whereas the bulk, where all closed string modes live, preserves all 32 supersymmetries of type II. However placing intersecting branes into an orientifold background ( $c f$. Section 3 for more details) allows for both non-chiral and chiral supersymmetric gauge theories. The torus is taken to be a direct product $T_{6}=\prod_{j=1}^{3} T_{2}^{j}$ of three two-dimensional tori $T_{2}^{j}$ with radii $R_{1}^{j}, R_{2}^{j}$ and angles $\alpha^{j}$ w.r.t. to the compact dimensions $e_{1}^{j}, e_{2}^{j}$. The Kähler and complex structure modulus of these tori are defined as usual:

$$
U^{j}=\frac{R_{2}^{j}}{R_{1}^{j}} e^{i \alpha^{j}}, T^{j}=b^{j}+i R_{1}^{j} R_{2}^{j} \sin \alpha^{j},
$$

with the torus $B$-field $b^{j}$. Furthermore, the three-cycle is assumed to be a factorizable into a direct product of three one-cycles, each of them wound around a torus $T_{2}^{j}$ with the wrapping numbers $\left(n^{j}, m^{j}\right)$ w.r.t. the fundamental 1 -cycles of the torus. Hence the angle of the $D 6$-brane with the $Y_{1}^{j}$-axis is given by

$$
\tan \phi^{j}=\frac{m^{j} R_{2}^{j}}{n^{j} R_{1}^{j}} .
$$

Generally, two branes with wrapping numbers $\left(n_{a}^{j}, m_{a}^{j}\right)$ and $\left(n_{b}^{j}, m_{b}^{j}\right)$, are parallel in the subspace $T_{2}^{j}$, if their intersection number

$$
I_{a b}^{j}=\operatorname{det}\left(\begin{array}{cc}
n_{a}^{j} & m_{a}^{j} \\
n_{b}^{j} & m_{b}^{j}
\end{array}\right)
$$

w.r.t. to this subspace vanishes, $I_{a b}^{j}=0$. For later convenience let us also introduce:

$$
\pi v^{j}:=\operatorname{arctanh}\left(\mathcal{F}^{j}\right)
$$

which implies $\phi^{j}=i \pi v^{j}$. Chiral fermions appear at (non-vanishing) intersections of two D6-branes.

In the $T$-dual picture, the $D 6$-branes at angles $\phi^{j}$ are mapped to $D 9$-branes with magnetic fluxes or background gauge fields $F^{j}$. Thereby the gauge field (magnetic flux) $F^{j}$ on the brane is related to the angles (2.2) through:

$$
\frac{m^{j}}{n^{j} R_{1}^{j} R_{2}^{j}}=: i F^{j}
$$

which results from (2.2) by a $T$-duality in all $Y_{2}^{j}$-directions. 


\subsection{Some technical aspects of intersecting branes in orbifold/orientifold backgrounds}

The condition for tadpole cancellations in II A orientifold backgrounds in four spacetime dimensions requires a system of $D 6$ branes which has to respect the orbifold and orientifold projections. In particular this means, that in addition to each brane a system of mirror pairs have to be introduced. In the models, discussed in [12, 13], the $D 6$ branes are located along each orientifold plane and tadpoles are cancelled locally. This leads to a non-chiral $\mathrm{N}=1$ spectrum in $D=4$. However, as realized in [14, 15], this setup may be relaxed by placing the $D 6$ branes at angles w.r.t. only one orientifold plane. In addition, for consistency with the orbifold/orientifold group their orbifold/orientifold mirrors have to be introduced. In doing so, it is possible to cancell (non-locally) tadpoles from several orientifold planes with fewer branes than if one placed branes on top of each orientifold plane. The requirement of $R$-tadpole cancellation leads to some constraints on the number and location of the $D 6$ branes. Further restrictions arise in the case of space-time supersymmetry, where $N S$ vacuum tadpole cancellation follows from $R$ vacuum tadpole cancellation. One main advantage of allowing for branes with non-vanishing angle w.r.t. to the orientifold plane is the possibilty of a chiral $\mathrm{N}=1$ spectrum.

Intersecting brane world models with $\mathrm{N}=1$ supersymmetry in $D=4$ have been introduced in [12, 13, 16, 17] through orbifold/orientifold projection. The orientifold action $\Omega \mathcal{R}$ in theses models is a combination of reversal of world-sheet parity $\Omega$ and a reflection of the three internal coordinates:

$$
\mathcal{R}: Y^{2,4,6} \rightarrow-Y^{2,4,6} .
$$

The orbifold group is generated by elements from $\mathbf{Z}_{N}$ (or $\mathbf{Z}_{N} \times \mathbf{Z}_{M}$ ). The latter are represented by the $\theta$ (and $\omega$ ), describing discrete rotations on the compact coordinates $Y^{i}$. This action restricts the compactification lattice and fixes some of the internal parameter (2.1) to discrete values. The orientifold $O 6$-planes describe the set of points which are invariant under the group actions $\Omega \mathcal{R}, \Omega \mathcal{R} \theta^{k}, \Omega \mathcal{R} \omega^{l}$ and $\Omega \mathcal{R} \theta^{k} \omega^{l}$. These planes are generated by rotations of the real $Y^{2 i-1}$ axes by $\theta^{-k / 2} \omega^{-l / 2}$ and will be denoted by $O 6_{k ; l}$ in the following.

The models we shall discuss consist of several stacks $a$ of $D 6$-branes. To each stack, consisting of $N_{a}$ parallel $D 6$ branes, a specific gauge group 1 t $G_{a}$ is associated. In addition, in orbifold/orientifold backgrounds, there must exist to each stack $a$ a set of orbifold mirrors $\theta^{n} a$ and orientifold $\Omega \mathcal{R}$-mirrors thereof in order to be consistent with the orbifold and orientifold group. This way we obtain an array of $D$-branes at angles, if the stacks go

4 Before the orientifold and orbifold projection the gauge group is $U\left(N_{a}\right)$ in the case, when the branes are not parallel to an orientifold plane within some subspace. Otherwise the gauge group is $U S p\left(N_{a}\right)$ or $S O\left(N_{a}\right)$. 
through a fixpoint of the orbifold group, the case we shall consider here. Hence, following the terminology of [15], any stack $a$ is organized in orbits, which represent an equivalence class $[a]$. For $N, M \neq 2$ the length of each orbit $[a]$ is at most $2 N M$, but may be smaller, if e.g. stack $a$ is located along an orientifold plane. In that case the length of the orbit is only $M N$. Since a $\mathbf{Z}_{2}$ orbifold twist leaves a brane invariant, in the case of $N, M=2$, the class $[a]$ consists of just two elements. Stacks within a conjugacy class $[a]$ have non-trivial intersections among each other and w.r.t. to stacks from a different class $[b]$ belonging to the gauge group $G_{b}$.

The orientifold and orbifold group lead to various open string sectors describing strings starting and ending on the $D$-branes. The way, how all these sectors contribute to the vacuum partition function is highly determined by the requirement of vacuum tadpole cancellation. In addition some conditions on the representation of the orientifold group on the Chan-Paton indices follow. Open strings starting on a brane from $a$ and ending on one of its orbifolded mirrors $\theta^{n} a$ belong to the so-called open string $\theta^{2 n}$-twisted sector. In addition, as known from closed string untwisted orbifold sectors, there exist twist insertions $\theta^{k}$ in the partition function, which restricts the contributions to the spectrum to $\theta^{k}$ invariant states. This means, that in an annulus diagram $A_{a a^{\prime}}^{k}$, which describes an $\theta^{2 n}$-twisted open string starting on stack $a$ and ending on its mirror $a^{\prime}=\theta^{n} a$, the two stacks $a$ and $a^{\prime}$ and the orientation of the open string have to be invariant under $\theta^{k}$. In addition we have open string exchanges $A_{a b}^{k}$ between branes from different classes $[a]$ and $[b]$, supplemented with the twist insertion $\theta^{k}$. Its is evident, that for branes sitting not at orientifold planes a $\theta^{k}$ twist insertion is only possible, if $\theta^{k}$ represents a $\mathbf{Z}_{2}$ twist, i.e. for $\theta^{N / 2}, \omega^{M / 2}$ in the case of even order orbifolds. However, in [12,13] it has been shown, that this statement holds also for branes sitting at orientifold planes. Generically open string annulus diagrams with $\mathbf{Z}_{2}$ twist insertions lead to twisted sector tadpoles in the closed string channel. The latter describing an exchange of a twisted 5-form field cannot be cancelled by other amplitudes. Therefore one imposes a twisted sector tadpole cancellation condition on the $\gamma_{\theta^{k}}^{a}$-matrices acting on the Chan-Paton factors of the open string ends [12,13]:

$$
\operatorname{Tr} \gamma_{\theta^{N / 2}}^{a}=0, \operatorname{Tr} \gamma_{\omega^{M / 2}}^{a}=0, \quad \operatorname{Tr} \gamma_{\theta^{M / 2} \omega^{M / 2}}^{a}=0 .
$$

This conditions ensure, that we have not to further discuss those sectors in the vacuum partition function, and we drop the subscript $k$ on $A_{a a^{\prime}}^{k}, A_{a b}^{k}$. We shall show in section 3 , that this statements takes over for the calculation of the one-loop gauge couplings. The complete annulus partition function takes the form

$$
A=\sum_{a, b=1}^{K} N_{a} N_{b} \sum_{\substack{a \in[a] \\ b \in[b], a \neq b}} A_{a b}=\sum_{a=1, \ldots, K} N_{a} \sum_{\substack{b=1 \\ b \neq a}}^{K} N_{b} \sum_{\substack{a \in[a] \\ b \in[b]}} A_{a b}+\sum_{a=1}^{K} N_{a}^{2} \sum_{\substack{a, a^{\prime} \in[a] \\ a \neq a^{\prime}}} A_{a a^{\prime}}
$$


with the model dependent numbers $a_{a b}$, which may looked up from Refs. [12, 13, 16]. The numbers $N_{a}, N_{b}$ arise from the traces over the $\gamma$-matrices acting on the Chan-Paton factors $\left(\gamma_{1}^{a}=\mathbf{1}_{N_{a}}\right)$ :

$$
\operatorname{Tr} \gamma_{1}^{a}=N_{a}
$$

We divided the sums into contributions from open string exchanges within one conjugacy class $[a]$ and exchanges between stacks from different conjugacy classes $[a]$ and $[b]$. In the next subsection we shall see, that this split turns out to be important to disentangle the various contributions to the one-loop gauge corrections.

Contributions to the Möbius partition function may come from untwisted and twisted sectors with insertion $\Omega \mathcal{R} \theta^{k}$. In both cases the whole arrangement has to be invariant under the action of $\Omega \mathcal{R} \theta^{k}$. An untwisted Möbius diagram $M_{a, a}^{k}$ with insertion $\Omega \mathcal{R} \theta^{k}$ describes a string starting and ending on stack $a$. Because of the insertion $\Omega \mathcal{R} \theta^{k}$, this exchange may be only possible, if stack $a$ sits on the orientifold plane $O 6_{\Omega \mathcal{R} \theta^{k}}:=O 6_{k}$ (see the examples in Ref. [12,13]). A Möbius diagram accounting for a string starting from stack $a$ and ending on its orientifold image $\Omega \mathcal{R} a$ admits only the twist insertions 1 or $\theta^{N / 2}$, provided stack $a$ does not sit on an orientifold plane. We shall denote these two diagrams by $M_{a, \Omega \mathcal{R} a}^{0}$ and $M_{a, \theta^{N / 2} \Omega \mathcal{R} a}^{N / 2}$, respectively. Restrictions on the allowed twist insertion $\theta^{k}$ follow also for twisted Möbius diagrams. Namely, only the combinations $M_{a, \Omega \mathcal{R} \theta^{k} a}^{k}$ and $M_{\Omega \mathcal{R} a, \theta^{N-k} a}^{k}$ represent twist invariant open string exchanges. The brane $\Omega \mathcal{R} \theta^{k} a$ arises from a reflection of brane $a$ on the orientifold plane $O 6_{k}$. As we shall see in subsection 2.4 these combinations lead to only untwisted sectors after transforming into the closed string channel. This is in agreement, that only untwisted closed strings interact with the crosscaps. With $\Omega \mathcal{R} \theta^{k}=\theta^{N-k} \Omega \mathcal{R}$, we may express all possible Möbius contributions the vacuum partition function by the following sum

$$
M=\sum_{a=1}^{K} N_{a} \sum_{a \in[a]} \sum_{k=0}^{N-1} \rho_{\Omega \mathcal{R} \theta^{k}} M_{a, \Omega \mathcal{R} \theta^{k} a}^{k},
$$

with the phases $\rho_{\Omega \mathcal{R} \theta^{k}}$ as a result from taking the trace

$$
\operatorname{Tr}\left[\left(\gamma_{\Omega \mathcal{R} \theta^{k}}^{\Omega \mathcal{R}}\right)^{*} \gamma_{\Omega \mathcal{R} \theta^{k}}^{a}\right]=\rho_{\Omega \mathcal{R} \theta^{k}} N_{a}
$$

over the matrices $\gamma_{\Omega \mathcal{R} \theta^{k}}^{a}, \gamma_{\Omega \mathcal{R} \theta^{k}}^{\Omega \mathcal{R} \theta^{k}}$ representing the twist action $\Omega \mathcal{R} \theta^{k}$ on brane $a$ and $\Omega \mathcal{R} \theta^{k} a$, respectively. The latter relation generally holds for the Chan-Paton matrices in orbifold/orientifold compactifications thanks to the relation: $\left(\gamma_{\Omega \mathcal{R} \theta^{k}}^{\Omega \mathcal{R}}\right)^{k} \gamma_{\Omega \mathcal{R} \theta^{k}}^{a}=\rho_{\Omega \mathcal{R} \theta^{k}} \gamma_{1}^{a}=$ $\rho_{\Omega \mathcal{R} \theta^{k}} \mathbf{1}_{N_{a}}$.

5 To keep the formulae readable we only display the Möbius sector for $\mathbf{Z}_{N}$ orbifolds. The changes to be made for $\mathbf{Z}_{N} \times \mathbf{Z}_{M}$ orbifolds are straightforward. 


\subsection{Gauging the open string partition function}

All branes we have described above have in common their four-dimensional (noncompact) Minkowski space. Hence their gauge fields are located on parallel fourdimensional subspaces, which may be seperated (in the cases $I_{a b}^{j} \neq 0$ and $I_{a a^{\prime}}^{j} \neq 0$ ) in the transverse internal dimensions. One-loop corrections to the gauge couplings are realized through exchanges of open strings in that transverse space. The open string charges $q_{a}, q_{b}$ at their ends couple to the external gauge fields sitting on the branes. Only annulus $A$ and Möbius $M$ diagrams contribute, as torus and Klein bottle diagrams refer to closed string states.

When we consider the one-loop correction to the gauge group $G_{a}$, at least one open string end has always to be charged under the gauge group $G_{a}$, i.e. at least one open string end must always end on a brane from stack $a$ (or from its mirrors $\theta^{n} a, \Omega \mathcal{R} \theta^{k} a, \ldots$ ). Open string exchanges between parallel branes preserve $\mathrm{N}=4$ supersymmetry in the case of no twist insertion. Therefore, neither $A_{a a}$ nor $M_{a a}^{0}$ give rise to gauge coupling renormalization.

Three different kinds of open string exchanges are possible: An (annulus) exchange $A_{a b}$ between stack $a \in[a]$ constituing to the gauge group $G_{a}$ and stack $b \in[b]$ belonging to the gauge group $G_{b}$. Second, we have to consider (annulus) open string exchanges $A_{a a^{\prime}}$ between two stacks $a$ and $a^{\prime}$ from the conjugacy class $[a]$ associated to the same gauge group $G_{a}$. Since $a \neq a^{\prime}$ this exchange generically belongs to the open string twisted sector. Third, there is the whole set of Möbius diagrams referring to stack $a$ and showing up in the sum (2.10) for $a$.

After having presented formally the various kinds of open string diagrams relevant for one-loop gauge couplings in orbifold/orientifold backgrounds with intersecting branes, we shall now discuss their gauge background dependent partition functions. We shall compute the one-loop corrections to the gauge couplings by the background field method: We turn on a (space-time) magnetic field, e.g. $F_{23}=B Q_{a}$ in the $X^{1}$-direction and determine the dependence of the open string partition function on that field. Here, $Q_{a}$ is an appropriatley normalized generator of the gauge group $G_{a}$ under consideration. The second order of an expansion w.r.t. to $B$ of the gauged partition function gives the relevant piece for the one-loop gauge couplings. This procedure has been previously already applied in [18,19] to obtain one-loop gauge threshold corrections in certain type $I$ orientifold compactifications. The presence of an external (space-time) gauge field strength $F:=F_{23}=B Q_{a}$, which couples to the charges $6 q_{a}, q_{a^{\prime}}$ of the open string ends, results in an shift of the open string oscillator modes by the amount [20]:

$$
\pi \epsilon=\arctan \left(\pi q_{a} B\right)+\arctan \left(\pi q_{a^{\prime}} B\right)
$$

6 More precisely, $q_{a}, q_{a^{\prime}}$ are the eigenvalues of the group generator $Q_{a}$ acting on the ChanPaton states of both string ends. 
This shift modifies the event spin-structure part of the open string space-time partition function in the following way [21]

$$
\begin{aligned}
\frac{1}{\eta(\tau)^{2}} \frac{\theta\left[\begin{array}{l}
\delta_{1} \\
\delta_{2}
\end{array}\right](0, \tau)}{\eta(\tau)} & i \text { it } \beta \frac{\theta\left[\begin{array}{l}
\delta_{1} \\
\delta_{2}
\end{array}\right]\left(\frac{i \epsilon t}{2}, \tau\right)}{\theta\left[\begin{array}{l}
1 / 2 \\
1 / 2
\end{array}\right]\left(\frac{i \epsilon t}{2}, \tau\right)} \\
& = \begin{cases}i t \beta e^{-2 \pi i \epsilon\left(\delta_{2}-\frac{1}{2}\right)} \frac{\theta\left[\begin{array}{c}
\delta_{1}+\epsilon \\
\delta_{2}
\end{array}\right](0, \tau)}{\theta\left[\begin{array}{c}
\frac{1}{2}+\epsilon \\
\frac{1}{2}
\end{array}\right](0, \tau)} & , \tau=\tau_{A}=\frac{i t}{2} \\
i t \beta e^{-2 \pi i \epsilon\left(\delta_{2}-\frac{1}{2}\right)} \frac{\theta\left[\begin{array}{c}
\delta_{1}+\epsilon \\
\delta_{2}-\frac{\epsilon}{2}
\end{array}\right](0, \tau)}{\theta\left[\begin{array}{c}
\frac{1}{2}+\epsilon \\
\frac{1}{2}-\frac{\epsilon}{2}
\end{array}\right](0, \tau)} & , \tau=\tau_{M}=\frac{i t}{2}+\frac{1}{2}\end{cases}
\end{aligned}
$$

with:

$$
\beta=\pi B\left(q_{a}+q_{a^{\prime}}\right) .
$$

Here, $\tau_{A}$ and $\tau_{M}$ are the modular parameter of the annulus and Möbius open string partition functions, respectively. In the Möbius amplitude $M_{a}$ the two endpoint charges are the same, while for the annulus amplitude $A_{a b}$ describing an open string exchange between one stack of gauge group $G_{a}$ an an other stack of different gauge group $G_{b}$ the second charge is zero (under the $B$-field under consideration). Thus we have for the net oscillator shifts (2.12):

$$
\begin{aligned}
A_{a b}: & \pi \epsilon_{a b}=\arctan \left(\pi q_{a} B\right), \\
A_{a a^{\prime}}: & \pi \epsilon_{a a^{\prime}}=\arctan \left(\pi q_{a} B\right)+\arctan \left(\pi q_{a^{\prime}} B\right), \\
M_{a}: & \pi \epsilon_{a}=2 \arctan \left(\pi q_{a} B\right) .
\end{aligned}
$$

In addition we obtain for (2.14):

$$
\begin{aligned}
A_{a b}: & \beta_{a b}=\pi B q_{a} \\
A_{a a^{\prime}}: & \beta_{a a^{\prime}}=\pi B\left(q_{a}+q_{a^{\prime}}\right) \\
M_{a}: & \beta_{a}=2 \pi B q_{a} .
\end{aligned}
$$

Now we have collected all details to gauge the partition function of an open string stretched between two intersecting stacks $a$ and $b$ ( $a^{\prime}$, respectively) with rotation angles $\phi_{a}^{j}$ and $\phi_{b}^{j}\left(\phi_{a^{\prime}}^{j}\right.$, respectively) w.r.t. the three complex tori $T_{2}^{j}$. The latter have been presented in 14, however may be easily obtained by applying the procedure (2.13) also on the internal open string coordinates:

7 The modification in the odd part of the partition function is more involved due to the presence of fermionic zero modes. Eventually we are interested in $C P$ even gauge couplings. Hence we do not have to worry about gauging odd fermions. 
The gauged open string partition function $A_{a b}^{d}$ for an open string stretched between a stack $a$ associated to the gauge group $G_{a}$ under consideration and a stack $b$ of different gauge group becomes:

$$
\begin{aligned}
A_{a b}^{d}(B) & =i^{d+1} \beta_{a b} t^{d+d^{\prime}-4} \prod_{i=1}^{d^{\prime}} Z_{i}\left(t, T^{i}, V^{i}\right) \\
& \times \sum_{\vec{\delta}} s_{\vec{\delta}} \frac{\theta\left[\begin{array}{l}
\delta_{1} \\
\delta_{2}
\end{array}\right]\left(\frac{i \epsilon_{a b} t}{2}, \frac{i t}{2}\right)}{\theta\left[\begin{array}{l}
1 / 2 \\
1 / 2
\end{array}\right]\left(\frac{i \epsilon_{a b} t}{2}, \frac{i t}{2}\right)} \frac{\theta\left[\begin{array}{l}
\delta_{1} \\
\delta_{2}
\end{array}\right]\left(0, \frac{i t}{2}\right)^{3-d}}{\eta\left(\frac{i t}{2}\right)^{9-3 d}} \prod_{j=1}^{d} I_{a b}^{j} \frac{\theta\left[\begin{array}{l}
\delta_{1} \\
\delta_{2}
\end{array}\right]\left(\frac{v_{a b}^{j} t}{2}, \frac{i t}{2}\right)}{\theta\left[\begin{array}{l}
1 / 2 \\
1 / 2
\end{array}\right]\left(\frac{v_{a b}^{j} t}{2}, \frac{i t}{2}\right)} .
\end{aligned}
$$

The spin structure sum runs over even spin-structures $\vec{\delta}=\left(\delta_{1}, \delta_{2}\right)$, only. Their phases are given by $s_{(0,0)}=1, s_{\left(0, \frac{1}{2}\right)}=s_{\left(\frac{1}{2}, 0\right)}=-1$. The quantity $v_{a b}^{j}(c f$. (2.4)) decribes the relative angle of brane $a$ and $b$

$$
v_{a b}^{j}:=v_{b}^{j}-v_{a}^{j}=\frac{i}{\pi}\left(\phi_{a}^{j}-\phi_{b}^{j}\right)
$$

in close analogy to what happens in the space-time in the presence of the background magnetic field $B(c f .(2.12))$. Furthermore, the supscript $d=0, \ldots, 3$ denotes the number of internal tori, in which the brane $a$ and $b$ have non-vanishing intersection $I_{a b}^{j} \neq 0$, i.e. $v_{a b}^{j} \neq$ $0, \pm i$. In the remaining $d^{\prime}$ complex internal coordinates they have vanishing intersections $v_{a b}^{i}=0, \pm i$. Hence w.r.t. these tori $T_{2}^{i}$ the open strings have non-vanishing Kaluza-Klein momenta and windings. Their mass is given by the mass formula of open strings stretched between two parallel $D 1$ branes, which are wrapped around the torus $T_{2}^{i}$ with wrapping numbers $\left(n_{a}^{i}, m_{a}^{i}\right)$. Their (zero mode) partition function has been determined in [22, 23]

$$
Z_{i}\left(t, T^{i}, V_{a}^{i}\right)=\sum_{r, s \in \mathbf{Z}} e^{-\frac{\pi t}{T_{2}^{i} V_{a}^{i}}\left|r+T^{i} s\right|^{2}}
$$

with the moduli (2.1) and:

$$
V_{a}^{i}=\frac{1}{U_{2}^{i}}\left|n_{a}^{i}+U^{i} m_{a}^{i}\right|^{2}=\left[\frac{R_{1}^{i}}{R_{2}^{i}}\left(n_{a}^{i}\right)^{2}+\frac{R_{2}^{i}}{R_{1}^{i}}\left(m_{a}^{i}\right)^{2}+2 n_{a}^{i} m_{a}^{i} \cos \alpha^{i}\right] \frac{1}{\sin \alpha^{i}} .
$$

Here and in the following $n_{a}^{i}$ and $m_{a}^{i}$ are assumed to be coprime integers in order to avoid multiple wrappings. Since we focus on intersecting $D 6$ branes with a four-dimensional Minkowskian space we have $d+d^{\prime}=3$. For the annulus amplitude $A_{a a^{\prime}}^{d}$ describing open strings starting and ending on stacks $a$ and $a^{\prime}$ within the same orbit $[a]$ and thus belonging to the gauge group $G_{a}$ we obtain:

$$
\begin{aligned}
A_{a a^{\prime}}^{d}(B) & =i^{d+1} \beta_{a a^{\prime}} B t^{d+d^{\prime}-4} \prod_{i=1}^{d^{\prime}} Z_{i}\left(t, T^{i}, V^{i}\right) \\
& \times \sum_{\vec{\delta}} s_{\vec{\delta}} \frac{\theta\left[\begin{array}{l}
\delta_{1} \\
\delta_{2}
\end{array}\right]\left(\frac{i \epsilon_{a a^{\prime}} t}{2}, \frac{i t}{2}\right)}{\theta\left[\begin{array}{l}
1 / 2 \\
1 / 2
\end{array}\right]\left(\frac{i \epsilon_{a a^{\prime}} t}{2}, \frac{i t}{2}\right)} \frac{\theta\left[\begin{array}{l}
\delta_{1} \\
\delta_{2}
\end{array}\right]\left(0, \frac{i t}{2}\right)^{3-d}}{\eta\left(\frac{i t}{2}\right)^{9-3 d}} \prod_{j=1}^{d} I_{a a^{\prime}}^{j} \frac{\theta\left[\begin{array}{l}
\delta_{1} \\
\delta_{2}
\end{array}\right]\left(\frac{v_{a a^{\prime}}^{j}}{2}, \frac{i t}{2}\right)}{\theta\left[\begin{array}{l}
\frac{1}{2} \\
\frac{1}{2}
\end{array}\right]\left(\frac{v_{a a^{\prime}}^{j}}{2}, \frac{i t}{2}\right)} .
\end{aligned}
$$


As before $(c f$. (2.18) $), v_{a a^{\prime}}^{j}$ is given by the relative angle of the two branes $a$ and $a^{\prime}$ :

$$
v_{a a^{\prime}}^{j}:=v_{a^{\prime}}^{j}-v_{a}^{j}=\frac{i}{\pi}\left(\phi_{a}^{j}-\phi_{a^{\prime}}^{j}\right) .
$$

Furthermore, for the gauged Möbius amplitude $M_{a, \Omega \mathcal{R} \theta^{k} a}^{k ; d}(B)$ describing an open string starting on a generic stack $a \in[a]$ of the gauge group $G_{a}$ and ending on its orientifold mirror $\Omega \mathcal{R} \theta^{k} a$ supplemented with the twist insertion $\theta^{k}$, we obtain:

$$
\begin{aligned}
M_{a, \Omega \mathcal{R} \theta^{k} a}^{k ; d}(B) & =-i^{d+1} \beta_{a} t^{d+d^{\prime}-4} \prod_{i=1}^{d^{\prime}} n_{O 6_{k}}^{i} L_{i}\left(t, T^{i}, V_{O 6_{k}}^{i}\right) \sum_{\vec{\delta}} s_{\vec{\delta}} \frac{\theta\left[\begin{array}{l}
\delta_{1} \\
\delta_{2}
\end{array}\right]\left(\frac{i \epsilon_{a} t}{2}, \frac{i t}{2}+\frac{1}{2}\right)}{\theta\left[\begin{array}{l}
1 / 2 \\
1 / 2
\end{array}\right]\left(\frac{i \epsilon_{a} t}{2}, \frac{i t}{2}+\frac{1}{2}\right)} \\
& \times \prod_{i=1}^{d^{\prime}} \frac{\theta\left[\begin{array}{l}
\delta_{1} \\
\delta_{2}
\end{array}\right]\left(0, \frac{i t}{2}+\frac{1}{2}\right)^{3-d}}{\eta\left(\frac{i t}{2}+\frac{1}{2}\right)^{9-3 d}} \prod_{j=1}^{d} 2^{\delta_{j}} \frac{I_{a, \Omega \mathcal{R} \theta^{k} a}^{k ; j} \theta\left[\begin{array}{l}
\delta_{1} \\
\delta_{2}
\end{array}\right]\left(v_{a}^{k ; j} t, \frac{i t}{2}+\frac{1}{2}\right)}{\theta\left[\begin{array}{l}
\frac{1}{2} \\
\frac{1}{2}
\end{array}\right]\left(v_{a}^{k ; j} t, \frac{i t}{2}+\frac{1}{2}\right)} .
\end{aligned}
$$

Here, $v_{a}^{k ; j}$ is related to the angle $\phi_{a}^{k ; j}$ between brane $a$ and the orientifold plane $O 6_{k}$ through:

$$
v_{a}^{k ; j}=-\frac{i}{\pi} \phi_{a}^{k ; j}=-\frac{i}{\pi}\left(\phi_{a}^{j}-\phi_{O 6_{k}}^{j}\right) .
$$

The twist insertion $\theta^{k}$ is automatically respected by choosing $v_{a}^{k ; j}$ in that way. Of course, for $k=0$, we just have $\phi_{a}^{0 ; j}=\phi_{a}^{j}$, with $\phi_{a}^{j}$ being the angle of brane $a$ w.r.t. to the positive axis $Y^{2 j-1}$. The factor $2^{\delta_{j}}$ is an important correction [12], relevant if brane $a$ is orthogonal to the orientifold plane $O 6_{k}$ in the torus $T_{2}^{j}$, i.e. $\delta=1$ for $\phi_{a}^{k ; j}= \pm \frac{\pi}{2}$. The intersection number $I_{a, \Omega \mathcal{R} \theta^{k} a}^{k j j}$ counts the number of $\Omega \mathcal{R} \theta^{k}$-invariant intersections of the two branes $a$ and $a^{\prime}=\Omega \mathcal{R} \theta^{k} a$

$$
I_{a ; \Omega \mathcal{R} \theta^{k} a}^{k ; j}:=I_{a ; O 6_{k}}^{j}=\left(n_{a}^{j} m_{O 6_{k}}^{j}-m_{a}^{j} n_{O 6_{k}}^{j}\right),
$$

with $\left(n_{O 6_{k}}^{j}, m_{O 6_{k}}^{j}\right)$ characterizing the orientifold plane $O 6_{k}$ w.r.t. to the torus $T_{2}^{j}$. In particular we have [24,16,25]

$$
I_{a, \Omega \mathcal{R} \theta^{k} a}^{k ; j}= \begin{cases}-2\left(m_{a}^{j}+\frac{1}{2} n_{a}^{j}\right), & k=0 \\ 2^{1-s} n_{a}^{j}, & k=1\end{cases}
$$

for a $\mathbf{Z}_{2}$ orientifold with $(s=1)$ or without $(s=0)$ tilted tori $T_{2}^{j}$. In that case the two $O 6$-planes in $T_{2}^{j}$ are given by $\left(n_{O 6_{0}}^{j}, m_{O 6_{0}}^{j}\right)=(2,0)$ and $\left(n_{O 6_{1}}^{j}, m_{O 6_{1}}^{j}\right)=\left(0,2^{1-s}\right)$. Contributions $L_{i}$ from zero modes are possible, if brane $a$ and its image $\Omega \mathcal{R} \theta^{k} a$ are parallel w.r.t. some torus $i$. This is only possible, if brane $a$ sits on the orientifold plane $O 6_{k}$ w.r.t. that torus $T_{2}^{i}$, i.e. $v_{a}^{k ; i}=0$. In that case $\mathrm{KK}$ momenta parallel and windings orthogonal to the orientifold plane $O 6_{k}$ are invariant under the action $\Omega \mathcal{R} \theta^{k}$ and contribute to the sum

$$
L_{i}\left(t, T^{i}, V_{O 6_{k}}^{i}\right)=\sum_{r, s \in \mathbf{Z}} e^{-\frac{\pi t}{T_{2}^{i} V_{O 6_{k}}^{i}}\left|r+2^{\mu} T^{i} s\right|^{2}},
$$


with $\mu=0$ for $\mathbf{Z}_{2}, \mathbf{Z}_{4}$ orbifolds with $A$-type lattice and $\mu=1$ in the case of $B$-type lattice, and

$$
V_{O 6_{k}}^{i}=\left[\frac{R_{1}^{j}}{R_{2}^{j}} n_{a}^{j} n_{O 6_{k}}^{j}+\frac{R_{2}^{j}}{R_{1}^{j}} m_{a}^{j} m_{O 6_{k}}^{j}+\left(n_{a}^{j} m_{O 6_{k}}^{j}+n_{O 6_{k}}^{j} m_{a}^{j}\right) \cos \alpha^{j}\right] \frac{1}{\sin \alpha^{j}} .
$$

Except for the $\mathbf{Z}_{2}$ and $\mathbf{Z}_{4}$ orbifold with $A$-type lattice the windings are doubled compared to the winding contribution of the annulus diagram (2.19). We refer the reader to the original literature [12,13,26,27] concerning the possible choices of lattices for a given orbifold action. In addition, for orbifolds other than $\mathbf{Z}_{2}$ we have to choose $R_{1}^{i}=R_{2}^{i}$.

\subsection{Gauged open string partition functions in the closed string channel}

Finally, in this subsection we present the gauged partition functions, given in Eqs. (2.17), (2.21), and (2.23) in the closed string channel, i.e. replacing the modular parameter $t$ by $l=1 / t$ and $l=1 /(4 t)$ for the annulus and Möbius amplitude, respectively. This yields the following expressions, which we shall need in the following sections

$$
\begin{aligned}
\widetilde{A}_{a b}^{d}(B) & =2^{d-3}(-1)^{d} l \beta_{a b} \prod_{i=1}^{d^{\prime}} V_{a}^{i} \widetilde{Z}_{i}\left(l, T^{i}, V_{a}^{i}\right) \\
& \times \sum_{\vec{\delta}} s \vec{\delta} \frac{\theta\left[\begin{array}{l}
\delta_{1} \\
\delta_{2}
\end{array}\right]\left(\epsilon_{a b}, 2 i l\right)}{\theta\left[\begin{array}{l}
\frac{1}{2} \\
\frac{1}{2}
\end{array}\right]\left(\epsilon_{a b}, 2 i l\right)} \frac{\theta\left[\begin{array}{l}
\delta_{1} \\
\delta_{2}
\end{array}\right](0,2 i l)^{3-d}}{\eta(2 i l)^{9-3 d}} \prod_{j=1}^{d} I_{a b}^{j} \frac{\theta\left[\begin{array}{l}
\delta_{1} \\
\delta_{2}
\end{array}\right]\left(i v_{a b}^{j}, 2 i l\right)}{\theta\left[\begin{array}{l}
\frac{1}{2} \\
\frac{1}{2}
\end{array}\right]\left(i v_{a b}^{j}, 2 i l\right)} \\
\widetilde{A}_{a a^{\prime}}^{d}(B) & =2^{d-3}(-1)^{d} l \beta_{a a^{\prime}} B \prod_{i=1}^{d^{\prime}} V_{a}^{i} \widetilde{Z}_{i}\left(l, T^{i}, V_{a}^{i}\right) \\
& \times \sum_{\vec{\delta}} s_{\vec{\delta}} \frac{\theta\left[\begin{array}{l}
\delta_{1} \\
\delta_{2}
\end{array}\right]\left(\epsilon_{a a^{\prime}}, 2 i l\right)}{\theta\left[\begin{array}{l}
\frac{1}{2} \\
\frac{1}{2}
\end{array}\right]\left(\epsilon_{a a^{\prime}}, 2 i l\right)} \frac{\theta\left[\begin{array}{l}
\delta_{1} \\
\delta_{2}
\end{array}\right](0,2 i l)^{3-d}}{\eta(2 i l)^{9-3 d}} \prod_{j=1}^{d} I_{a a^{\prime}}^{j} \frac{\theta\left[\begin{array}{l}
\delta_{1} \\
\delta_{2}
\end{array}\right]\left(i v_{a a^{\prime}}^{j}, 2 i l\right)}{\theta\left[\begin{array}{l}
\frac{1}{2} \\
\frac{1}{2}
\end{array}\right]\left(i v_{a a^{\prime}}^{j}, 2 i l\right)}
\end{aligned}
$$

with the resummed lattice function:

$$
\widetilde{Z}_{i}\left(l, T^{i}, V_{a}^{i}\right)=\sum_{r, s \in \mathbf{Z}} e^{-\pi l \frac{V_{a}^{i}}{T_{2}^{i}}\left|r+T^{i} s\right|^{2}} .
$$

Furthermore for (2.23) we obtain:

$$
\begin{aligned}
\widetilde{M}_{a, \Omega \mathcal{R} \theta^{k} a}^{k ; d}(B) & =-2^{5-d}(-1)^{d} l \beta_{a} \prod_{i=1}^{d^{\prime}} n_{O 6_{k}}^{i} V_{O 6_{k}}^{i} \widetilde{L}_{i}\left(4 l, T^{i}, V_{O 6_{k}}^{i}\right) \sum_{\vec{\delta}} s_{\vec{\delta}} \frac{\theta\left[\begin{array}{l}
\delta_{1} \\
\delta_{2}
\end{array}\right]\left(\frac{\epsilon_{a}}{2}, 2 i l-\frac{1}{2}\right)}{\theta\left[\begin{array}{l}
\frac{1}{2} \\
\frac{1}{2}
\end{array}\right]\left(\frac{\epsilon_{a}}{2}, 2 i l-\frac{1}{2}\right)} \\
& \times \prod_{i=1}^{d^{\prime}} \frac{\theta\left[\begin{array}{l}
\delta_{1} \\
\delta_{2}
\end{array}\right]\left(0,2 i l-\frac{1}{2}\right)^{3-d}}{\eta\left(2 i l-\frac{1}{2}\right)^{9-3 d}} \prod_{j=1}^{d} 2^{\delta_{j}} \frac{I_{a, \Omega \mathcal{R} \theta^{k} a}^{k ; j} \theta\left[\begin{array}{l}
\delta_{1} \\
\delta_{2}
\end{array}\right]\left(i v_{a}^{k ; j}, 2 i l-\frac{1}{2}\right)}{\theta\left[\begin{array}{c}
\frac{1}{2} \\
\frac{1}{2}
\end{array}\right]\left(i v_{a}^{k ; j}, 2 i l-\frac{1}{2}\right)}
\end{aligned}
$$


with the Poisson resummed expression

$$
\widetilde{L}_{i}\left(l, T^{i}, V_{O 6_{k}}^{i}\right)=\frac{1}{2^{\mu}} \sum_{r, s \in \mathbf{Z}} e^{-\pi l \frac{V_{O 6_{k}}^{i}}{T_{2}^{i}}\left|2^{-\mu} r+T^{i} s\right|^{2}},
$$

with $\mu=0$ for $\mathbf{Z}_{2}, \mathbf{Z}_{4}$ orbifolds with $A$-type lattice and $\mu=1$ otherwise.

\section{One-loop gauge corrections in supersymmetric intersecting brane world models}

In the following we consider $K$ stacks $a, b, \ldots$ of branes in an $\mathrm{N}=1$ orientifold/orbifold background. Each stack $a$ has $N_{a}$ parallel branes constituing the gauge group $G_{a}$ after the orbifold/orientifold group action. To handle the various mirror images of a given stack under the orientifold/orbifold actions it proved to be convenient to introduce the notation of a conjugacy class $[a]$ associated to each stack $a$. Of course, each member of the conjugacy class $[a]$ appears itself to be a stack of $N_{a}$ copies of the same branes. ( $c f$. the previous section for further details).

\subsection{Contributions to the one-loop gauge corrections}

To obtain the gauge-threshold corrections w.r.t. a given gauge group $G_{a}$ one has to extract the order $\mathcal{O}\left(B^{2}\right)$ part from the gauged partition functions (2.17), (2.21) and (2.23). A system of intersecting branes may give rise to various sectors with different space-time supersymmetries. The amount of supercharges which is preserved by a pair of distinct stacks $a$ and $b$ (or two stacks $a, a^{\prime}$ from the same conjugacy class $[a]$ ) depends on their relative angles $\phi_{a b}^{j}\left(\phi_{a a^{\prime}}^{j}\right)$ w.r.t. the internal complex dimensions, given in $E q$. (2.18). Open string exchanges between parallel branes (i.e. $I_{a b}=0$ or $I_{a a^{\prime}}=0$ ) preserve sixteen supercharges and therefore do not lead to any gauge coupling renormalization. On the other hand open string exchanges from sectors, which preserve either $\mathrm{N}=1$ or $\mathrm{N}=2$ supersymmetry, give rise to an non-vanishing one-loop gauge correction. Hence, if one $\phi_{a b}^{j} \neq 0$ (or $\phi_{a a^{\prime}}^{j} \neq 0$ ) in one torus $T_{2}^{j}$, a portion of the sixteen supersymmetries is broken and we expect non-vanishing one-loop gauge couplings for these cases.

According to the discussion in subsection 2.2 several origins for non-vanishing oneloop gauge corrections may be possible: The first case, where stack $a \in[a]$ associated to the gauge group $G_{a}$ under consideration, preserves $\mathrm{N}=1$ or $\mathrm{N}=2$ supersymmetry with a stack $b \in[b]$ from a different stack $b$. These cases, collectively denoted as $a b$-exchange, are described by the gauged annulus partition functions $A_{a b}^{d=3,2}(B)$, presented in (2.17). They give rise to the one-loop gauge corrections $\Delta_{a b}^{N=1,2}$, respectively. Second, $\mathrm{N}=1$ or $\mathrm{N}=2$ supersymmetric sectors are possible for open string exchanges between two stacks 
$a, a^{\prime} \in[a]$ stemming from the same conjugacy class. These diagrams, summarized as $a a^{\prime}$-exchange and described by the amplitude $A_{a a^{\prime}}^{d=3,2}(B)$, lead to the corrections $\Delta_{a a^{\prime}}^{N=1,2}$. Finally, depending on the amount of supersymmetry preserved by the two stacks $a$ and $\Omega \mathcal{R} \theta^{k} a$, the gauged Möbius diagram $M_{a, \Omega \mathcal{R} \theta^{k} a}^{k}(B)$ gives rise to the one-loop gauge corrections $8 \Delta_{a, \Omega \mathcal{R} \theta^{k} a}^{k ; N=1}$ and $\Delta_{a, \Omega \mathcal{R} \theta^{k} a}^{k ; N=2}$, respectively. The latter describe open strings starting on brane $a$ and ending on its orientifold image $\Omega \mathcal{R} \theta^{k} a$.

To summarize, let us present the complete one-loop correction to the gauge coupling $g_{a}^{-2}$ of the gauge group $G_{a}$

$$
\Delta_{G_{a}}=\sum_{\substack{b=1 \\ b \neq a}}^{K} \sum_{\substack{a \in[a] \\ b \in[b]}} \Delta_{a b}+\sum_{\substack{a, a^{\prime} \in[a] \\ a \neq a^{\prime}}} \Delta_{a a^{\prime}}+\sum_{a \in[a]} \sum_{k=0}^{N-1} \Delta_{a, \Omega \mathcal{R} \theta^{k} a}^{k}
$$

with the Schwinger integrals (converted into the closed string channel):

$$
\begin{gathered}
\Delta_{a b}^{N=1,2}=\left.2 \pi^{-2} \int_{0}^{\infty} \frac{d l}{l} \operatorname{Tr} \frac{\partial^{2}}{\partial B^{2}} \widetilde{A}_{a b}^{d=3,2}(B)\right|_{B=0}, \\
\Delta_{a a^{\prime}}^{N=1,2}=\left.2 \pi^{-2} \int_{0}^{\infty} \frac{d l}{l} \operatorname{Tr} \frac{\partial^{2}}{\partial B^{2}} \widetilde{A}_{a a^{\prime}}^{d=3,2}(B)\right|_{B=0} \\
\Delta_{a, \Omega \mathcal{R} \theta^{k} a}^{k ; N=1,2}=\left.\pi^{-2} \int_{0}^{\infty} \frac{d l}{l} \operatorname{Tr} \frac{\partial^{2}}{\partial B^{2}} \widetilde{M}_{a, \Omega \mathcal{R} \theta^{k} a}^{k ; d=3,2}(B)\right|_{B=0} .
\end{gathered}
$$

The factor 2 in the front of the interals accounts for the two different orientations of the open strings w.r.t. to stack $a$. The group trace Tr is accomplished by summing over all string endpoint charges $q_{a}, q_{b}$ ( $q_{a^{\prime}}$, respectively) augmented with the orientifold projection 9 . We will be more precise about that in the following subsections. In addition, on the symbol $\Delta$ we have put the subscript $N=1,2$ referring to the amount of supersymmetries preserved by the two branes involved. As described before, $\mathrm{N}=1,2$ supersymmetries are respectively related to $d=3,2$ tori $T_{2}^{j}$, in which the branes have non-vanishing intersections. The sum (3.1) represents a sum over various open string sectors in close analogy to the expressions (2.8) and (2.10). However, the sum (3.1) includes much fewer sectors, since at least one

8 In the case of a Möbius diagram we shall always keep the twist order $k$ as subscript on the correction $\Delta_{a, \Omega \mathcal{R} \theta^{k} a}^{k ; N=1,2}$ in order to distungish it from the correction from an annulus diagram $\Delta_{a a^{\prime}}^{N=1,2}$.

9 The phase $\rho_{\Omega \mathcal{R} \theta^{k}}$ in the sum (2.10), which arises from (2.11), will show up again after performing the traces within the intergal $\Delta_{a, \Omega \mathcal{R} \theta^{k} a}^{k ; N=1,2}$. 
open string end has to couple to a brane of gauge group $G_{a}$. In addition $\mathrm{N}=4$ sectors do not contribute.

After these preparations let us now determine the general form of the one-loop gauge threshold correction (3.1) by evaluating the Schwinger integrals (3.2) for $\mathrm{N}=1$ and $\mathrm{N}=2$ supersymmetric sectors of intersecting brane world models, leading to the results for $\Delta_{a b}^{\mathrm{N}=1,2}, \Delta_{a a^{\prime}}^{\mathrm{N}=1,2}$ and $\Delta_{a, \Omega \mathcal{R} \theta^{k} a}^{k, \mathrm{~N}=1,2}$, respectively. This will be accomplished in several steps: From the closed string expressions (2.29), (2.30), and (2.32) entering (3.2) we determine that piece which is second order in the magnetic field $B$, perform the spin-structure sum and eventually do the integration. In additon we must clarify the possible existence of $U V$ divergences in (3.1). We give the general rules for vanishing $U V$-divergences in subsection 3.4. In subsection 3.7 we apply our results to present the gauge thresholds for a $\mathbf{Z}_{2} \times \mathbf{Z}_{2}$ orientifold model.

\subsection{Gauge thresholds from $N=1$ supersymmetric sectors}

Let us first determine $\Delta_{a b}^{\mathrm{N}=1}$ for the case when stack $a$ and $b$ preserve $\mathrm{N}=1$ supersymmetry. To extract from $\widetilde{A}_{a b}(B)$ the second order in the magnetic field $B$ we use

$$
\begin{aligned}
\pi q_{a} & \left.\frac{\partial^{2}}{\partial B^{2}} B \frac{\theta\left[\begin{array}{l}
\delta_{1} \\
\delta_{2}
\end{array}\right]\left(\epsilon_{a b}, \tau\right)}{\theta\left[\begin{array}{l}
\frac{1}{2} \\
\frac{1}{2}
\end{array}\right]\left(\epsilon_{a b}, \tau\right)}\right|_{B=0} \\
& =-\frac{\pi^{2} q_{a}^{2}}{\eta^{3}}\left\{\frac{1}{3} \theta\left[\begin{array}{l}
\delta_{1} \\
\delta_{2}
\end{array}\right](0, \tau)+\frac{1}{6} E_{2}(\tau) \theta\left[\begin{array}{l}
\delta_{1} \\
\delta_{2}
\end{array}\right](0, \tau)+\frac{1}{2 \pi^{2}} \theta^{\prime \prime}\left[\begin{array}{l}
\delta_{1} \\
\delta_{2}
\end{array}\right](0, \tau)\right\},
\end{aligned}
$$

with $e^{2 \pi i \epsilon_{a b}}=\frac{1+i \pi q_{a} B}{1-i \pi q_{a} B}$, given in $E q$. (2.15). For the $\mathrm{N}=1$ supersymmetric sector only the last term of (3.3) gives rise to a non-vanishing contribution to (3.1) after performing the spin-structure sum. That contribution we have calculated in the Eq. (B.2). Hence the gauge threshold corrections for an $\mathrm{N}=1$ sector take 10 the form

$$
\Delta_{a b}^{\mathrm{N}=1}=-2 \pi^{-1} b_{a b}^{N=1} \int_{0}^{\infty} d l\left[\frac{\theta_{1}^{\prime}\left(i v_{a b}^{1}, 2 i l\right)}{\theta_{1}\left(i v_{a b}^{1}, 2 i l\right)}+\frac{\theta_{1}^{\prime}\left(i v_{a b}^{2}, 2 i l\right)}{\theta_{1}\left(i v_{a b}^{2}, 2 i l\right)}+\frac{\theta_{1}^{\prime}\left(i v_{a b}^{3}, 2 i l\right)}{\theta_{1}\left(i v_{a b}^{3}, 2 i l\right)}\right]
$$

for the case $v_{a b}^{1}+v_{a b}^{2}+v_{a b}^{3}=0$. Here the $\mathrm{N}=1 \beta$-function coefficient is given by:

$$
b_{a b}^{N=1}=I_{a b} \operatorname{Tr}\left(Q_{a}^{2} \gamma_{1}^{a} \otimes \gamma_{1}^{b}\right)
$$

We reinstated the charge operator $Q_{a}$ of the gauge group $G_{a}$ and the matrices $\gamma_{1}^{a}, \gamma_{1}^{b}$ acting on the Chan-Paton states at the endpoints of the open strings. They fulfill $\gamma_{1}^{b}=\mathbf{1}_{N_{b}}$

10 In the following, from the possible realizations of $\mathrm{N}=1$ supersymmetry, $\pm v_{a b}^{1} \pm v_{a b}^{2} \pm v_{a b}^{3}=0$, we investigate the case $v_{a b}^{1}+v_{a b}^{2}+v_{a b}^{3}=0$. The other cases follow from the latter by changing signs. 
112,13, since $N_{b}$ branes sit on top of each brane from stack $b$. The order of $Q_{a}$ and $\gamma_{1}^{a}$ in the trace is not relevant, since $Q_{a}$ commutes with $\gamma_{1}^{a}$. Otherwise the gauged open string state would have not survived the orientifold projection. With this information we obtain:

$$
b_{a b}^{N=1}=I_{a b} \operatorname{Tr}\left(Q_{a}^{2} \gamma_{1}^{a}\right) \operatorname{Tr}\left(\gamma_{1}^{b}\right)=N_{b} I_{a b} \operatorname{Tr}\left(Q_{a}^{2}\right) .
$$

At this point, we easily see why annulus diagrams $A_{a b}$ with $\mathbf{Z}_{2}$ twist insertions do not give rise to one-loop gauge corrections. Due to (2.7), i.e. $\operatorname{Tr} \gamma_{\theta^{N / 2}}^{a}=0$, following from the cancellation of twisted sector tadpole contributions, annulus diagrams with $\mathbf{Z}_{2}$ twist insertions give rise to vanishing $\beta$-function coefficients, i.e. $\Delta_{a b}=0$. Finally, we have defined: $I_{a b}=\prod_{j=1}^{3} I_{a b}^{j}$.

The integrand of (3.4) does not change when $i v_{a b}^{j}$ is shifted into the region $-\pi<$ $i v_{a b}^{j}<\pi$ by integers of \pm 1 . Hence we may perform shifts on the angles $\phi_{b a}^{j}$ such that eventually $\left|i v_{a b}\right|<\pi$ is achieved. However only those shifts are allowed, which preserve the condition $v_{a b}^{1}+v_{a b}^{2}+v_{a b}^{3}=0$. To evaluate (3.4) we start from the relation

$$
\frac{\theta_{1}^{\prime}(i v, \tau)}{\theta_{1}(i v, \tau)}=-i \frac{\partial}{\partial v} \ln \theta_{1}(i v, \tau)=-\pi i \operatorname{coth}(\pi v)-\sum_{k=1}^{\infty} \alpha_{2 k}(i v)^{2 k-1}\left[E_{2 k}(\tau)-1\right]
$$

which can be derived from the identities [28]:

$$
\frac{z \theta_{1}^{\prime}(0, \tau)}{\theta_{1}(z, \tau)}=e^{\sum_{k=1}^{\infty} \frac{\alpha_{2 k}}{2 k} z^{2 k} E_{2 k}(\tau)} \quad, \quad \alpha_{2 k}=-\frac{(2 \pi i)^{2 k}}{(2 k) !} B_{2 k}=2 \zeta(2 k)
$$

and:

$$
\sum_{k=1}^{\infty} \frac{\alpha_{2 k}}{2 k} z^{2 k}=\ln (\pi z)-\ln \sin (\pi z) \quad, \quad 0<z<1 .
$$

After inserting (3.7) into (3.4), the first term of (3.7) gives rise to the following apparent divergent 11 integral:

$$
\delta_{a b}^{\mathrm{N}=1}=2 i b_{a b}^{N=1}\left[\operatorname{coth}\left(\pi v_{a b}^{1}\right)+\operatorname{coth}\left(\pi v_{a b}^{2}\right)+\operatorname{coth}\left(\pi v_{a b}^{3}\right)\right] \int_{0}^{\infty} d l,
$$

with (cf. Eq. (C.3)):

$$
\operatorname{coth}\left(\pi v_{a b}^{j}\right)=i \cot \left(\phi_{b}^{j}-\phi_{a}^{j}\right)=i \frac{n_{a}^{j} n_{b}^{j} \frac{R_{1}^{j}}{R_{2}^{j}}+m_{a}^{j} m_{b}^{j} \frac{R_{2}^{j}}{R_{1}^{j}}+\left(n_{a}^{j} m_{b}^{j}+n_{b}^{j} m_{a}^{j}\right) \cos \alpha^{j}}{n_{a}^{j} m_{b}^{j}-n_{b}^{j} m_{a}^{j}} \frac{1}{\sin \alpha^{j}} .
$$

${ }^{11} U V$ divergent in the open string sector. 
The expression (3.10) can be identified with the $U V$ divergence stemming from the $N S$ sector, derived in Eq. (C.11) (cf. subsection 3.4). Note, the additional factor of 2 in (3.10) from the two different open string orientations. It will prove to be important for the cancellation of $N S$ tadpole contributions in the complete expression (3.1), after taking into account similar divergences from the other sectors. We shall discuss them later and discard $\delta_{a b}^{\mathrm{N}=1}$ for a short moment to come back to it in subsection 3.4.

Let us now proceed with the sum of (3.7), which represents the pure string contribution and gives rise to the following type of integrals:

$$
\int_{0}^{\infty} d y\left[E_{2 n}(i y)-1\right] .
$$

A naive integration of the Eisenstein function $E_{2 n}(i y)$, given as power series in $e^{-2 \pi y}$, would lead to a non-converging series. The situation is to be contrasted with integrals over the torus fundmantal region of modular invariant functions as they arise from closed string one-loop amplitudes [29]. In these case, the $U V$ region $y \rightarrow 0$ is excluded, which allows to use an expansion of the modular function w.r.t to $e^{-2 \pi y}$ and leads to finite results. On more mathematical grounds, the integral (3.12) represents an isomorphism between a modular form and a Dirichlet series. This problem has been studied by Hecke and we will apply his methods in Appendix A. In fact, there we shall see, that the integrals (3.12) can be evaluated after analytic continuation with the result12 (A.16):

$$
\begin{aligned}
& \int_{0}^{\infty} d l\left[E_{2 n}(2 i l)-1\right]=\frac{2^{-2 n} \pi^{1-2 n}(2 n) !}{(1-2 n)\left|B_{2 n}\right|} \zeta(2 n-1), n>1 \\
& \int_{0}^{\infty} d l l^{\epsilon}\left[E_{2}(2 i l)-1\right]=\frac{3}{\pi \epsilon}-\frac{3}{\pi} \ln 2 .
\end{aligned}
$$

Essentially the parameter $\epsilon>0$ originates from a dimensional regularization (cf. also [30]). The term $\frac{3}{\pi \epsilon}-\frac{3}{\pi} \ln 2$ accounts for the infrared divergence of massless closed string modes. The summand $k=1$ of (3.7) is linear in $i v$, which thanks to supersymmetry, vanishes in the expression (3.4) after summing over all three complex planes. Hence we may drop the case $k=1$. With the results (3.13) the last sum of (3.7) can be integrated to:

$$
\begin{aligned}
\int_{0}^{\infty} d l \sum_{k=2}^{\infty} \alpha_{2 k}(i v)^{2 k-1}\left[E_{2 k}(2 i l)-1\right] & =-\pi \sum_{k=2}^{\infty}(i v)^{2 k-1} \frac{\zeta(2 k-1)}{2 k-1} \\
& =-\frac{1}{2} \pi \ln \left[e^{-2 i \gamma_{E} v} \frac{\Gamma(1-i v)}{\Gamma(1+i v)}\right] .
\end{aligned}
$$

12 We use $\zeta(2 n)=\frac{1}{(2 n) !} 2^{2 n}-1 \pi^{2 n}\left|B_{2 n}\right|$. 
For the last step we used the identity [31]:

$$
\sum_{n=1}^{\infty} \frac{x^{2 n+1}}{2 n+1} \zeta(2 n+1)=\frac{1}{2} \ln \left[e^{-2 \gamma_{E} x} \frac{\Gamma(1-x)}{\Gamma(1+x)}\right] \quad, \quad x \in \mathbf{R},|x|<1 .
$$

After these preparations we are ready to determine (3.4). For $\left|i v_{a b}^{j}\right|=\frac{1}{\pi}\left|\phi_{b a}^{j}\right|<1$ we obtain:

$$
\Delta_{a b}^{\mathrm{N}=1}=\delta_{a b}^{\mathrm{N}=1}-b_{a b}^{N=1} \ln \frac{\Gamma\left(1-\frac{1}{\pi} \phi_{b a}^{1}\right) \Gamma\left(1-\frac{1}{\pi} \phi_{b a}^{2}\right) \Gamma\left(1+\frac{1}{\pi} \phi_{b a}^{1}+\frac{1}{\pi} \phi_{b a}^{2}\right)}{\Gamma\left(1+\frac{1}{\pi} \phi_{b a}^{1}\right) \Gamma\left(1+\frac{1}{\pi} \phi_{b a}^{2}\right) \Gamma\left(1-\frac{1}{\pi} \phi_{b a}^{1}-\frac{1}{\pi} \phi_{b a}^{2}\right)} .
$$

Here the moduli dependence is given implicitly through the relation (3.11). In subsection 3.6 we shall present an alternative expression for (3.16) showing its explicit dependence on the radii $R_{1}^{i}, R_{2}^{i}$. Due to the symmetry behaviour of $b_{a b}^{N=1}$, following from $I_{a b} \leftrightarrow-I_{a b}$ under the exchange of the two branes $a \leftrightarrow b$, the whole result $\Delta_{a b}^{\mathrm{N}=1}$ does not alter under permuting the brane $a$ and $b$.

Let us now come to the Möbius sector (2.23), describing an open string starting from brane $a$ and ending on $a^{\prime}=\Omega \mathcal{R} \theta^{k} a$. Similar as before, using $E q$. (3.3) and the spinstructure sum (B.2), the correction from the $\mathrm{N}=1$ sector can be expressed by $\left(\tau=2 i l-\frac{1}{2}\right)$

$$
\Delta_{a, \Omega \mathcal{R} \theta^{k} a}^{k ; \mathrm{N}=1}=-4 \pi^{-1} b_{a, \Omega \mathcal{R} \theta^{k} a}^{k ; N=1} \int_{0}^{\infty} d l\left[\frac{\theta_{1}^{\prime}\left(i v_{a}^{k ; 1}, \tau\right)}{\theta_{1}\left(i v_{a}^{k ; 1}, \tau\right)}+\frac{\theta_{1}^{\prime}\left(i v_{a}^{k ; 2}, \tau\right)}{\theta_{1}\left(i v_{a}^{k ; 2}, \tau\right)}+\frac{\theta_{1}^{\prime}\left(i v_{a}^{k ; 3}, \tau\right)}{\theta_{1}\left(i v_{a}^{k ; 3}, \tau\right)}\right]
$$

for the case $v_{a}^{k ; 1}+v_{a}^{k ; 2}+v_{a}^{k ; 3}=0$, with the $\beta$-function coefficient:

$$
b_{a, \Omega \mathcal{R} \theta^{k} a}^{k ; \mathrm{N}=1}=-2 I_{a, \Omega \mathcal{R} \theta^{k} a}^{k} \operatorname{Tr}\left[Q_{a}^{2}\left(\gamma_{\Omega \mathcal{R} \theta^{k}}^{\Omega \mathcal{R} \theta^{k} a}\right)^{*} \gamma_{\Omega \mathcal{R} \theta^{k}}^{a}\right]=-2 N_{a} \rho_{\Omega \mathcal{R} \theta^{k}} I_{a ; O 6_{k}} \operatorname{Tr}\left(Q_{a}^{2}\right) .
$$

We used (2.11) to perform the trace over the $\gamma$-matrices. As before for $\Delta_{a b}^{\mathrm{N}=1}$, with the help of (3.7), we may disentangle $U V$-divergent contribution of (3.17):

$$
\delta_{a, \Omega \mathcal{R} \theta^{k} a}^{k ; \mathrm{N}=1}=4 i b_{a}^{k ; N=1}\left[\operatorname{coth}\left(\pi v_{a}^{k ; 1}\right)+\operatorname{coth}\left(\pi v_{a}^{k ; 2}\right)+\operatorname{coth}\left(\pi v_{a}^{k ; 3}\right)\right] \int_{0}^{\infty} d l .
$$

In addition, we have defined $I_{a ; O 6_{k}}=\prod_{j=1}^{3} I_{a ; O 6_{k}}^{j}$. With the integrals

$$
\begin{aligned}
& \int_{0}^{\infty} d l\left[E_{2 n}\left(2 i l-\frac{1}{2}\right)-1\right]=2^{2 n-4} \frac{2^{-2 n} \pi^{1-2 n}(2 n) !}{(1-2 n)\left|B_{2 n}\right|} \zeta(2 n-1), n>1 \\
& \int_{0}^{\infty} d l l^{\epsilon}\left[E_{2}\left(2 i l-\frac{1}{2}\right)-1\right]=\frac{3}{4 \pi \epsilon}+\frac{3}{4 \pi} \ln 2
\end{aligned}
$$


following from (A.17) the finite part of (3.17) can be integrated to give a similar expression than (3.16)

$$
\Delta_{a, \Omega \mathcal{R} \theta^{k} a}^{k ; \mathrm{N}=1}=\delta_{a, \Omega \mathcal{R} \theta^{k} a}^{k ; \mathrm{N}=1}-\frac{1}{4} b_{a, \Omega \mathcal{R} \theta^{k} a}^{k ; \mathrm{N}=1} \ln \frac{\Gamma\left(1-\frac{2}{\pi} \phi_{a}^{k ; 1}\right) \Gamma\left(1-\frac{2}{\pi} \phi_{a}^{k ; 2}\right) \Gamma\left(1+\frac{2}{\pi} \phi_{a}^{k ; 1}+\frac{2}{\pi} \phi_{a}^{k ; 2}\right)}{\Gamma\left(1+\frac{2}{\pi} \phi_{a}^{k ; 1}\right) \Gamma\left(1+\frac{2}{\pi} \phi_{a}^{k ; 2}\right) \Gamma\left(1-\frac{2}{\pi} \phi_{a}^{k ; 1}-\frac{1}{\pi} \phi_{a}^{k ; 2}\right)}
$$

with the hidden moduli dependence encoded in the angles (2.24) through through:

$$
\begin{aligned}
\operatorname{coth}\left(\pi v_{a}^{k ; j}\right) & =i \cot \left(\phi_{a}^{k ; j}\right)=i \cot \left(\phi_{a}^{j}-\phi_{O 6_{k}}^{j}\right) \\
& =\frac{i}{I_{O 6_{k}, a}^{j}}\left[n_{O 6_{k}}^{j} n_{a}^{j} \frac{R_{1}^{j}}{R_{2}^{j}}+m_{O 6_{k}}^{j} m_{a}^{j} \frac{R_{2}^{j}}{R_{1}^{j}}+\left(n_{O 6_{k}}^{j} m_{a}^{j}+n_{a}^{j} m_{O 6_{k}}^{j}\right) \cos \alpha^{j}\right] \frac{1}{\sin \alpha^{j}} .
\end{aligned}
$$

Again, the angles $\phi_{a}^{k ; j}$ have to be shifted into the domain $-\frac{\pi}{2}<\phi_{a}^{k ; j}<\frac{\pi}{2}$ by integers of $\pm \pi$. However only those shifts are allowed, which do not alter the integrand of (3.17) and obey the supersymmetry condition $\phi_{a}^{k ; 1}+\phi_{a}^{k ; 2}+\phi_{a}^{k ; 3}=0$.

Finally, let us move on to the corrections $\Delta_{a a^{\prime}}$ arsing from an open string exchange between a stack $a$ and one of its mirrors $a^{\prime} \in[a]$. We first note the identity

$$
\begin{aligned}
\pi\left(q_{a}+q_{a^{\prime}}\right) & \left.\frac{\partial^{2}}{\partial B^{2}} B \frac{\theta\left[\begin{array}{l}
\delta_{1} \\
\delta_{2}
\end{array}\right]\left(\epsilon_{a a^{\prime}}, \tau\right)}{\theta\left[\begin{array}{l}
\frac{1}{2} \\
\frac{1}{2}
\end{array}\right]\left(\epsilon_{a a^{\prime}}, \tau\right)}\right|_{B=0} \\
& =-\pi^{2} \frac{q_{a}^{2}+q_{a^{\prime}}^{2}}{\eta^{3}}\left\{\frac{1}{3} \theta\left[\begin{array}{l}
\delta_{1} \\
\delta_{2}
\end{array}\right](0, \tau)+\frac{1}{6} E_{2}(\tau) \theta\left[\begin{array}{l}
\delta_{1} \\
\delta_{2}
\end{array}\right](0, \tau)+\frac{1}{2 \pi^{2}} \theta^{\prime \prime}\left[\begin{array}{l}
\delta_{1} \\
\delta_{2}
\end{array}\right](0, \tau)\right\} \\
& +\pi^{2} \frac{q_{a} q_{a^{\prime}}}{\eta^{3}}\left\{\frac{1}{3} \theta\left[\begin{array}{l}
\delta_{1} \\
\delta_{2}
\end{array}\right](0, \tau)-\frac{1}{3} E_{2}(\tau) \theta\left[\begin{array}{l}
\delta_{1} \\
\delta_{2}
\end{array}\right](0, \tau)-\frac{1}{\pi^{2}} \theta^{\prime \prime}\left[\begin{array}{l}
\delta_{1} \\
\delta_{2}
\end{array}\right](0, \tau)\right\}
\end{aligned}
$$

with $e^{2 \pi i \epsilon_{a a^{\prime}}}=\frac{\left(1+i \pi q_{a} B\right)\left(1+i \pi q_{a^{\prime}} B\right)}{\left(1-i \pi q_{a} B\right)\left(1-i \pi q_{a^{\prime}} B\right)}$, from $E q$. 2.15). This relation allows us to extract the $\mathcal{O}\left(B^{2}\right)$ part from $\widetilde{A}_{a a^{\prime}}(B)$. We do not discuss any further the second term of (3.23), since its factor in front has a charge combination, which corresponds to the trace: $\operatorname{Tr}\left(Q_{a} \gamma_{1}^{a}\right) \operatorname{Tr}\left(Q_{a^{\prime}} \gamma_{1}^{a^{\prime}}\right)=0$. These traces vanish

$$
\operatorname{Tr}\left(Q_{a} \gamma_{1}^{a}\right)=\operatorname{Tr}\left(Q_{a}\right)=0
$$

under the assumption, that the gauge group $G_{a}$ under consideration is not one of the possible anomalous $U(1)$ gauge groups of the theory under consideration. Putting the first piece of (3.23) into the spin-structure sum ( $c f$. appendix B.1.) yields $(\tau=2 i l)$ :

$$
\Delta_{a a^{\prime}}^{\mathrm{N}=1}=-2 \pi^{-1} b_{a a^{\prime}}^{N=1} \int_{0}^{\infty} d l\left[\frac{\theta_{1}^{\prime}\left(i v_{a a^{\prime}}^{1}, \tau\right)}{\theta_{1}\left(i v_{a a^{\prime}}^{1}, \tau\right)}+\frac{\theta_{1}^{\prime}\left(i v_{a a^{\prime}}^{2}, \tau\right)}{\theta_{1}\left(i v_{a a^{\prime}}^{2}, \tau\right)}+\frac{\theta_{1}^{\prime}\left(i v_{a a^{\prime}}^{3}, \tau\right)}{\theta_{1}\left(i v_{a a^{\prime}}^{3}, \tau\right)}\right] .
$$


with the $\beta$-coefficient

$$
b_{a a^{\prime}}^{N=1}=I_{a a^{\prime}}\left[\operatorname{Tr}\left(Q_{a}^{2} \gamma_{1}^{a}\right) \operatorname{Tr}\left(\gamma_{1}^{a^{\prime}}\right)+\operatorname{Tr}\left(\gamma_{1}^{a}\right) \operatorname{Tr}\left(Q_{a}^{2} \gamma_{1}^{a^{\prime}}\right)\right]=2 N_{a} I_{a a^{\prime}} \operatorname{Tr}\left(Q_{a}^{2}\right)
$$

The integral (3.25) contains an $U V$ divergence analogous to (3.10):

$$
\delta_{a a^{\prime}}^{\mathrm{N}=1}=2 i b_{a a^{\prime}}^{N=1}\left[\operatorname{coth}\left(\pi v_{a a^{\prime}}^{1}\right)+\operatorname{coth}\left(\pi v_{a a^{\prime}}^{2}\right)+\operatorname{coth}\left(\pi v_{a a^{\prime}}^{3}\right)\right] \int_{0}^{\infty} d l,
$$

which entirely steams from the $N S$-sector and will be further discussed in subsection 3.4. Like in the previous cases the integral (3.25) yields:

$$
\Delta_{a a^{\prime}}^{\mathrm{N}=1}=\delta_{a a^{\prime}}^{\mathrm{N}=1}-b_{a a^{\prime}}^{\mathrm{N}=1} \ln \frac{\Gamma\left(1-\frac{1}{\pi} \phi_{a^{\prime} a}^{1}\right) \Gamma\left(1-\frac{1}{\pi} \phi_{a^{\prime} a}^{2}\right) \Gamma\left(1+\frac{1}{\pi} \phi_{a^{\prime} a}^{1}+\frac{1}{\pi} \phi_{a^{\prime} a}^{2}\right)}{\Gamma\left(1+\frac{1}{\pi} \phi_{a^{\prime} a}^{1}\right) \Gamma\left(1+\frac{1}{\pi} \phi_{a^{\prime} a}^{2}\right) \Gamma\left(1-\frac{1}{\pi} \phi_{a^{\prime} a}^{1}-\frac{1}{\pi} \phi_{a^{\prime} a}^{2}\right)},
$$

In analogy to (3.11) the difference of the angles $\phi_{a}^{j}$ and $\phi_{a^{\prime}}^{j}$ are related to the radii through:

$$
\operatorname{coth}\left(\pi v_{a a^{\prime}}^{j}\right)=i \cot \left(\phi_{a^{\prime}}^{j}-\phi_{a}^{j}\right)=i \frac{n_{a}^{j} n_{a^{\prime}}^{j} \frac{R_{1}^{j}}{R_{2}^{j}}+m_{a}^{j} m_{a^{\prime}}^{j} \frac{R_{2}^{j}}{R_{1}^{j}}+\left(n_{a}^{j} m_{a^{\prime}}^{j}+n_{a^{\prime}}^{j} m_{a}^{j}\right) \cos \alpha^{j}}{n_{a}^{j} m_{a^{\prime}}^{j}-n_{a^{\prime}}^{j} m_{a}^{j}} \frac{1}{\sin \alpha^{j}} .
$$

\subsection{Gauge thresholds from $N=2$ supersymmetric sectors}

The discussion of $\mathrm{N}=2$ sectors is somewhat much simpler as for $\mathrm{N}=1$ sectors since branes of this sector represent $1 / 2$ BPS saturated objects and therefore only massless open string states contribute to the gauge coupling renormalization. This manifests in drastic simplifications in the gauged open string partition functions for this sector (at second order in the magnetic field $B$ ). For $\mathrm{N}=2$ sectors, whose spin-structure sum has been performed in appendix B.2., we have $v_{a b}^{i}=0$ w.r.t. the $i$-th plane and $v_{a b}^{j} \pm v_{a b}^{l}=0$ for the two remaining planes. With Eq. (B.3) the second integral of (3.2) boils down to:

$$
\Delta_{a b}^{\mathrm{N}=2}=b_{a b}^{\mathrm{N}=2} V_{a}^{i} \int_{0}^{\infty} d l \widetilde{Z}_{i}\left(l, T^{i}, V_{a}^{i}\right),
$$

with the $\mathrm{N}=2 \beta$-function coefficient:

$$
b_{a b}^{\mathrm{N}=2}=-2 I_{a b}^{j} I_{a b}^{l} \operatorname{Tr}\left(Q_{a}^{2} \gamma_{1}^{a}\right) \operatorname{Tr}\left(\gamma_{1}^{b}\right)=-2 N_{b} I_{a b}^{j} I_{a b}^{l} \operatorname{Tr}\left(Q_{a}^{2}\right) .
$$

Again as before the same argument about possible $\mathbf{Z}_{2}$ twist insertions applies: With (2.7) for such sectors the $\beta$-function coefficient and hence also $\Delta_{a b}^{\mathrm{N}=2}$ vanish. A divergence

$$
\delta_{a b}^{\mathrm{N}=2}=b_{a b}^{\mathrm{N}=2} V_{a}^{i} \int_{0}^{\infty} d l
$$


due to the zero momentum state $(r, s)=(0,0)$ in (2.31) is encountered in (3.30) for the limit $l \rightarrow \infty$. After inspection of (C.10) it is identified as a potential tadpole contribution in the $N S$-sector. As before for $\delta_{a b}^{\mathrm{N}=1}$, we shall discuss its relevance in subsection 3.4, where we shall conclude that in the complete expression (3.1) all $U V$-divergences are cancelled. Hence we split that term from the integral $\Delta_{a b}^{\mathrm{N}=2}$ and write

$$
\begin{aligned}
\Delta_{a b}^{\mathrm{N}=2} & =\delta_{a b}^{\mathrm{N}=2}+b_{a b}^{\mathrm{N}=2} V_{a}^{i} \int_{0}^{\infty} d l \sum_{(r, s) \neq(0,0)} e^{-\pi l \frac{V_{a}^{i}}{T_{2}^{i}}\left|r+T^{k} s\right|^{2}} \\
& =\delta_{a b}^{\mathrm{N}=2}-b_{a b}^{N=2}\left[\ln T_{2}^{i}\left|\eta\left(T^{i}\right)\right|^{4}+\ln V_{a}^{i}-\kappa\right]
\end{aligned}
$$

with the constant $\kappa=\gamma_{E}-\ln (4 \pi)$. The last integral has been already performed in Refs. [5]. Its $I R$-regularization is achieved by some sort of dimensional regularization [30]. We conclude, that apart from its second term $\ln V_{a}^{i}$ the functional form of the gauge threshold correction $\Delta_{a b}^{\mathrm{N}=2}$ is the same as we know already from $\mathrm{N}=2$ type $I$ orientifold compactifications [18,19].

Not much changes for the open string exchange between stack $a$ and one of its mirrors $a^{\prime}$. For this case we obtain the one-loop gauge correction

$$
\begin{aligned}
\Delta_{a a^{\prime}}^{\mathrm{N}=2} & =b_{a a^{\prime}}^{\mathrm{N}=2} V_{a}^{i} \int_{0}^{\infty} d l \widetilde{Z}_{i}\left(l, T^{i}, V_{a}^{i}\right) \\
& =\delta_{a a^{\prime}}^{\mathrm{N}=2}-b_{a a^{\prime}}^{N=2}\left[\ln T_{2}^{i}\left|\eta\left(T^{i}\right)\right|^{4}+\ln V_{a}^{i}-\kappa\right]
\end{aligned}
$$

with the $\mathrm{N}=2 \beta$-function coefficient ( $c f$. the arguments leading to $E q$. (3.26)):

$$
b_{a a^{\prime}}^{\mathrm{N}=2}=-2 I_{a a^{\prime}}^{j} I_{a a^{\prime}}^{l}\left[\operatorname{Tr}\left(Q_{a}^{2} \gamma_{1}^{a}\right) \operatorname{Tr}\left(\gamma_{1}^{a^{\prime}}\right)+\operatorname{Tr}\left(\gamma_{1}^{a}\right) \operatorname{Tr}\left(Q_{a}^{2} \gamma_{1}^{a^{\prime}}\right)\right]=-4 N_{a} I_{a a^{\prime}}^{j} I_{a a^{\prime}}^{l} \operatorname{Tr}\left(Q_{a}^{2}\right) .
$$

Again, in the $N S$ sector we have to face a divergence

$$
\delta_{a a^{\prime}}^{N=2}=b_{a a^{\prime}}^{\mathrm{N}=2} V_{a}^{i} \int_{0}^{\infty} d l
$$

from the zero momentum state $(r, s)=(0,0)$, to comment on later.

Finally let us come to the Möbius sector $M_{a ; \Omega \mathcal{R} \theta^{k} a}^{k}$ with $\mathrm{N}=2$ supersymmetry, i.e. brane $a$ and its image $\Omega \mathcal{R} \theta^{k} a$ are parallel within one torus $T_{2}^{i}$, i.e. $\phi_{a}^{k ; i}=0$. The insertion $\Omega \mathcal{R} \theta_{i}^{k}$ leaves invariant momenta parallel and windings orthogonal to the orientifold plane $O 6_{k}$ w.r.t. to the $i$-th plane. These states are encoded in the lattice sum $L_{i}\left(t, T^{i}, V_{O 6_{k}}^{i}\right)$, given in (2.27). In that case the relevant gauged partition function is (2.32), with $d^{\prime}=$ $1, d=2$. Its second order in $B$ may be obtained from $E q$. (B.3). It gives rise to the one-loop gauge correction

$$
\begin{aligned}
\Delta_{a ; \Omega \mathcal{R} \theta^{k} a}^{k ; \mathrm{N}=2} & =4 b_{a a^{\prime}}^{k ; N=2} V_{O 6_{k}}^{i} \int_{0}^{\infty} d l \widetilde{L}_{i}\left(4 l, T^{i}, V_{O 6_{k}}^{i}\right) \\
& =\delta_{a a^{\prime}}^{k ; \mathrm{N}=2}-b_{a a^{\prime}}^{k ; N=2}\left[\ln T_{2}^{i}\left|\eta\left(2^{\mu} T^{i}\right)\right|^{4}+\ln V_{O 6_{k}}^{i}+\ln 4-\kappa\right]
\end{aligned}
$$


with the $\mathrm{N}=2 \beta$-function coefficient ( $c f$. (2.11) ) :

$$
\begin{aligned}
b_{a a^{\prime}}^{k ; N=2} & =8 I_{O 6_{k} ; a}^{j} I_{O 6_{k} ; a}^{l} \operatorname{Tr}\left[Q_{a}^{2}\left(\gamma_{\Omega \mathcal{R}}^{\Omega \mathcal{R} a}\right)^{*} \gamma_{\Omega \mathcal{R}}^{a}\right] \\
& =8 N_{a} \rho_{\Omega \mathcal{R} \theta^{k}} I_{O 6_{k} ; a}^{j} I_{O 6_{k} ; a}^{l} \operatorname{Tr}\left(Q_{a}^{2}\right) .
\end{aligned}
$$

The potential tadpole contribution

$$
\delta_{a a^{\prime}}^{N=2}=2^{2-\mu} b_{a a^{\prime}}^{k ; N=2} V_{O 6_{k}}^{i} \int_{0}^{\infty} d l
$$

in the $N S$ sector from the zero momentum state $(r, s)=(0,0)$ will be discussed in a moment.

\subsection{Tadpole cancellation in one-loop gauge corrections}

One important question in any type $I$ one-loop calculation is the possible existence of $U V$ divergences of the integrals (3.2) in the open string channel $t \rightarrow 0$. For the one-loop vacuum amplitude, given by the sum of (2.8), (2.10) and the Klein bottle contribution, the cancellation of $U V$ divergences is guaranteed by the imposed tadpole cancellation. The question of absence of $U V$ divergences in the full expression (3.1) has to be addressed again. The background $B$, introduced in the open string partition function, may give rise to NS-tadpoles of the graviton, dilaton and two-index antisymmetric tensor through couplings in the Born-Infeld action. Furthermore, the R-piece of the $U V$ divergence may give rise to tadpoles of the Wess-Zumino type world brane couplings. These two types of divergences must vanish seperately in a consistent theory.

Eventually we would like all coefficients in front of the divergent integrals encountered before to add up to zero. Therefore, we investigate the coefficients of the integrals and define $\delta_{X}=\operatorname{Tr}\left(Q_{a}\right)^{2} \kappa_{X} \int_{0}^{\infty} d l$, with $X$ being one of the subscripts. Let us first collect the coefficients $\kappa$ of all potential divergent terms $\delta$ encountered in subsection 3.2 and refering to $\mathrm{N}=1$ supersymmetric sectors. We have 3.10

$$
\begin{aligned}
\kappa_{a b} & =2 i N_{b} I_{a b}\left[\operatorname{coth}\left(\pi v_{a b}^{1}\right)+\operatorname{coth}\left(\pi v_{a b}^{2}\right)+\operatorname{coth}\left(\pi v_{a b}^{3}\right)\right] \\
& =-2 N_{b} \sum_{\substack{(i, j, l)=\\
(1,2,3),(2,1,3),(3,1,2)}} \frac{1}{\sin \alpha^{i}}\left[n_{a}^{i} n_{b}^{i} \frac{R_{1}^{i}}{R_{2}^{i}}+m_{a}^{i} m_{b}^{i} \frac{R_{2}^{i}}{R_{1}^{i}}+\left(n_{a}^{i} m_{b}^{i}+n_{b}^{i} m_{a}^{i}\right) \cos \alpha^{i}\right] I_{a b}^{j} I_{a b}^{l} \\
& =-N_{b} \sum_{\substack{(i, j, l)=\\
(1,2,3),(2,1,3),(3,1,2)}} \frac{1}{U_{2}^{i}}\left[\left(n_{a}^{i}+m_{a}^{i} U^{i}\right)\left(n_{b}^{i}+m_{b}^{i} \bar{U}^{i}\right)+\left(n_{a}^{i}+m_{a}^{i} \bar{U}^{i}\right)\left(n_{b}^{i}+m_{b}^{i} U^{i}\right)\right] I_{a b}^{j} I_{a b}^{l},
\end{aligned}
$$


and a similar expression from (3.27):

$$
\begin{aligned}
\kappa_{a a^{\prime}} & =4 i N_{a} I_{a a^{\prime}}\left[\operatorname{coth}\left(\pi v_{a a^{\prime}}^{1}\right)+\operatorname{coth}\left(\pi v_{a a^{\prime}}^{2}\right)+\operatorname{coth}\left(\pi v_{a a^{\prime}}^{3}\right)\right] \\
& =-4 N_{a} \sum_{\substack{(i, j, l)=\\
(1,2,3),(2,1,3),(3,1,2)}} \frac{1}{\sin \alpha^{i}}\left[n_{a}^{i} n_{a^{\prime}}^{i} \frac{R_{1}^{i}}{R_{2}^{i}}+m_{a}^{i} m_{a^{\prime}}^{i} \frac{R_{2}^{i}}{R_{1}^{i}}+\left(n_{a}^{i} m_{a^{\prime}}^{i}+n_{a^{\prime}}^{i} m_{a}^{i}\right) \cos \alpha^{i}\right] I_{a a^{\prime}}^{j} I_{a a^{\prime}}^{l} \\
& =-2 N_{a} \sum_{\substack{(i, j, l)=\\
(1,2,3),(2,1,3),(3,1,2)}} \frac{1}{U_{2}^{i}}\left[\left(n_{a}^{i}+m_{a}^{i} U^{i}\right)\left(n_{a}^{i}+m_{a}^{i} \bar{U}^{i}\right)+\left(n_{a^{\prime}}^{i}+m_{a^{\prime}}^{i} \bar{U}^{i}\right)\left(n_{a^{\prime}}^{i}+m_{a^{\prime}}^{i} U^{i}\right)\right] I_{a a^{\prime}}^{j} I_{a a^{\prime}}^{l}
\end{aligned}
$$

Finally the relevant coefficient of (3.19) may be rewritten:

$$
\begin{aligned}
& \kappa_{a, \Omega \mathcal{R} \theta^{k} a}^{k}=-8 i N_{a} \rho_{\Omega \mathcal{R} \theta^{k}} I_{a ; O 6_{k}}\left[\operatorname{coth}\left(\pi v_{a}^{1 ; k}\right)+\operatorname{coth}\left(\pi v_{a}^{2 ; k}\right)+\operatorname{coth}\left(\pi v_{a}^{3 ; k}\right)\right] \\
& =8 N_{a} \rho_{\Omega \mathcal{R} \theta^{k}} \quad \sum_{(i, j, l)=} \frac{1}{\sin \alpha^{i}}\left[n_{O 6_{k}}^{i} n_{a}^{i} \frac{R_{1}^{i}}{R_{2}^{i}}+m_{O 6_{k}}^{i} m_{a}^{i} \frac{R_{2}^{i}}{R_{1}^{i}}\right. \\
& (1,2,3),(2,1,3),(3,1,2) \\
& \left.+\left(n_{a}^{i} m_{O 6_{k}}^{i}+n_{O 6_{k}}^{i} m_{a}^{i}\right) \cos \alpha^{i}\right] I_{a ; O 6_{k}}^{j} I_{a ; O 6_{k}}^{l} \\
& =4 N_{a} \rho_{\Omega \mathcal{R} \theta^{k}} \sum_{\substack{(i, j, l)=\\
(1,2,3),(2,1,3),(3,1,2)}} \frac{1}{U_{2}^{i}}\left[\left(n_{a}^{i}+m_{a}^{i} U^{i}\right)\left(n_{O 6_{k}}^{i}+m_{O 6_{k}}^{i} \bar{U}^{i}\right)\right. \\
& \left.+\left(n_{a}^{i}+m_{a}^{i} \bar{U}^{i}\right)\left(n_{O 6_{k}}^{i}+m_{O 6_{k}}^{i} U^{i}\right)\right] I_{a ; O 6_{k}}^{j} I_{a ; O 6_{k}}^{l} .
\end{aligned}
$$

The first observation we should make here is, that the tadpole contributions (3.40), (3.41), and (3.42) boil down to the $\mathrm{N}=2$ expressions (3.32), (3.36) and (3.39) after respecting the intersection properties in that case. That is why we have dropped the subscripts $\mathrm{N}=1$ at the $\delta$ 's in the above formulae. We shall use the latter in the following both for $\mathrm{N}=1$ and $\mathrm{N}=2$ sectors. Moreover, from the expression in appendix $\mathrm{C}$ we realize, that those parts of the Möbius $M_{a a^{\prime}}$ and the annulus diagram $A_{a b}$, which give rise to one-loop gauge corrections, may have only $N S$-tadpoles, but no $R$-tadpoles. This statement takes over to the annulus $A_{a a^{\prime}}$ diagram if only charges from one open string contribute (planar annulus), what precisley is the case in the other two diagrams mentioned before. We have seen from (3.23), that only charges from one string end contribute in the case of anomlay free gauge groups, i.e. in the case $E q$. (3.24) holds. Hence, the absence of $R$-tadpoles in one-loop gauge corrections is directly related to the absence of gauge anomalies. On the other hand, in the case of anomalous gauge groups in the non-planar annulus contribution of $\Delta_{a a^{\prime}}$ an $U V$ divergence, proportional to $\operatorname{Tr}(Q)$, appears from tadpoles of an anti-symmetric tensor field. This field couples to the background gauge field through a Green-Schwarz interaction. The situation is similar to what happens in the one-loop $F^{6}$ couplings of $S O(32)$ type $I$ string in $D=10$ : There the $U V$ divergence of a planar annulus diagram is cancelled against the divergence from the Möbius diagram. No potential gauge anomaly 
arises from these diagrams. On the other hand, the anomaly of the non-planar annulus diagram is cancelled through an exchange of an anti-symmetric tensor field in the closed string channel. This effect is seen as massles closed string pole in the non-planar annulus calculation.

All the coefficients of potential $N S$-contributions (3.40), (3.41) and (3.42) have to be summed up according to (3.1) and give a zero result:

$$
\sum_{\substack{b=1 \\ b \neq a}}^{K} \sum_{\substack{a \in[a] \\ b \in[b]}} \kappa_{a b}+\sum_{\substack{a, a^{\prime} \in[a] \\ a \neq a^{\prime}}} \kappa_{a a^{\prime}}+\sum_{\substack{a, a^{\prime} \in[a] \\ a \neq a^{\prime}}} \sum_{k=0}^{N-1} \kappa_{a, \Omega \mathcal{R} \theta^{k} a}^{k}=0
$$

This expression has to vanish in order that no $N S$-tadpoles appear in our one-loop gauge threshold calulation. In the following let us prove the validity of this equation for the $\mathbf{Z}_{2} \times \mathbf{Z}_{2}$ orientifold.

$\mathbf{Z}_{2} \times \mathbf{Z}_{2}$ orientifold:

As a concrete example, let us check the validity of (3.43) for the $\mathbf{Z}_{2} \times \mathbf{Z}_{2}$ orientifold. Type II $A$ compactified on a $\mathbf{Z}_{2} \times \mathbf{Z}_{2}$ orbifold, supplemented with the $\Omega \mathcal{R}$ orientifold projection (2.6), represents a rather simple background for intersecting branes to fulfill the requirements for vacuum tadpole cancellation and $\mathrm{N}=1$ chiral supersymmetry in $D=4$. These conditions are given by Eqs. (2.7) and (3.49). For details see Ref. [16,25]. The orbifold group is represented by the twists $\theta=\frac{1}{2}(1,-1,0)$ and $\omega=\frac{1}{2}(0,1,-1)$. We have the four $O 6$-planes $\Omega \mathcal{R}, \Omega \mathcal{R} \theta, \Omega \mathcal{R} \omega$, and $\Omega \mathcal{R} \theta \omega$. Their $R R$-charges are cancelled by introducing stacks $a$ of $D 6$ branes with wrapping numbers $\left(n_{a}^{j}, m_{a}^{j}\right)$ w.r.t. to the three internal tori $T_{2}^{j}$. The orbifold group generators $\theta$ and $\omega$ map each brane onto itself. In that case to each stack $a$ only one mirror $a^{\prime}=\mathcal{R} a$ is required. This means, that the conjugacy class of $a$ consists of only two elements, namely: $[a]=\{a, \mathcal{R} a\}$. Due to the simple structure of conjugacy classes the general formula (3.1) for the one-loop gauge corrections to the gauge group $G_{a}$ boils down to:

$$
\Delta_{G_{a}}=\sum_{\substack{b=1 \\ b \neq a}}^{K}\left(\Delta_{a b}+\Delta_{a b^{\prime}}+\Delta_{a^{\prime} b}+\Delta_{a^{\prime} b^{\prime}}\right)+\Delta_{a a^{\prime}}+2\left(\Delta_{a a^{\prime}}^{1}+\Delta_{a a^{\prime}}^{\theta}+\Delta_{a a^{\prime}}^{\omega}+\Delta_{a a^{\prime}}^{\theta \omega}\right)
$$

Note, that there a four possible twist insertions $1, \theta, \omega, \theta \omega$ into the Möbius diagram describing a string starting on a brane from stack $a$ and ending on its orientifold mirror $a^{\prime}$.

We shall investigate the cancellation of tadpoles in the gauge threshold result (3.44) for any intersecting brane setup which fulfills the requirements of vacuum tadpole cancellation. We first specialize to untilted tori $T_{2}^{j}$, i.e. the latter are chosen to be rectangular $\left(\alpha^{j}=\frac{\pi}{2}\right)$, 
though the generalization to tilted two-tori is straightforward and will be discussed at the end of this subsection. In that case, the orientifold mirror $a^{\prime}$ of a brane $a$ with wrapping numbers $\left(n_{a}^{j}, m_{a}^{j}\right)$ takes the wrapping numbers $\left(n_{a}^{j},-m_{a}^{j}\right)$. According to (3.40) an $\mathrm{N}=1$ annulus diagram, which describes a string starting on stack $a$, referring to the gauge group $G_{a}$ under consideration and which ends on stack $b$ from a different gauge group, contributes the coefficient

$$
\begin{aligned}
\sum_{\substack{b=1 \\
b \neq a}}^{K} \sum_{\substack{a \in\{a, \Omega \mathcal{R} a\} \\
b \in\{b, \Omega \mathcal{R} b\}}} \kappa_{a b} & =\sum_{\substack{b=1 \\
b \neq a}}^{K} \kappa_{a b}+\kappa_{a b^{\prime}}+\kappa_{a^{\prime} b}+\kappa_{a^{\prime} b^{\prime}} \\
& =-2 \sum_{\substack{b=1 \\
b \neq a}}^{K} \sum_{\substack{b \in\{b, \Omega \mathcal{R} b\} \\
a \in\{a, \Omega \mathcal{R} a\}}} N_{b} \sum_{\substack{(i, j, k)=\\
(1,2,3),(2,1,3),(3,1,2)}}\left(n_{a}^{i} n_{b}^{i} \frac{R_{1}^{i}}{R_{2}^{i}}+m_{a}^{i} m_{b}^{i} \frac{R_{2}^{i}}{R_{1}^{i}}\right) I_{a b}^{j} I_{a b}^{k} \\
& =8\left[\left(-n_{a}^{1} n_{a}^{2} n_{a}^{3} \frac{R_{1}^{1}}{R_{2}^{1}}+m_{a}^{1} m_{a}^{2} n_{a}^{3} \frac{R_{2}^{2}}{R_{1}^{2}}+m_{a}^{1} n_{a}^{2} m_{a}^{3} \frac{R_{2}^{3}}{R_{1}^{3}}\right) \sum_{\substack{b=1 \\
b \neq a}}^{K} N_{b} n_{b}^{1} m_{b}^{2} m_{b}^{3}\right. \\
& +\left(m_{a}^{1} m_{a}^{2} n_{a}^{3} \frac{R_{2}^{1}}{R_{1}^{1}}-n_{a}^{1} n_{a}^{2} n_{a}^{3} \frac{R_{1}^{2}}{R_{2}^{2}}+n_{a}^{1} m_{a}^{2} m_{a}^{3} \frac{R_{2}^{3}}{R_{1}^{3}}\right) \sum_{\substack{b=1 \\
b \neq a}}^{K} N_{b} m_{b}^{1} n_{b}^{2} m_{b}^{3} \\
& +\left(m_{a}^{1} n_{a}^{2} m_{a}^{3} \frac{R_{2}^{1}}{R_{1}^{1}}+n_{a}^{1} m_{a}^{2} m_{a}^{3} \frac{R_{2}^{2}}{R_{1}^{2}}-n_{a}^{1} n_{a}^{2} n_{a}^{3} \frac{R_{1}^{3}}{R_{2}^{3}}\right) \sum_{\substack{b=1 \\
b \neq a}}^{K} N_{b} m_{b}^{1} m_{b}^{2} n_{b}^{3} \\
& \left.-\left(n_{a}^{1} m_{a}^{2} m_{a}^{3} \frac{R_{1}^{1}}{R_{2}^{1}}+m_{a}^{1} n_{a}^{2} m_{a}^{3} \frac{R_{1}^{2}}{R_{2}^{2}}+m_{a}^{1} m_{a}^{2} n_{a}^{3} \frac{R_{1}^{3}}{R_{2}^{3}}\right) \sum_{\substack{b=1 \\
b \neq a}}^{K} N_{b} n_{b}^{1} n_{b}^{2} n_{b}^{3}\right]
\end{aligned}
$$

in front of the potential $U V$ divergence. Furthermore, an annulus diagram within an conjugacy class describes an open string exchange between stack $a$ and its mirror $a^{\prime}$ and leads to tadpole coefficient:

$$
\begin{aligned}
\kappa_{a a^{\prime}} & =-4 N_{a} \sum_{\substack{(i, j, k)=\\
(1,2,3),(2,1,3),(3,1,2)}}\left(n_{a}^{i} n_{a^{\prime}}^{i} \frac{R_{1}^{i}}{R_{2}^{i}}+m_{a}^{i} m_{a^{\prime}}^{i} \frac{R_{2}^{i}}{R_{1}^{i}}\right) I_{a a^{\prime}}^{j} I_{a a^{\prime}}^{k} \\
& =-16 N_{a}\left[m_{a}^{2} m_{a}^{3}\left(n_{a}^{1}\right)^{2} n_{a}^{2} n_{a}^{3} \frac{R_{1}^{1}}{R_{2}^{1}}+m_{a}^{1} m_{a}^{3} n_{a}^{1}\left(n_{a}^{2}\right)^{2} n_{a}^{3} \frac{R_{1}^{2}}{R_{2}^{2}}+m_{a}^{1} m_{a}^{2} n_{a}^{1} n_{a}^{2}\left(n_{a}^{3}\right)^{2} \frac{R_{1}^{3}}{R_{2}^{3}}\right. \\
& \left.-\left(m_{a}^{1}\right)^{2} m_{a}^{2} m_{a}^{3} n_{a}^{2} n_{a}^{3} \frac{R_{2}^{1}}{R_{1}^{1}}-m_{a}^{1}\left(m_{a}^{2}\right)^{2} m_{a}^{3} n_{a}^{1} n_{a}^{3} \frac{R_{2}^{2}}{R_{1}^{2}}-m_{a}^{1} m_{a}^{2}\left(m_{a}^{3}\right)^{2} n_{a}^{1} n_{a}^{2} \frac{R_{2}^{3}}{R_{1}^{3}}\right] .
\end{aligned}
$$


Finally, with the intersection numbers (2.26) and the general expression (3.42) for the Möbius divergence we obtain:

$$
\begin{aligned}
\kappa_{a a^{\prime}}^{1} & =-2^{6} i \rho_{\Omega \mathcal{R}} m_{a}^{1} m_{a}^{2} m_{a}^{3}\left[\operatorname{coth}\left(\pi v_{a}^{1}\right)+\operatorname{coth}\left(\pi v_{a}^{2}\right)+\operatorname{coth}\left(\pi v_{a}^{3}\right)\right] \\
& =2^{6} \rho_{\Omega \mathcal{R}}\left(n_{a}^{1} m_{a}^{2} m_{a}^{3} \frac{R_{1}^{1}}{R_{2}^{1}}+m_{a}^{1} n_{a}^{2} m_{a}^{3} \frac{R_{1}^{2}}{R_{2}^{2}}+m_{a}^{1} m_{a}^{2} n_{a}^{3} \frac{R_{1}^{3}}{R_{2}^{3}}\right), \\
\kappa_{a a^{\prime}}^{\theta} & =-2^{6} i \rho_{\Omega \mathcal{R} \theta} n_{a}^{1} n_{a}^{2} m_{a}^{3}\left[\tanh \left(\pi v_{a}^{1}\right)+\tanh \left(\pi v_{a}^{2}\right)+\operatorname{coth}\left(\pi v_{a}^{3}\right)\right] \\
& =2^{6} \rho_{\Omega \mathcal{R} \theta}\left(-m_{a}^{1} n_{a}^{2} m_{a}^{3} \frac{R_{2}^{1}}{R_{1}^{1}}-n_{a}^{1} m_{a}^{2} m_{a}^{3} \frac{R_{2}^{2}}{R_{1}^{2}}+n_{a}^{1} n_{a}^{2} n_{a}^{3} \frac{R_{1}^{3}}{R_{2}^{3}}\right), \\
\kappa_{a a^{\prime}}^{\omega} & =-2^{6} i \rho_{\Omega \mathcal{R} \omega} m_{a}^{1} n_{a}^{2} n_{a}^{3}\left[+\operatorname{coth}\left(\pi v_{a}^{1}\right)+\tanh \left(\pi v_{a}^{2}\right)+\tanh \left(\pi v_{a}^{3}\right)\right] \\
& =2^{6} \rho_{\Omega \mathcal{R} \omega}\left(n_{a}^{1} n_{a}^{2} n_{a}^{3} \frac{R_{1}^{1}}{R_{2}^{1}}-m_{a}^{1} m_{a}^{2} n_{a}^{3} \frac{R_{2}^{2}}{R_{1}^{2}}-m_{a}^{1} n_{a}^{2} m_{a}^{3} \frac{R_{2}^{3}}{R_{1}^{3}}\right), \\
\kappa_{a a^{\prime}}^{\theta \omega} & =-2^{6} i \rho_{\Omega \mathcal{R} \theta \omega} n_{a}^{1} m_{a}^{2} n_{a}^{3}\left[\tanh \left(\pi v_{a}^{1}\right)+\operatorname{coth}\left(\pi v_{a}^{2}\right)+\tanh \left(\pi v_{a}^{3}\right)\right] \\
& =2^{6} \rho_{\Omega \mathcal{R} \theta \omega}\left(-m_{a}^{1} m_{a}^{2} n_{a}^{3} \frac{R_{2}^{1}}{R_{1}^{1}}+n_{a}^{1} n_{a}^{2} n_{a}^{3} \frac{R_{1}^{2}}{R_{2}^{2}}-n_{a}^{1} m_{a}^{2} m_{a}^{3} \frac{R_{2}^{3}}{R_{1}^{3}}\right) .
\end{aligned}
$$

The phases resulting from the traces (2.11) may be taken from [13]: $\rho_{\Omega \mathcal{R} \theta}=\rho_{\Omega \mathcal{R} \omega}=$ $\rho_{\Omega \mathcal{R} \theta \omega}=-1$, and $\rho_{\Omega \mathcal{R}}=1$. With this information we add these tadpole contributions according to (3.44) and find a vanishing result:

$$
\delta_{G_{a}}=\kappa_{a b}+\kappa_{a b^{\prime}}+\kappa_{a^{\prime} b}+\kappa_{a^{\prime} b^{\prime}}+\kappa_{a a^{\prime}}+2\left(\kappa_{a a^{\prime}}^{1}+\kappa_{a a^{\prime}}^{\theta}+\kappa_{a a^{\prime}}^{\omega}+\kappa_{a a^{\prime}}^{\theta \omega}\right)=0 .
$$

To prove this identity, one only needs to apply the vacuum RR tadpole constraints [16]:

$$
\begin{array}{cc}
\sum_{a=1}^{K} N_{a} n_{a}^{1} n_{a}^{2} n_{a}^{3}=16 \quad & \sum_{a=1}^{K} N_{a} n_{a}^{1} m_{a}^{2} m_{a}^{3}=-16 \\
\sum_{a=1}^{K} N_{a} m_{a}^{1} n_{a}^{2} m_{a}^{3}=-16 & , \quad \sum_{a=1}^{K} N_{a} m_{a}^{1} m_{a}^{2} n_{a}^{3}=-16 .
\end{array}
$$

In addition the twisted tadpole conditions (2.7) have to hold, which enabled us to disregard $\theta, \omega, \theta \omega$-insertion in the annulus diagrams. The generalization to tilted tori is straightforward. Only the following modifications have to be performed in the Eqs. (3.45)-(3.47): changing $m_{a}^{j} \rightarrow \widetilde{m}_{a}^{j}=m_{a}^{j}+\frac{1}{2} n_{a}^{j}$ in the case of a tilted torus $T_{2}^{j}$, and replacing in $E q$. (3.47) the intersecting numbers for the untilted case with the relevant numbers (2.26) for the tilted case. Finally, to proof (3.48) for the tilted case, the vacuum tadpole condition for that case 25] has to be borrowed. In fact, we went through all these steps to prove (3.48) also for the tilted case. Hence in the $\mathbf{Z}_{2} \times \mathbf{Z}_{2}$ orbifold/orientifold with intersecting branes, the cancellation of vacuum tadpole contributions (3.49) implies the cancellation of tadpoles arising in an one-loop gauge threshold calculation. 


\subsection{General structure of one-loop gauge threshold corrections}

We have already explained in subsection 3.1, that due to the orbifold/orientifold action a stack $a$ of $N_{a}$ branes has several mirrors, all together summarized in the conjugacy class $[a]$. In orbifold/orientifold backgrounds with intersecting branes the spectrum and group representations of the open strings is organized according to which branes the open string ends couple.

From this analysis three different $\mathrm{N}=1$ open string sectors are possible. The open string sectors $a a, a^{\prime} a^{\prime}, \ldots$, with $a, a^{\prime}, \ldots \in[a]$, which describe open strings stretched between branes from stack $a, a^{\prime}, \ldots$ respectively, represent the vector multiplets describing the gauge group $G_{a}$. The latter is subject to the orbifold and orientifold action. In addition there are adjoint chiral matter fields coming from this sector. Since these strings are always stretched between two parallel branes, i.e. preserve $\mathrm{N}=4$ space-time supersymmetry, they are not relevant for gauge coupling renormalization. Open strings stretched between one brane $a$ of the gauge group $G_{a}$ and an other brane $b$ of a different gauge group $G_{b}$ give rise to $I_{a b}$ chiral fermions in the bifundamental representation of the gauge groups $G_{a}$ and $G_{b}$. Here $I_{a b}$ is the intersection number (2.3) of brane $a$ and $b$. More generally, after taking into account the various images contained in $[a]$ and $[b]$ we obtain from the open string sectors $a b+b a, a b^{\prime}+b^{\prime} a, a^{\prime} b+b a^{\prime}, \ldots$ chiral fermions in the bifundamental of the groups $G_{a}$ and $G_{b}$ with multiplicities $I_{a b}, I_{a b^{\prime}}, I_{a^{\prime} b}, \ldots$, respectively. We have seen, that those open string sectors contribute 3 to the one-loop gauge correction $\Delta_{G_{a}}$ the amount (cf. (3.16))

$$
\Delta_{a b}^{\mathrm{N}=1}=-b_{a b}^{N=1} \ln \frac{\Gamma\left(1-\frac{1}{\pi} \phi_{b a}^{1}\right) \Gamma\left(1-\frac{1}{\pi} \phi_{b a}^{2}\right) \Gamma\left(1-\frac{1}{\pi} \phi_{b a}^{3}\right)}{\Gamma\left(1+\frac{1}{\pi} \phi_{b a}^{1}\right) \Gamma\left(1+\frac{1}{\pi} \phi_{b a}^{2}\right) \Gamma\left(1+\frac{1}{\pi} \phi_{b a}^{3}\right)}
$$

with $\phi_{b a}^{j}$ being the angles between the branes $a$ and $b$. The latter respect 14 the $\mathrm{N}=1$ constraint $\phi_{b a}^{1}+\phi_{b a}^{2}+\phi_{b a}^{3}=0$. The coefficient (cf. (3.6))

$$
b_{a b}^{N=1}=N_{b} I_{a b} \operatorname{Tr}\left(Q_{a}^{2}\right)
$$

13 We do not display the potential divergences $\delta$ encountered in the previous subsections, as we have shown in subsection 3.4, that they are cancelled anyway after adding up all those potential tadpole contributions ( $c f . E q .(3.43)$ ).

14 Note, that according to the comments made before $E q$. 3.7 the angles $\phi_{b a}^{j}$ are assumed to be shifted back into the range $0<\left|\phi_{b a}^{j}\right|<\pi$. 
represents up to a sign the one-loop $\beta$-function coefficient $15 \beta_{a b}^{N=1}$. It precisely accounts for $I_{a b}$ bifundamental representations $\left(N_{a}, \bar{N}_{b}\right),\left(N_{a}, N_{b}\right), \ldots$ of the gauge groups $U\left(N_{a}\right) \times U\left(N_{b}\right)$. The latter is the gauge group arising from the stacks $a$ and $b$ before the orbifold and orientifold twists ( $c f$. also footnote 4 for the case, if one stack is parallel to an orientifold plane). Note, that a negative intersection number $I_{a b}$ gives rise to $-I_{a b}$ fermions of opposite chirality. Finally, open strings starting and ending on two different branes from one conjugacy class $[a]$, give rise to the sectors $a a^{\prime}+a^{\prime} a$, with $a, a^{\prime} \in[a]$. We obtain $I_{a a^{\prime}}$ symmetric and antisymmetric representations of $G_{a}$ from an open string stretched between the branes $a$ and $a^{\prime}$. Additional symmetric and antisymmetric representations of $G_{a}$ with multiplicity $I_{O 6_{k} ; a}$ arise, if $a^{\prime}=\Omega \mathcal{R} \theta^{k} a$. These sectors have annulus and Möbius diagrams contributing to the one-loop gauge correction $\Delta_{G_{a}}$, given by (3.28) and (3.21), respectively. With the relevant choice of angles $\phi_{a^{\prime} a}^{j}$ and $\phi_{a}^{j ; k}$ these corrections take the same form as (3.50) up to a modification of their coefficients 16 ( $c f$. Eqs. (3.26)

15 The field-theoretical one-loop $\beta$-function coefficient is obtained from the $I R$-limit $t \rightarrow \infty$ of the integrands (3.2) after converting them into the open string channel. For $\Delta_{a b}^{\mathrm{N}=1}$ this leads to:

$$
\Delta_{a b}^{\mathrm{N}=1}=\pi^{-1} i b_{a b}^{N=1} \int_{0}^{\infty} \frac{d t}{t}\left[\frac{\theta_{1}^{\prime}\left(\frac{v_{a b}^{1} t}{2}, \frac{i t}{2}\right)}{\theta_{1}\left(\frac{v_{a b}^{1} t}{2}, \frac{i t}{2}\right)}+\frac{\theta_{1}^{\prime}\left(\frac{v_{a b}^{2} t}{2}, \frac{i t}{2}\right)}{\theta_{1}\left(\frac{v_{a b}^{2} t}{2}, \frac{i t}{2}\right)}+\frac{\theta_{1}^{\prime}\left(\frac{v_{a b}^{3} t}{2}, \frac{i t}{2}\right)}{\theta_{1}\left(\frac{v_{a b}^{3} t}{2}, \frac{i t}{2}\right)}\right] .
$$

In the limit $t \rightarrow \infty$ the latter expression reproduces the one-loop running of the effective field theory $\left(c f\right.$. (1.2)). With $\lim _{t \rightarrow \infty} \frac{\theta_{1}^{\prime}\left(\frac{v t}{2}, \frac{i t}{2}\right)}{\theta_{1}\left(\frac{v t}{2}, \frac{v t}{2}\right)}=-(2 k-1) \pi i,(k-1)<-i v<k$, we verify $b_{a b}^{N=1}$ as being related to the correct $\beta$-function coefficient $\beta_{a b}^{N=1}$ through:

$$
\beta_{a b}^{N=1}= \begin{cases}-b_{a b}^{N=1}, & 0<\phi_{b a}^{1}+\phi_{b a}^{2}<\pi \\ b_{a b}^{N=1}, & \pi<\phi_{b a}^{1}+\phi_{b a}^{2}<2 \pi\end{cases}
$$

for the choice $0<\phi_{b a}^{1}, \phi_{b a}^{2}<\pi$. Note, that the sign flip in (3.53) is manifest in the logarithmic expression of (3.50).

16 For the Möbius diagram the transformation of (3.17) back into the open string sector yields:

$$
\Delta_{a, \Omega \mathcal{R} \theta^{k} a}^{k ; \mathrm{N}=1}=\pi^{-1} i b_{a, \Omega \mathcal{R} \theta^{k} a}^{k ; N=1} \int_{0}^{\infty} \frac{d t}{t}\left[\frac{\theta_{1}^{\prime}\left(v_{a}^{k ; 1} t, \frac{i t}{2}+\frac{1}{2}\right)}{\theta_{1}\left(v_{a}^{k ; 1} t, \frac{i t}{2}+\frac{1}{2}\right)}+\frac{\theta_{1}^{\prime}\left(v_{a}^{k ; 2} t, \frac{i t}{2}+\frac{1}{2}\right)}{\theta_{1}\left(v_{a}^{k ; 2} t, \frac{i t}{2}+\frac{1}{2}\right)}+\frac{\theta_{1}^{\prime}\left(v_{a}^{k ; 3} t, \frac{i t}{2}+\frac{1}{2}\right)}{\theta_{1}\left(v_{a}^{k ; 3} t, \frac{i t}{2}+\frac{1}{2}\right)}\right] .
$$

Hence the field-theoretical $\beta$-function coefficient is equal to $b_{a ; \Omega \mathcal{R} \theta^{k} a}^{N=1}$ up to a sign

$$
\beta_{a ; \Omega \mathcal{R} \theta^{k} a}^{N=1}= \begin{cases}-b_{a ; \Omega \mathcal{R} \theta^{k} a}^{N=1}, & 0<\phi_{a}^{k ; 1}+\phi_{a}^{k ; 2}<\frac{\pi}{2} \\ b_{a ; \Omega \mathcal{R} \theta^{k} a}^{N=1}, & \frac{\pi}{2}<\phi_{a}^{k ; 1}+\phi_{a}^{k ; 2}<\pi\end{cases}
$$

for the choice $0<\phi_{a}^{k ; 1}, \phi_{a}^{k ; 2}<\pi$. 
and $(3.18))$ :

$$
\begin{aligned}
b_{a a^{\prime}}^{N=1} & =2 N_{a} I_{a a^{\prime}} \operatorname{Tr}\left(Q_{a}^{2}\right), \\
b_{a ; \Omega \mathcal{R} \theta^{k} a}^{k ; N=1} & =-2 N_{a} \rho_{\Omega \mathcal{R} \theta^{k}} I_{a ; O 6_{k}} \operatorname{Tr}\left(Q_{a}^{2}\right)
\end{aligned}
$$

accounting for the number of possible symmetric and antisymmetric representations of the respective sector.

Two branes, which are parallel w.r.t. to one torus $T_{2}^{i}$, but have non-trivial intersections w.r.t. to the remaining two tori $T_{2}^{j}, T_{2}^{l}$, preserve $\mathrm{N}=2$ supersymmetry. Hence open string sectors associated to such branes give rise to $\mathrm{N}=2$ vectormultiplets and hypermultiplets. From the sectors $a b+b a, a b^{\prime}+b^{\prime} a, a^{\prime} b+b a^{\prime}, \ldots$ we obtain respectively $I_{a b}^{j} I_{a b}^{l}, I_{a b^{\prime}}^{j} I_{a b^{\prime}}^{l}, I_{a^{\prime} b}^{j} I_{a^{\prime} b}^{l}, \ldots$ copies of hypermultiplets in the bifundamental of the groups $G_{a}$ and $G_{b}$. To the full correction $\Delta_{G_{a}}$ these sectors constitute the one-loop gauge correction:

$$
\Delta_{a b}^{\mathrm{N}=2}=-b_{a b}^{N=2} \quad\left[\ln T_{2}^{i} V_{a}^{i}\left|\eta\left(T^{i}\right)\right|^{4}-\kappa\right]
$$

Here, $V_{a}^{i}$ represents the wrapped brane volume 2.20$)$

$$
V_{a}^{i}=\frac{1}{U_{2}^{i}}\left|n_{a}^{i}+U^{i} m_{a}^{i}\right|^{2}
$$

w.r.t. to the torus $T_{2}^{i}$ and the Kähler modulus $T^{i}$ is defined in (2.1). The one-loop $\beta$-function coefficient is given by (cf. Eq. (3.31)):

$$
b_{a b}^{N=2}=-2 N_{b} I_{a b}^{j} I_{a b}^{l} \operatorname{Tr}\left(Q_{a}^{2}\right)
$$

accounting for $I_{a b}^{j} I_{a b}^{l}$ bifundamental representations $\left(N_{a}, \bar{N}_{b}\right)$ of the gauge group $U\left(N_{a}\right) \times$ $U\left(N_{b}\right)$. This coefficient agrees with the field-theoretical $\mathrm{N}=2$ one-loop beta-function coefficient. Furthermore, the one-loop corrections from the open string sector $a a^{\prime}+a^{\prime} a$ take a similar form, with the $\beta$-function coefficients:

$$
\begin{aligned}
b_{a a^{\prime}}^{N=2} & =-4 N_{a} I_{a a^{\prime}}^{j} I_{a a^{\prime}}^{l} \operatorname{Tr}\left(Q_{a}^{2}\right), \\
b_{a ; \Omega \mathcal{R} \theta^{k} a}^{k ; N=2} & =8 N_{a} \rho_{\Omega \mathcal{R} \theta^{k}} I_{a ; O 6_{k}}^{j} I_{a ; O 6_{k}}^{l} \operatorname{Tr}\left(Q_{a}^{2}\right) .
\end{aligned}
$$

According to (3.1) the one-loop gauge threshold correction $\Delta_{G_{a}}$ to the gauge group $G_{a}$ is organized as a sum over all possible individual gauge corrections $\Delta_{a b}, \Delta_{a a^{\prime}}$ and $\Delta_{a ; \Omega \mathcal{R} \theta^{k} a}^{k}$ originating from the various open string sectors encountered before. Apart from the topological numbers (3.51), (3.56), (3.59) and (3.60) these corrections take a rather universal form, given by $(3.50)$ and (3.57). Hence the latter represent the two basic building blocks for the one-loop gauge correction $\Delta_{G_{a}}$, valid for any orbifold/orientifold backgrounds. It is quite reassuring, that the numbers (3.6), (3.18) and (3.26) arising from the string calculation precisely match (up to a sign) the field-theoretical expressions. The same applies 
for the $\mathrm{N}=2$ (string-theoretical) $\beta$-function coefficients (3.31), (3.35) and (3.38). To conclude, the gauge threshold correction $\Delta_{G_{a}}$ is fully determined by the two basic functions (3.50) and (3.57), the model dependent angles and moduli entering those functions and the field-theoretical $\beta$-function coefficients.

This is in close analogy to the results on the heterotic side [5] or for type $I$ orientifolds with non-intersecting $D 9$ and $D 5$ branes [18,19]. On the other hand, from the close analogy between one-loop gauge threshold corrections from the $\mathrm{N}=2$ sectors of intersecting branes, given by (3.57), and one-loop gauge threshold corrections from the $\mathrm{N}=2$ sectors of type $I$ orientifold compactifications, one would have been tempted to speculate, that one-loop gauge corrections from $\mathrm{N}=1$ sectors are also moduli independent constants, just as they are in type $I$ orbifold/orientifold compactifications with parallel branes [18, 19]. However, through (2.2) (or more precisely (3.11)) the angles $\phi_{a b}^{j}$ imply a non-trivial radius

$R_{1}^{j}, R_{2}^{j}$ dependence of (3.50), which we shall uncover in the next subsection. This leads to interesting moduli dependence of one-loop gauge corrections for $\mathrm{N}=1$ supersymmetric brane world models.

\subsection{Moduli dependence of $N=1$ gauge threshold corrections}

In ordinary orientifold compactifications, where the angles $\phi^{j}=i \pi v^{j}$ take discrete values, the $\theta$-functions in (3.4) do not give rise to any moduli dependence, and the $\mathrm{N}=1$ threshold correction $\Delta_{a b}^{\mathrm{N}=1}$ is just a constant, in agreement with the results of [18, 19. This is obvious from our general result (3.16), which holds for arbitrary orientifold/orbifold backgrounds with intersecting branes. The same applies for (3.21) and (3.28). However, in the case of branes at angles $\phi^{j}$, the angles are given through the relation (2.2). Therefore, the latter implies a non-trivial dependence of $\Delta_{a b}^{\mathrm{N}=1}$ on the radii $R_{1}^{j}, R_{2}^{j}$ encoded in (3.11). We want to determine this dependence in this subsection.

Rather than starting with (3.7) as before, we shall start with the relation

$$
\frac{\theta_{1}^{\prime}(i v, \tau)}{\theta_{1}(i v, \tau)}=-i \frac{\partial}{\partial v} \ln \theta_{1}(i v, \tau)=-i \frac{\pi}{F}-2 \pi i\left(F-\frac{1}{F}\right) \sum_{k=1}^{\infty} F^{2 k} C_{2 k}(\tau)
$$

which can be derived from Eq. (3.7) and:

$$
\pi v=\operatorname{arctanh} F \quad, \quad \frac{d F}{d v}=\pi\left(1-F^{2}\right) .
$$

Here, the functions $C_{2 k}(\tau)$ are given by 32

$$
C_{2 k}(\tau)=\sum_{n=1}^{\infty}\left(\frac{1+q^{n}}{1-q^{n}}\right)^{2 k}=\sum_{n=1}^{k} c(k, n)\left(1-E_{2 n}\right)
$$


with the coefficients:

$$
\begin{aligned}
& c(1,1)=\frac{1}{6}, \\
& c(2,1)=\frac{2}{9}, \quad c(2,2)=-\frac{1}{90}, \\
& c(3,1)=\frac{23}{90}, \quad c(3,2)=-\frac{1}{45}, c(3,3)=\frac{1}{945}, \\
& c(4,1)=\frac{88}{315}, c(4,2)=-\frac{22}{675}, c(4,3)=\frac{8}{2835}, c(4,4)=-\frac{1}{9450}, \quad \ldots .
\end{aligned}
$$

Inserting (3.61) into (3.4) we obtain:

$$
\Delta_{a b}^{\mathrm{N}=1}=\delta_{a b}^{\mathrm{N}=1}+4 i b_{a b}^{N=1} \int_{0}^{\infty} d l \sum_{j=1}^{3}\left(F_{a b}^{j}-\frac{1}{F_{a b}^{j}}\right) \sum_{k=1}^{\infty}\left(F_{a b}^{j}\right)^{2 k} C_{2 k}(\tau),
$$

with the background gauge fields

$$
\begin{aligned}
F_{a b}^{j} & =\tanh \left(\pi v_{a b}^{j}\right)=-i \tan \left(\phi_{b}^{j}-\phi_{a}^{j}\right) \\
& =-I_{a b}^{j} \frac{U^{j}-\bar{U}^{j}}{\left(n_{a}^{j}+m_{a}^{j} U^{j}\right)\left(n_{b}^{j}+m_{b}^{j} \bar{U}^{j}\right)+\left(n_{a}^{j}+m_{a}^{j} \bar{U}^{j}\right)\left(n_{b}^{j}+m_{b}^{j} U^{j}\right)},
\end{aligned}
$$

and the $U V$-divergent part $\delta_{a b}^{\mathrm{N}=1}$, given in (3.10), and eventually cancelled in the whole result (3.1). Using the integrals (3.13) we obtain:

$$
\begin{aligned}
\Delta_{a b}^{\mathrm{N}=1} & =\delta_{a b}^{\mathrm{N}=1}-4 \pi i b_{a b}^{N=1} \\
& \times \sum_{j=1}^{3}\left(F_{a b}^{j}-\frac{1}{F_{a b}^{j}}\right) \sum_{k=2}^{\infty}\left(F_{a b}^{j}\right)^{2 k} \sum_{n=2}^{k} \frac{(2 \pi)^{-2 n}(2 n) !}{(1-2 n)\left|B_{2 n}\right|} c(k, n) \zeta(2 n-1) \\
& =\delta_{a b}^{\mathrm{N}=1}+\frac{2 i}{3 \pi^{3}} b_{a b}^{N=1} \zeta(3) \sum_{j=1}^{3}\left(F_{a b}^{j}\right)^{3} \\
& +4 \pi i b_{a b}^{N=1} \sum_{k=2}^{\infty} \sum_{j=1}^{3}\left(F_{a b}^{j}\right)^{2 k+1}\left\{\sum_{n=2}^{k+1} \frac{(2 \pi)^{-2 n}(2 n) !}{(1-2 n)\left|B_{2 n}\right|} \zeta(2 n-1)[c(k+1, n)-c(k, n)]\right\} .
\end{aligned}
$$

Similar expressions can be derived for the other two corrections $\Delta_{a a^{\prime}}^{\mathrm{N}=1}$ and $\Delta_{a, \Omega \mathcal{R} \theta^{k} a}^{k ; \mathrm{N}=1}$.

The series in the expression (3.67) shows some interesting form, which may resemble its origin from $D=10$ type IIA string theory. In fact, in the one-loop $\mathrm{N}=2$ prepotential in $d=4$ (describing $F^{2}$ corrections [33]) or the $\mathrm{N}=1$ prepotential (describing $F^{4}$ corrections [34]) in $d=8$, there occurs a $\zeta(3)$ and $\zeta(5)$, respectively as a result of dimensional reducing higher gravitational couplings from $d=10$. Thus it is tempting to believe, that the coefficients $\zeta(2 n-1)$ in the series (3.67) also originate from higher gravitational couplings in $d=10$. Furthermore, the latter prepotentials have a geometric meaning in the corresponding dual string theory, which should also be the case here ( $c f$. the remarks in the conclusion). 


\subsection{Explicit results for the $\mathbf{Z}_{2} \times \mathbf{Z}_{2}$ orientifold}

In subsection 3.4 we have verified the absence of tadpoles in the one-loop gauge corrections (3.1) for the $\mathbf{Z}_{2} \times \mathbf{Z}_{2}$ orientifold with intersecting branes. This proof assumed an arbitrary choice of intersecting brane environment with the only condition on the latter, that it had to fulfill the vacuum tadpole conditions (3.49). In this subsection we take for the $\mathbf{Z}_{2} \times \mathbf{Z}_{2}$ orientifold a particular choice of intersecting branes, which obeys (3.49). For this model we shall calculate the corrections (3.1), which boil down to (3.44) for the $\mathbf{Z}_{2} \times \mathbf{Z}_{2}$ orientifold. More concretely, we shall study one of the concrete models presented in [16]. This represents a special $\mathrm{N}=1$ supersymmetric solution of the vacuum tadpole equations (3.49) with six stacks of $D 6$ branes, whose wrapping numbers are displayed in the following table:

\begin{tabular}{|l|l|lll|l|l|}
\hline Stack & Gauge group & $\phi_{a}^{1}$ & $\phi_{a}^{2}$ & $\phi_{a}^{3}$ & $N_{a}$ & SUSY \\
\hline 1 & $U(3) \times U(1)$ & $\arctan (2 U)$ & $-\arctan (2 U)$ & 0 & $6+2$ & $N=2$ \\
2 & $U(1)$ & 0 & 0 & 0 & 2 & $N=4$ \\
3 & $U S p(4)$ & 0 & $\arctan (2 U)$ & $-\arctan (2 U)$ & 4 & $N=2$ \\
4 & $U S p(8)$ & 0 & $\frac{\pi}{2}$ & $-\frac{\pi}{2}$ & 8 & $N=2$ \\
5 & $U(1)$ & $\arctan (4 U)$ & 0 & $-\arctan (4 U)$ & 2 & $N=2$ \\
6 & $U S p(8)$ & $\frac{\pi}{2}$ & 0 & $-\frac{\pi}{2}$ & 8 & $N=2$ \\
\hline
\end{tabular}

Table 1: D6 brane configuration: angles and supersymmetry w.r.t. the orientifold plane $\Omega \mathcal{R}$.

The $D 6$-branes are assumed to pass through fix points. The requirement for $\mathrm{N}=1$ supersymmetry leads to the choice of complex structure moduli: $U:=U^{1}=2 U^{2}=U^{3}$ in the three internal tori $T_{2}^{i}$. Furthermore for an untilted torus $a$ we have $\mu=0$ in (2.27).

As a concrete example we focus on the gauge group $U S p(4)$ referring to the third stack, with $N_{a}=4$. From the Table 1 we instantly see, that branes from that stack preserve at least $\mathrm{N}=2$ supersymmetry with their orientifold mirrors $a^{\prime}=\Omega \mathcal{R} a$. Hence only the correction $\Delta_{a a^{\prime}}^{N=2}$ has to be discussed for open string (annulus) exchanges between branes within the conjugacy class $[a]=\{a, \Omega \mathcal{R} a\}$. Using (3.34), $I_{a a^{\prime}}^{j}=-2 m_{a}^{j} n_{a}^{j}$, i.e. $I_{a a^{\prime}}^{2}=-4$ and $I_{a a^{\prime}}^{3}=2$ we obtain:

$$
\Delta_{a a^{\prime}}^{\mathrm{N}=2}=-32 N_{a} \operatorname{Tr}\left(Q_{U S p(4)}^{2}\right)\left[\ln \left|\eta\left(i R_{1}^{1} R_{2}^{1}\right)\right|^{4}+2 \ln \left(R_{1}^{1}\right)-\kappa\right] .
$$

Let us now determine the contributions from the Möbius sector. Without twist insertion, all the diagrams $M_{a, \Omega \mathcal{R} a}^{0}$ describe $\mathrm{N}=2$ supersymmetric open string exchanges from brane $a$ to its orientifold mirror $a^{\prime}=\Omega \mathcal{R} a$ and we directly get from (3.37)

$$
\begin{aligned}
\Delta_{a, \Omega \mathcal{R} a}^{0 ; \mathrm{N}=2} & =16 N_{a} n_{a}^{1} m_{a}^{2} m_{a}^{3} \operatorname{Tr}\left(Q_{U S p(4)}^{2}\right)\left[2 \ln R_{1}^{1}+\ln \left|\eta\left(i R_{1}^{1} R_{2}^{1}\right)\right|^{4}-\ln 2-\kappa\right] \\
& =-32 N_{a} \operatorname{Tr}\left(Q_{U S p(4)}^{2}\right)\left[2 \ln R_{1}^{1}+\ln \left|\eta\left(i R_{1}^{1} R_{2}^{1}\right)\right|^{4}-\ln 2-\kappa\right] .
\end{aligned}
$$


Furthermore, a twist insertions $\omega$ does not act in the first torus, which is responsible for the zero mode contributions of (3.69). Hence, in (3.69) we only have to change the intersection numbers referring to the second and third plane from $I_{a a^{\prime}}^{0 ; j}=2 m_{a}^{j}$ to $I_{a a^{\prime}}^{\omega ; 2}=2 n_{a}^{2}$ and $I_{a a^{\prime}}^{\omega ; 3}=-2 n_{a}^{3}$ to obtain:

$$
\begin{aligned}
\Delta_{a, \Omega \mathcal{R} a}^{\omega ; \mathrm{N}=2} & =-16 N_{a} n_{a}^{1} n_{a}^{2} n_{a}^{3} \operatorname{Tr}\left(Q_{U S p(4)}^{2}\right)\left[2 \ln R_{1}^{1}+\ln \left|\eta\left(i R_{1}^{1} R_{2}^{1}\right)\right|^{4}-\ln 2-\kappa\right] \\
& =-16 N_{a} \operatorname{Tr}\left(Q_{U S p(4)}^{2}\right)\left[2 \ln R_{1}^{1}+\ln \left|\eta\left(i R_{1}^{1} R_{2}^{1}\right)\right|^{4}-\ln 2-\kappa\right] .
\end{aligned}
$$

On the other hand, the twist insertions $\theta$ and $\theta \omega$ lead to $N=1$ Möbius diagrams, which after (3.21) assume the form:

$$
\begin{aligned}
\Delta_{a, \Omega \mathcal{R} a}^{\theta ; \mathrm{N}=1} & =-8 N_{a} n_{a}^{1} n_{a}^{2} m_{a}^{3} \operatorname{Tr}\left(Q_{U S p(4)}^{2}\right) \ln \frac{\Gamma\left(1-\frac{\phi_{a}^{1}}{\pi}+\frac{1}{2}\right) \Gamma\left(1-\frac{\phi_{a}^{2}}{\pi}-\frac{1}{2}\right) \Gamma\left(1-\frac{\phi_{a}^{3}}{\pi}\right)}{\Gamma\left(1+\frac{\phi_{a}^{1}}{\pi}-\frac{1}{2}\right) \Gamma\left(1+\frac{\phi_{a}^{2}}{\pi}+\frac{1}{2}\right) \Gamma\left(1+\frac{\phi_{a}^{3}}{\pi}\right)} \\
& =8 N_{a} \operatorname{Tr}\left(Q_{U S p(4)}^{2}\right) \ln \frac{1}{2} \frac{\Gamma\left[\frac{1}{2}-\frac{1}{\pi} \arctan (2 U)\right] \Gamma\left[1+\frac{1}{\pi} \arctan (2 U)\right]}{\Gamma\left[\frac{3}{2}+\frac{1}{\pi} \arctan (2 U)\right] \Gamma\left[1-\frac{1}{\pi} \arctan (2 U)\right]} \\
\Delta_{a, \Omega \mathcal{R} a}^{\theta \omega ; \mathrm{N}=1} & =-8 N_{a} n_{a}^{1} m_{a}^{2} n_{a}^{3} \operatorname{Tr}\left(Q_{U S p(4)}^{2}\right) \ln \frac{\Gamma\left(1-\frac{\phi_{a}^{1}}{\pi}+\frac{1}{2}\right) \Gamma\left(1-\frac{\phi_{a}^{2}}{\pi}\right) \Gamma\left(1-\frac{\phi_{a}^{3}}{\pi}-\frac{1}{2}\right)}{\Gamma\left(1+\frac{\phi_{a}^{1}}{\pi}-\frac{1}{2}\right) \Gamma\left(1+\frac{\phi_{a}^{2}}{\pi}\right) \Gamma\left(1+\frac{\phi_{a}^{3}}{\pi}+\frac{1}{2}\right)} \\
& =-16 N_{a} \operatorname{Tr}\left(Q_{U S p(4)}^{2}\right) \ln \frac{1}{2} \frac{\Gamma\left[1-\frac{1}{\pi} \arctan (2 U)\right] \Gamma\left[\frac{1}{2}+\frac{1}{\pi} \arctan (2 U)\right]}{\Gamma\left[1+\frac{1}{\pi} \arctan (2 U)\right] \Gamma\left[\frac{3}{2}-\frac{1}{\pi} \arctan (2 U)\right]} .
\end{aligned}
$$

Let us now come to the annulus contributions from open strings starting on branes $a$ or their mirrors from the stack constituing the gauge group $U S p(4)$ and ending on branes $b$ or their mirrors from different stacks. Summing up all $\mathrm{N}=2$ sectors gives rise to

$$
\begin{aligned}
\Delta_{a b}^{N=2} & =32 N_{a} \operatorname{Tr}\left(Q_{U S p(4)}^{2}\right)\left[-6 \ln R_{1}^{1}-2 \ln R_{1}^{2}-2 \ln R_{2}^{2}-\ln \left(\frac{R_{1}^{2}}{R_{2}^{2}}+4 \frac{R_{2}^{2}}{R_{1}^{2}}\right)\right. \\
& \left.-3 \ln \left|\eta\left(i R_{1}^{1} R_{2}^{1}\right)\right|^{4}-\ln \left|\eta\left(i R_{1}^{2} R_{2}^{2}\right)\right|^{4}+4 \kappa\right],
\end{aligned}
$$

whereas the $\mathrm{N}=1$ sectors sum up to:

$$
\begin{aligned}
\Delta_{a b}^{N=1} & =N_{a} \operatorname{Tr}\left(Q_{U S p(4)}^{2}\right)\left\{256 \ln \frac{\Gamma\left[1-\frac{1}{\pi} \arctan (2 U)\right]}{\Gamma\left[1+\frac{1}{\pi} \arctan (2 U)\right]}+32 \ln \frac{\Gamma\left[\frac{1}{2}+\frac{1}{\pi} \arctan (2 U)\right]}{\Gamma\left[\frac{1}{2}-\frac{1}{\pi} \arctan (2 U)\right]}\right. \\
& +32 \ln \frac{\Gamma\left[\frac{3}{2}+\frac{1}{\pi} \arctan (2 U)\right]}{\Gamma\left[\frac{3}{2}-\frac{1}{\pi} \arctan (2 U)\right]}+64 \ln \frac{\Gamma\left[1+2 \frac{1}{\pi} \arctan (2 U)\right]}{\Gamma\left[1-2 \frac{1}{\pi} \arctan (2 U)\right]} \\
& +32 \ln \frac{\Gamma\left[1-\frac{1}{\pi} \arctan (4 U)\right]}{\Gamma\left[1+\frac{1}{\pi} \arctan (4 U)\right]}+16 \ln \frac{\Gamma\left[1+\frac{1}{\pi} \arctan (2 U)-\frac{1}{\pi} \arctan (4 U)\right]}{\Gamma\left[1-\frac{1}{\pi} \arctan (2 U)+\frac{1}{\pi} \arctan (4 U)\right]} \\
& \left.+48 \ln \frac{\Gamma\left[1+\frac{1}{\pi} \arctan (2 U)+\frac{1}{\pi} \arctan (4 U)\right]}{\Gamma\left[1-\frac{1}{\pi} \arctan (2 U)-\frac{1}{\pi} \arctan (4 U)\right]}\right\} .
\end{aligned}
$$

Finally summing up all seven contributions (3.69)-(3.73) gives the one-loop gauge threshold correction $\Delta_{U S p(4)}$ to the gauge group $U S p(4)$ :

$$
\Delta_{U S p(4)}=2 \Delta_{a, \Omega \mathcal{R} a}^{\theta ; \mathrm{N}=1}+2 \Delta_{a, \Omega \mathcal{R} a}^{\theta \omega ; \mathrm{N}=1}+\Delta_{a b}^{N=1}+\Delta_{a a^{\prime}}^{\mathrm{N}=2}+2 \Delta_{a, \Omega \mathcal{R} a}^{0 ; \mathrm{N}=2}+2 \Delta_{a, \Omega \mathcal{R} a}^{\omega ; \mathrm{N}=2}+\Delta_{a b}^{N=2} .
$$




\section{Conclusions}

We have calculated the one-loop corrections to gauge couplings in $\mathrm{N}=1$ supersymmetric brane world models. These models are realized through stacks of $D 6$ branes, which are placed into an orbifold/orientifold background of type $I I A$ string theory and wrapped on 3-cycles with non-vanishing intersections. The one-loop gauge thresholds are organized as a sum (3.1) over the corrections coming from the individual open string sectors stretched between the various intersecting $D 6$-branes. The correction associated to one of this sector takes a universal form given by the one-loop $\beta$-function coefficient multiplied by one of the two basic functions (3.50) and (3.57). The moduli dependence of this sector enters these functions through the respective angles describing the open string sector (encoded in (3.11) ). Hence the complete correction $\Delta_{G_{a}}$ is fully determined by the two functions (3.50) and (3.57), the model dependent angles and moduli entering those functions and the field-theoretical $\beta$-function coefficients.

The supersymmetric orientifold models considered here map in the strong coupling limit to compactifications of $M$-theory on certain singular $G_{2}$ manifolds. Thus our $\mathrm{N}=1$ gauge threshold function (3.50) is possibly related to the recently calculated Ray-Singer torsion of singular $G_{2}$ manifolds [35]. Besides (3.50) may give a hint on the form of non-perturbative corrections to the gauge couplings on the heterotic side. In view of Refs. [36,37 the correction (3.50) represents an other class of function, which describes one-loop couplings with only $1 / 4$ BPS states contributing and which maps to non-perturbative corrections on the dual heterotic side. Hence the object entering the integrand (3.4) represents a (weighted) counting function of these states, which is mapped on the dual heterotic side to a possible topological quantity, associated to the singular $G_{2}$ compactification manifold.

Since the angles are related to the radii of the type $I I A$ compactification through (3.66) the angles imply a non-trivial radius dependence, shown in (3.67). This has the consequence that, in contrast to what is known from ordinary orbifold/orientifold theories, $\mathrm{N}=1$ subsectors do give rise to moduli-dependent one-loop corrections, which may become huge for certain regions in the moduli space. This fact has an important impact on the unification scale and other phenomenological properties in intersecting brane world models [8.

\section{Acknowledgments}

We wish to thank R. Blumenhagen, L. Görlich, G. Honecker, and T. Ott for numerous and valuable discussions. This work is supported in part by the Deutsche Forschungsgemeinschaft (DFG), and the German-Israeli Foundation (GIF). St.St. thanks the CERN Theory Division for hospitality during completion of this work. 


\section{Appendix A. Modular functions and Dirichlet series}

In this appendix we want to investigate the following type of integrals

$$
\int_{0}^{\infty} d y y^{s-1}\left[E_{2 k}(i y)-1\right]
$$

as they appear in the calculation of one-loop gauge threshold corrections for $\mathrm{N}=1$ sectors (cf. (3.65)). Essentially we shall prove Eq. (3.13). This leads us to the connection between a modular form with Fourier series

$$
f(\tau)=c(0)+\sum_{n=1}^{\infty} c(n) e^{2 \pi i n \tau}
$$

and the Dirichlet series

$$
D(s)=\sum_{n=1}^{\infty} \frac{c(n)}{n^{s}}
$$

which was established by Hecke, see e.g. [38]. Let us review the relevant steps. If $f$ is an element of the set of all entire modular forms of weight $2 k$, its Fourier coefficients fulfill $c(n) \sim \mathcal{O}\left(n^{2 k-1}\right)$. Hence the series (A.3) is absolutely convergent for $\operatorname{Re}(s)>2 k$. For $k>1$ the isomorphism between (A.2) and (A.3) is made transparent through the integral:

$$
(2 \pi n)^{-s} \Gamma(s)=\int_{0}^{\infty} y^{s-1} e^{-2 \pi n y} d y \quad, \quad \operatorname{Re}(s)>0 .
$$

With multiplying both sides by $c(n)$ and sum over $n$ we obtain:

$$
(2 \pi)^{-s} \Gamma(s) D(s)=\int_{0}^{\infty} y^{s-1}[f(i y)-c(0)] d y .
$$

In the above step we exchanged the order of summation and integration, which is only valid for $\operatorname{Re}(s)>2 k$. However, after explicitly evaluating 17 the integral on the r.h.s.

$$
(2 \pi)^{-s} \Gamma(s) D(s)=\int_{1}^{\infty}\left[y^{s}+(-1)^{k} y^{2 k-s}\right][f(i y)-c(0)] d y-c(0)\left(\frac{1}{s}+\frac{(-1)^{k}}{2 k-s}\right)
$$

17 This makes use of the modular behaviour of $f$, which is guaranteed for $k>1$, what is our assumption. Note, that there does not exist a modular function of weight 2 . 
one may obtain an analytic continuation to the region $\operatorname{Re}(s)<2 k$ with $D(s)$ fulfilling the following functional equation [38]:

$$
(2 \pi)^{-s} \Gamma(s) D(s)=(-1)^{k}(2 \pi)^{s-2 k} \Gamma(2 k-s) D(2 k-s) .
$$

At $s=2 k$ there is a pole with residue:

$$
\frac{(-1)^{k}(2 \pi)^{2 k} c(0)}{\Gamma(2 k)} .
$$

The procedure outlined above may be applied for the Eisenstein series

$$
E_{2 k}(\tau)=1+\frac{(2 \pi i)^{2 k}}{(2 k-1) ! \zeta(2 k)} \sum_{n=1}^{\infty} \sigma_{2 k-1}(n) e^{2 \pi i n \tau} \quad, \quad k \geq 1
$$

with:

$$
\sigma_{2 k-1}(n)=\sum_{\substack{d=1 \\ d \mid n}}^{\infty} d^{2 k-1}
$$

The Dirichlet series associated to the function $f(\tau)=E_{2 k}(\tau)$ may be obtained from (A.5) for $\operatorname{Re}(s)>2 k$. With using (A.4) we may evaluate the following integral for $s>0$

$$
\begin{aligned}
(2 \pi)^{-s} \Gamma(s) D(s) & =\int_{0}^{\infty} y^{s-1}\left[E_{2 k}(i y)-1\right] d y \\
& =\frac{(2 \pi i)^{2 k}}{(2 k-1) ! \zeta(2 k)} \Gamma(s) \sum_{n=1}^{\infty} \sigma_{2 k-1}(n)(2 \pi n)^{-s} \\
& =\frac{(2 \pi i)^{2 k}}{(2 k-1) ! \zeta(2 k)} \Gamma(s) \sum_{m=1}^{\infty} \sum_{d=1}^{\infty} d^{2 k-1}(2 \pi d m)^{-s} \\
& =\frac{(2 \pi i)^{2 k}}{(2 k-1) ! \zeta(2 k)}(2 \pi)^{-s} \Gamma(s) \zeta(1+s-2 k) \zeta(s) .
\end{aligned}
$$

Thus, for $\operatorname{Re}(s)>2 k$ we obtain the Dirichlet series

$$
D(s)=\frac{(2 \pi i)^{2 k}}{(2 k-1) ! \zeta(2 k)} \zeta(1+s-2 k) \zeta(s), \quad \operatorname{Re}(s)>2 k,
$$

associated to the modular function $E_{2 k}$. However, if in addition $k>1$, the relation (A.7) may be applied to give us the analytic continuation of (A.12) to the region $\operatorname{Re}(s)<2 k$ :

$$
\begin{aligned}
D(s) & =(-1)^{k}(2 \pi)^{2 s-2 k} \frac{\Gamma(2 k-s)}{\Gamma(s)} D(2 k-s) \\
& =\frac{(2 \pi i)^{2 k}}{(2 k-1) ! \zeta(2 k)}(-1)^{k}(2 \pi)^{2 s-2 k} \frac{\Gamma(2 k-s)}{\Gamma(s)} \zeta(1-s) \zeta(2 k-s) \quad, \quad \operatorname{Re}(s)<2 k .
\end{aligned}
$$


The previous equations allow us to extract an expression for the integral (A.1):

$$
\begin{aligned}
& \int_{0}^{\infty} d y y^{s-1}\left[E_{2 k}(i y)-1\right] \\
& \quad= \begin{cases}\frac{(2 \pi i)^{2 k}}{(2 k-1) ! \zeta(2 k)}(2 \pi)^{-s} \Gamma(s) \zeta(1+s-2 k) \zeta(s), & \operatorname{Re}(s)>2 k, \\
\frac{(2 \pi i)^{2 k}}{(2 k-1) ! \zeta(2 k)}(-1)^{k}(2 \pi)^{s-2 k} \Gamma(2 k-s) \zeta(1-s) \zeta(2 k-s), & \operatorname{Re}(s)<2 k .\end{cases}
\end{aligned}
$$

In particular, the case $s=1$ leads to $(k>1)$ :

$$
\begin{aligned}
\int_{0}^{\infty} d y\left[E_{2 k}(i y)-1\right] & =-\frac{1}{2} \frac{(2 \pi i)^{2 k}}{(2 k-1) ! \zeta(2 k)}(-1)^{k}(2 \pi)^{1-2 k} \Gamma(2 k-1) \zeta(2 k-1) \\
& =\frac{\pi}{(1-2 k) \zeta(2 k)} \zeta(2 k-1) .
\end{aligned}
$$

This result may be also directly anticipated from regulating the integral (A.11). Indeed replacing in (A.11) the integer $s$ by $1+\epsilon$, with $\epsilon>0$ playing the role of a regulator, we obtain:

$$
\begin{aligned}
& \int_{0}^{\infty} d y y^{\epsilon}\left[E_{2 k}(i y)-1\right]=\frac{(2 \pi i)^{2 k}}{(2 k-1) ! \zeta(2 k)}(2 \pi)^{-1-\epsilon} \Gamma(1+\epsilon) \zeta(2+\epsilon-2 k) \zeta(1+\epsilon) \\
& \quad= \begin{cases}-\frac{1}{2} \frac{(2 \pi i)^{2 k}}{(2 k-1) ! \zeta(2 k)}(-1)^{k}(2 \pi)^{1-2 k} \Gamma(2 k-1) \zeta(2 k-1)+\mathcal{O}(\epsilon), & k>1, \\
\frac{6}{\pi \epsilon}+\mathcal{O}(\epsilon), & k=1 .\end{cases}
\end{aligned}
$$

The case $k>1$, clearly agrees with (A.15) in the limit $\epsilon \rightarrow 0$. However, in addition (A.16) yields an (regularized) expression for the case $k=1$.

Similarly, for the Möbius diagram we need the integral:

$$
\begin{aligned}
\int_{0}^{\infty} d y y^{\epsilon} & {\left[E_{2 k}\left(i y-\frac{1}{2}\right)-1\right] } \\
= & \frac{(2 \pi i)^{2 k}}{(2 k-1) ! \zeta(2 k)}(2 \pi)^{-1-\epsilon} \Gamma(1+\epsilon) \zeta(2+\epsilon-2 k) \zeta(1+\epsilon) \Pi(\epsilon, k) \\
= & \begin{cases}-\frac{1}{2} 2^{2 k-4} \frac{(2 \pi i)^{2 k}}{(2 k-1) ! \zeta(2 k)}(-1)^{k}(2 \pi)^{1-2 k} \Gamma(2 k-1) \zeta(2 k-1)+\mathcal{O}(\epsilon), & k>1, \\
\frac{3}{2 \pi \epsilon}+\frac{3 \ln 2}{\pi}+\mathcal{O}(\epsilon), & k=1 .\end{cases}
\end{aligned}
$$

The projector 18

$$
\Pi(\epsilon, k)=2^{-1+2 k-\epsilon}+2^{-\epsilon}-7 \cdot 4^{-2+k-\epsilon}-1
$$

18 In Eq. (A.11) it manifests in the sum over $m$ and $d$ as $\frac{1}{2}\left[1+(-1)^{m}\right]\left[1+(-1)^{d}\right]-(-1)^{d+m}$. 
steams from the $\frac{1}{2}$ in the argument of the Eisenstein function. Thus the effect of the latter is the additional factor $2^{2 k-4}$ and a slight modification in the regularization of the case $k=1$ compared to (A.16).

\section{Appendix B. Spin-structure sums in the gauged open string partition function}

To perform the even spin-structure sum of (2.29), supplemented with the relevant piece at $\mathcal{O}\left(B^{2}\right)$ order $(3.3)$, the following identity, which follows from applying the Riemann identity (cf. e.g. [39]), is useful:

$$
\begin{aligned}
\frac{\partial^{2}}{\partial z^{2}} \sum_{\vec{\delta}} s_{\vec{\delta}} & \left.\theta\left[\begin{array}{l}
\delta_{1} \\
\delta_{2}
\end{array}\right](z, \tau) \theta\left[\begin{array}{l}
\delta_{1} \\
\delta_{2}
\end{array}\right]\left(v^{1}, \tau\right) \theta\left[\begin{array}{l}
\delta_{1} \\
\delta_{2}
\end{array}\right]\left(v^{2}, \tau\right) \theta\left[\begin{array}{l}
\delta_{1} \\
\delta_{2}
\end{array}\right]\left(v^{3}, \tau\right)\right|_{z=0} \\
& =\frac{1}{2} \theta_{1}^{\prime \prime}\left[\frac{v^{1}+v^{2}+v^{3}}{2}\right] \theta_{1}\left[\frac{v^{1}+v^{2}-v^{3}}{2}\right] \theta_{1}\left[\frac{v^{1}-v^{2}+v^{3}}{2}\right] \theta_{1}\left[\frac{v^{1}-v^{2}-v^{3}}{2}\right] \\
& +\frac{1}{2} \theta_{1}\left[\frac{v^{1}+v^{2}+v^{3}}{2}\right] \theta_{1}^{\prime \prime}\left[\frac{v^{1}+v^{2}-v^{3}}{2}\right] \theta_{1}\left[\frac{v^{1}-v^{2}+v^{3}}{2}\right] \theta_{1}\left[\frac{v^{1}-v^{2}-v^{3}}{2}\right] \\
& +\frac{1}{2} \theta_{1}\left[\frac{v^{1}+v^{2}+v^{3}}{2}\right] \theta_{1}\left[\frac{v^{1}+v^{2}-v^{3}}{2}\right] \theta_{1}^{\prime \prime}\left[\frac{v^{1}-v^{2}+v^{3}}{2}\right] \theta_{1}\left[\frac{v^{1}-v^{2}-v^{3}}{2}\right] \\
& -\frac{1}{2} \theta_{1}\left[\frac{v^{1}+v^{2}+v^{3}}{2}\right] \theta_{1}\left[\frac{v^{1}+v^{2}-v^{3}}{2}\right] \theta_{1}\left[\frac{v^{1}-v^{2}+v^{3}}{2}\right] \theta_{1}^{\prime \prime}\left[\frac{v^{1}-v^{2}-v^{3}}{2}\right] \\
& -\theta_{1}^{\prime}\left[\frac{v^{1}+v^{2}+v^{3}}{2}\right] \theta_{1}^{\prime}\left[\frac{v^{1}+v^{2}-v^{3}}{2}\right] \theta_{1}\left[\frac{v^{1}-v^{2}+v^{3}}{2}\right] \theta_{1}\left[\frac{v^{1}-v^{2}-v^{3}}{2}\right] \\
& +\theta_{1}^{\prime}\left[\frac{v^{1}+v^{2}+v^{3}}{2}\right] \theta_{1}\left[\frac{v^{1}+v^{2}-v^{3}}{2}\right] \theta_{1}^{\prime}\left[\frac{v^{1}-v^{2}+v^{3}}{2}\right] \theta_{1}\left[\frac{v^{1}-v^{2}-v^{3}}{2}\right] \\
& +\theta_{1}^{\prime}\left[\frac{v^{1}+v^{2}+v^{3}}{2}\right] \theta_{1}\left[\frac{v^{1}+v^{2}-v^{3}}{2}\right] \theta_{1}\left[\frac{v^{1}-v^{2}+v^{3}}{2}\right] \theta_{1}^{\prime}\left[\frac{v^{1}-v^{2}-v^{3}}{2}\right] \\
& +\theta_{1}\left[\frac{v^{1}+v^{2}+v^{3}}{2}\right] \theta_{1}^{\prime}\left[\frac{v^{1}+v^{2}-v^{3}}{2}\right] \theta_{1}^{\prime}\left[\frac{v^{1}-v^{2}+v^{3}}{2}\right] \theta_{1}\left[\frac{v^{1}-v^{2}-v^{3}}{2}\right] \\
& -\theta_{1}\left[\frac{v^{1}+v^{2}+v^{3}}{2}\right] \theta_{1}^{\prime}\left[\frac{v^{1}+v^{2}-v^{3}}{2}\right] \theta_{1}\left[\frac{v^{1}-v^{2}+v^{3}}{2}\right] \theta_{1}^{\prime}\left[\frac{v^{1}-v^{2}-v^{3}}{2}\right] \\
& -\theta_{1}\left[\frac{v^{1}+v^{2}+v^{3}}{2}\right] \theta_{1}\left[\frac{v^{1}+v^{2}-v^{3}}{2}\right]
\end{aligned}
$$

Let us notify the identity $\theta_{1}^{\prime}(0, \tau)=-2 \pi \eta(\tau)^{3}$, which will be used in the following.

\section{B.1. $N=1$ supersymmetric sector: $v^{j} \neq 0$ and $\pm v^{1} \pm v^{2} \pm v^{3}=0$}

In this case $\mathrm{N}=1$ supersymmetry is preserved and one-loop corrections to gauge couplings are generically non-vanishing. Using (3.3) and (B.1) the part of (2.29), which is 
relevant for the gauge threshold corrections becomes:

$$
\begin{aligned}
\left.\frac{\partial^{2}}{\partial B^{2}} \widetilde{A}_{a b}^{d=3}(B)\right|_{B=0} & =\frac{1}{2} q_{a}^{2} I_{a b} l \frac{1}{\eta(\tau)^{3}} \\
& \times \sum_{\vec{\delta}} s_{\vec{\delta}} \theta^{\prime \prime}\left[\begin{array}{l}
\delta_{1} \\
\delta_{2}
\end{array}\right](0, \tau) \frac{\theta\left[\begin{array}{l}
\delta_{1} \\
\delta_{2}
\end{array}\right]\left(i v^{1}, \tau\right)}{\theta\left[\begin{array}{l}
\frac{1}{2} \\
\frac{1}{2}
\end{array}\right]\left(i v^{1}, \tau\right)} \frac{\theta\left[\begin{array}{l}
\delta_{1} \\
\delta_{2}
\end{array}\right]\left(i v^{2}, \tau\right) \theta\left[\begin{array}{l}
\delta_{1} \\
\delta_{2}
\end{array}\right]\left(i v^{3}, \tau\right)}{\theta\left[\begin{array}{l}
\frac{1}{2} \\
\frac{1}{2}
\end{array}\right]\left(i v^{3}, \tau\right)} \frac{\theta\left[\begin{array}{l}
\frac{1}{2} \\
\frac{1}{2}
\end{array}\right]\left(i v^{3}, \tau\right)}{\theta_{1}^{\prime}\left(i v^{2}, \tau\right)} \\
& =-\pi q_{a}^{2} I_{a b} l(-1)^{\#}\left[\frac{\theta_{1}^{\prime}\left(i v^{3}, \tau\right)}{\theta_{1}\left(i v^{1}, \tau\right)} \pm .\right.
\end{aligned}
$$

with \# accounting for the number of minus signs in the combination $\pm v^{1} \pm v^{2} \pm v^{3}$ and the modular parameter $\tau=2 i l$ for the annulus in the closed string channel.

\section{B.2. $N=2$ supersymmetric sector: $v^{i}, v^{j} \neq 0, v^{k}=0$ and $\pm v^{i} \pm v^{j}=0$}

In this case $\mathrm{N}=2$ supersymmetry is preserved and again one-loop corrections to gauge couplings are generically non-vanishing. Using (3.3) and (B.1) the piece of the gauged open string partition function (2.29), needed for the gauge threshold corrections becomes

$$
\begin{aligned}
\left.\frac{\partial^{2}}{\partial B^{2}} \widetilde{A}_{a b}^{d=2}(B)\right|_{B=0} & =-\frac{1}{4} q_{a}^{2} V_{a}^{k} I_{a b}^{i} I_{a b}^{j} l \frac{1}{\eta(\tau)^{6}} \widetilde{Z}_{k}\left(l, T^{k}, V^{k}\right) \\
& \times \sum_{\vec{\delta}} s_{\vec{\delta}} \theta^{\prime \prime}\left[\begin{array}{l}
\delta_{1} \\
\delta_{2}
\end{array}\right](0, \tau) \theta\left[\begin{array}{l}
\delta_{1} \\
\delta_{2}
\end{array}\right](0, \tau) \frac{\theta\left[\begin{array}{l}
\delta_{1} \\
\delta_{2}
\end{array}\right]\left(i v^{i}, \tau\right) \theta\left[\begin{array}{l}
\delta_{1} \\
\delta_{2}
\end{array}\right]\left(i v^{j}, \tau\right)}{\theta\left[\begin{array}{l}
\frac{1}{2} \\
\frac{1}{2}
\end{array}\right]\left(i v^{i}, \tau\right)} \theta\left[\begin{array}{c}
\frac{1}{2} \\
\frac{1}{2}
\end{array}\right]\left(i v^{j}, \tau\right) \\
& =-\pi^{2} q_{a}^{2} V_{a}^{k} I_{a b}^{i} I_{a b}^{j} l \widetilde{Z}_{k}\left(l, T^{k}, V_{a}^{k}\right) .
\end{aligned}
$$

For the spin-structure sums in $\left.\frac{\partial^{2}}{\partial B^{2}} \widetilde{M}_{a}(B)\right|_{B=0}$ and $\left.\frac{\partial^{2}}{\partial B^{2}} \widetilde{A}_{a a^{\prime}}(B)\right|_{B=0}$ we obtain similar expressions.

\section{Appendix C. $U V$ limits of the gauged open string partition functions}

In this appendix we derive the $U V$ limit $t \rightarrow 0$ of the expressions Eqs. (2.17), (2.21), and (2.23). We determine this limit from the closed string expressions (2.29), (2.30), and (2.32), where this limit corresponds to the $I R$ limit $l \rightarrow \infty$. We use the product expansion for the $\theta$-functions

$$
\begin{aligned}
& \theta\left[\begin{array}{l}
\delta_{1} \\
\delta_{2}
\end{array}\right](z, \tau)=\sum_{n \in \mathbf{Z}} e^{\pi i \tau\left(n+\delta_{1}\right)^{2}} e^{2 \pi i\left(n+\delta_{1}\right)\left(z+\delta_{2}\right)} \\
& \quad=e^{2 \pi i \delta_{1}\left(z+\delta_{2}\right)} q^{\frac{\delta_{1}^{2}}{2}} \prod_{n=1}^{\infty}\left(1-q^{n}\right)\left(1+q^{n+\delta_{1}-\frac{1}{2}} e^{2 \pi i\left(z+\delta_{2}\right)}\right)\left(1+q^{n-\delta_{1}-\frac{1}{2}} e^{-2 \pi i\left(z+\delta_{2}\right)}\right)
\end{aligned}
$$


to derive the limits

$$
\lim _{\tau \rightarrow i \infty} \theta\left[\begin{array}{l}
\delta_{1} \\
\delta_{2}
\end{array}\right](i z, \tau) \rightarrow\left\{\begin{array}{lll}
1+2 q^{1 / 2} \cos (2 \pi i z) & , \delta_{1}=0 \quad, \quad \delta_{2}=0 \\
1-2 q^{1 / 2} \cos (2 \pi i z) & , \delta_{1}=0 \quad, \quad \delta_{2}=\frac{1}{2} \\
2 q^{1 / 8} \cos (\pi i z) & , \delta_{1}=\frac{1}{2}, & \delta_{2}=0 \\
-2 q^{1 / 8} \sin (\pi i z) & , \delta_{1}=\frac{1}{2}, & \delta_{2}=\frac{1}{2}
\end{array}\right.
$$

Furthermore we notify $\eta(\tau) \rightarrow q^{1 / 24}$, and the useful relations:

$$
\begin{aligned}
\cos \left(i \pi v_{a}^{j}\right) & =\cosh \left(\pi v_{a}^{j}\right)=\cos \left(\phi_{a}^{j}\right)=\frac{n_{a}^{j} R_{1}^{j}+m_{a}^{j} R_{2}^{j} \cos \alpha^{j}}{\mathcal{V}_{a}^{j}} \\
\sin \left(i \pi v_{a}^{j}\right) & =i \sinh \left(\pi v_{a}^{j}\right)=\sin \left(\phi_{a}^{j}\right)=\frac{m_{a}^{j} R_{2}^{j}}{\mathcal{V}_{a}^{j}} \sin \alpha^{j}, \\
\cot \left(i \pi v_{a}^{j}\right) & =-i \operatorname{coth}\left(\pi v_{a}^{j}\right)=\cot \left(\phi_{a}^{j}\right)=\frac{n_{a}^{j} R_{1}^{j}+m_{a}^{j} R_{2}^{j} \cos \alpha^{j}}{m_{a}^{j} R_{2}^{j} \sin \alpha^{j}} \\
\cos \left(i \pi v_{a b}^{j}\right) & =\cos \left(\phi_{b}^{j}-\phi_{a}^{j}\right) \\
& =\frac{1}{\mathcal{V}_{a}^{j} \mathcal{V}_{b}^{j}}\left[\left(R_{1}^{j}\right)^{2} n_{a}^{j} n_{b}^{j}+\left(R_{2}^{j}\right)^{2} m_{a}^{j} m_{b}^{j}+R_{1}^{j} R_{2}^{j}\left(n_{a}^{j} m_{b}^{j}+n_{b}^{j} m_{a}^{j}\right) \cos \alpha^{j}\right] \\
& =\frac{1}{2} \frac{1}{\mathcal{V}_{a}^{j} \mathcal{V}_{b}^{j}} \frac{T_{2}^{j}}{U_{2}^{j}}\left[\left(n_{a}^{j}+m_{a}^{j} U^{j}\right)\left(n_{b}^{j}+m_{b}^{j} \bar{U}^{j}\right)+\left(n_{a}^{j}+m_{a}^{j} \bar{U}^{j}\right)\left(n_{b}^{j}+m_{b}^{j} U^{j}\right)\right] \\
\sin \left(i \pi v_{a b}^{j}\right) & =\sin \left(\phi_{b}^{j}-\phi_{a}^{j}\right)=R_{1}^{j} R_{2}^{j} \frac{n_{a}^{j} m_{b}^{j}-n_{b}^{j} m_{a}^{j}}{\mathcal{V}_{a}^{j} \mathcal{V}_{b}^{j}} \sin \alpha^{j}=T_{2}^{j} \frac{I_{a b}^{j}}{\mathcal{V}_{a}^{j} \mathcal{V}_{b}^{j}}, \\
e^{i\left(\phi_{b}^{j}-\phi_{a}^{j}\right)} & =\frac{R_{1}^{j} R_{2}^{j}}{\mathcal{V}_{a}^{j} \mathcal{V}_{b}^{j}}\left[\frac{R_{1}^{j}}{R_{2}^{j}} n_{a}^{j} n_{b}^{j}+\frac{R_{2}^{j}}{R_{1}^{j}} m_{a}^{j} m_{b}^{j}+\left(n_{a}^{j} m_{b}^{j}+n_{b}^{j} m_{a}^{j}\right) \cos \alpha^{j}+i I_{a b}^{j} \sin \alpha^{j}\right]
\end{aligned}
$$

with

$$
\mathcal{V}_{a}^{j}=\sqrt{\frac{T_{2}^{j}}{U_{2}^{j}}\left|n_{a}^{j}+m_{a}^{j} U^{j}\right|^{2}}=\sqrt{\left(n_{a}^{j}\right)^{2}\left(R_{1}^{j}\right)^{2}+\left(m_{a}^{j}\right)^{2}\left(R_{2}^{j}\right)^{2}+2 n_{a}^{j} m_{a}^{j} R_{1}^{j} R_{2}^{j} \cos \alpha^{j}}
$$

describing the volume of brane $a$, which wraps the torus $T_{2}^{j}$ with wrapping numbers $\left(n_{a}^{j}, m_{a}^{j}\right)$. The above expressions are written down for an $A$ torus, i.e. the lattice basis vector $\vec{e}_{1}$ is aligned along the $Y^{2 i-1}$ axis. However, these expressions may be also used for a $\mathrm{B}$ type lattice after properly redefining the brane wrapping numbers $\left(n_{a}, m_{a}\right)$ into $\left(\widetilde{n}_{a}, \widetilde{m}_{a}\right)$. The latter are determined by the relation of the $A$-lattice basis vectors $\vec{e}_{1}, \vec{e}_{2}$ to the $B$-lattice basis vectors $\vec{e}_{1}^{\prime}, \vec{e}_{2}^{\prime}$. The same applies for the case of tilted tori. 
In the following we shall consider the cases $d=2,3$, appropriate for intersecting $D 6$ branes. For the $R$-sector $\vec{\delta}=\left(\frac{1}{2}, 0\right)$, we obtain in the limit $l \rightarrow \infty$ :

$$
\begin{aligned}
& \left.l^{-1} \widetilde{A}_{a b}^{d=3}(B)\right|_{R} \rightarrow I_{a b} \beta_{a b} \cot \left(\pi \epsilon_{a b}\right) \prod_{j=1}^{3} \cot \left(i \pi v_{a b}^{j}\right) \\
& =\prod_{j=1}^{3}\left[\frac{R_{1}^{j}}{R_{2}^{j}} n_{a}^{j} n_{b}^{j}+\frac{R_{2}^{j}}{R_{1}^{j}} m_{a}^{j} m_{b}^{j}+\left(n_{a}^{j} m_{b}^{j}+n_{b}^{j} m_{a}^{j}\right) \cos \alpha^{j}\right] \frac{1}{\sin \alpha^{j}}, \\
& \left.l^{-1} \widetilde{A}_{a b}^{d=2}(B)\right|_{R} \rightarrow I_{a b}^{1} I_{a b}^{2} V_{a}^{3} \beta_{a b} \cot \left(\pi \epsilon_{a b}\right) \prod_{j=1}^{2} \cot \left(i \pi v_{a b}^{j}\right) \\
& =V_{a}^{3} \prod_{j=1}^{2}\left[\frac{R_{1}^{j}}{R_{2}^{j}} n_{a}^{j} n_{b}^{j}+\frac{R_{2}^{j}}{R_{1}^{j}} m_{a}^{j} m_{b}^{j}+\left(n_{a}^{j} m_{b}^{j}+n_{b}^{j} m_{a}^{j}\right) \cos \alpha^{j}\right] \frac{1}{\sin \alpha^{j}} . \\
& \left.l^{-1} \quad \widetilde{A}_{a a^{\prime}}^{d=3}(B)\right|_{R} \rightarrow I_{a a^{\prime}} \beta_{a a^{\prime}} \cot \left(\pi \epsilon_{a a^{\prime}}\right) \prod_{j=1}^{3} \cot \left(i \pi v_{a a^{\prime}}^{j}\right) \\
& =\left(1-\pi^{2} q_{a} q_{a^{\prime}} B^{2}\right) \\
& \times \prod_{j=1}^{3}\left[\frac{R_{1}^{j}}{R_{2}^{j}} n_{a}^{j} n_{a^{\prime}}^{j}+\frac{R_{2}^{j}}{R_{1}^{j}} m_{a}^{j} m_{a^{\prime}}^{j}+\left(n_{a}^{j} m_{a^{\prime}}^{j}+n_{a^{\prime}}^{j} m_{a}^{j}\right) \cos \alpha^{j}\right] \frac{1}{\sin \alpha^{j}}, \\
& \left.l^{-1} \quad \widetilde{A}_{a a^{\prime}}^{d=2}(B)\right|_{R} \rightarrow\left(1-\pi^{2} q_{a} q_{a^{\prime}} B^{2}\right) I_{a a^{\prime}}^{1} I_{a a^{\prime}}^{2} V_{a}^{3} \\
& \times \prod_{j=1}^{2}\left[\frac{R_{1}^{j}}{R_{2}^{j}} n_{a}^{j} n_{a^{\prime}}^{j}+\frac{R_{2}^{j}}{R_{1}^{j}} m_{a}^{j} m_{a^{\prime}}^{j}+\left(n_{a}^{j} m_{a^{\prime}}^{j}+n_{a^{\prime}}^{j} m_{a}^{j}\right) \cos \alpha^{j}\right] \frac{1}{\sin \alpha^{j}} \\
& \left.l^{-1} \widetilde{M}_{a, \Omega \mathcal{R} \theta^{k} a}^{k ; j=3}(B)\right|_{R} \rightarrow-4 \beta_{a} \cot \left(\frac{\pi \epsilon_{a}}{2}\right) I_{a, \Omega \mathcal{R} \theta^{k} a}^{k} \prod_{j=1}^{3} 2^{\delta_{j}} \cot \left(i \pi v_{a}^{k ; j}\right) \\
& =-8 i I_{a, \Omega \mathcal{R} \theta^{k}}^{k} \prod_{j=1}^{3} 2^{\delta_{j}} \operatorname{coth}\left(\pi v_{a}^{k ; j}\right), \\
& \left.l^{-1} \widetilde{M}_{a, \Omega \mathcal{R} \theta^{k} a}^{k ; d=2}(B)\right|_{R} \rightarrow-2^{4-\mu} \beta_{a} \cot \left(\frac{\pi \epsilon_{a}}{2}\right) V_{O 6_{k}}^{i} \prod_{j=1}^{2} I_{a, \Omega \mathcal{R} \theta^{k} a}^{k ; j} \cot \left(i \pi v_{a}^{k ; j}\right) \\
& =2^{5-\mu} V_{O 6_{k}}^{i} \prod_{j=1}^{2} 2^{\delta_{j}} I_{a, \Omega \mathcal{R} \theta^{k} a}^{k ; j} \operatorname{coth}\left(\pi v_{a}^{k ; j}\right) .
\end{aligned}
$$


For the NS-sectors $\vec{\delta}=(0,0),\left(0, \frac{1}{2}\right)$ we obtain:

$$
\begin{aligned}
&\left.l^{-1} \widetilde{A}_{a b}^{d=3}(B)\right|_{N S} \rightarrow-\frac{i}{2} I_{a b} \frac{1}{\sinh \left(\pi v_{a b}^{1}\right) \sinh \left(\pi v_{a b}^{2}\right) \sinh \left(\pi v_{a b}^{3}\right)} \\
& \times\left\{\frac{1}{\sqrt{1+\pi^{2} q_{a}^{2} B^{2}}}+\frac{1}{2} \sqrt{1+\pi^{2} q_{a}^{2} B^{2}}\left[-1+\cosh \left(2 \pi v_{a b}^{1}\right)+\cosh \left(2 \pi v_{a b}^{2}\right)+\cosh \left(2 \pi v_{a b}^{3}\right)\right]\right\}, \\
&\left.l^{-1} \widetilde{A}_{a b}^{d=2}(B)\right|_{N S} \rightarrow \frac{1}{2} I_{a b}^{1} I_{a b}^{2} V_{a}^{3} \frac{1}{\sinh \left(\pi v_{a b}^{1}\right) \sinh \left(\pi v_{a b}^{2}\right)} \\
& \times\left\{\frac{1}{\sqrt{1+\pi^{2} q_{a}^{2} B^{2}}}+\frac{1}{2} \sqrt{1+\pi^{2} q_{a}^{2} B^{2}}\left[\cosh \left(2 \pi v_{a b}^{1}\right)+\cosh \left(2 \pi v_{a b}^{2}\right)\right]\right\} .
\end{aligned}
$$

Furthermore, for gauged open string partition function with both string ends carrying charges $q_{a}, q_{a^{\prime}}$ of the gauge group $G_{a}$, we determine:

$$
\begin{aligned}
\left.l^{-1} \widetilde{A}_{a a^{\prime}}^{d=3}(B)\right|_{N S} & \rightarrow-\frac{i}{2} \frac{I_{a a^{\prime}}}{\sinh \left(\pi v_{a a^{\prime}}^{1}\right) \sinh \left(\pi v_{a a^{\prime}}^{2}\right) \sinh \left(\pi v_{a a^{\prime}}^{3}\right)} \\
& \times\left\{\frac{\left(1-\pi^{2} q_{a} q_{a^{\prime}} B^{2}\right)^{2}}{\sqrt{1+\pi^{2} q_{a}^{2} B^{2}} \sqrt{1+\pi^{2} q_{a^{\prime}}^{2} B^{2}}}+\frac{1}{2} \sqrt{1+\pi^{2} q_{a}^{2} B^{2}} \sqrt{1+\pi^{2} q_{a^{\prime}}^{2} B^{2}}\right. \\
& \left.\times\left[-1+\cosh \left(2 \pi v_{a a^{\prime}}^{1}\right)+\cosh \left(2 \pi v_{a a^{\prime}}^{2}\right)+\cosh \left(2 \pi v_{a a^{\prime}}^{3}\right)\right]\right\}, \\
\left.l^{-1} \widetilde{A}_{a a^{\prime}}^{d=2}(B)\right|_{N S} & \rightarrow \frac{1}{2} V_{a}^{3} \frac{I_{a a^{\prime}}^{1} I_{a a^{\prime}}^{2}}{\sinh \left(\pi v_{a a^{\prime}}^{1}\right) \sinh \left(\pi v_{a a^{\prime}}^{2}\right)}\left\{\frac{\left(1-\pi^{2} q_{a} q_{a^{\prime}} B^{2}\right)^{2}}{\sqrt{1+\pi^{2} q_{a}^{2} B^{2}} \sqrt{1+\pi^{2} q_{a^{\prime}}^{2} B^{2}}}\right. \\
& \left.+\frac{1}{2} \sqrt{1+\pi^{2} q_{a}^{2} B^{2}} \sqrt{1+\pi^{2} q_{a^{\prime}}^{2} B^{2}}\left[\cosh \left(2 \pi v_{a a^{\prime}}^{1}\right)+\cosh \left(2 \pi v_{a a^{\prime}}^{2}\right)\right]\right\}, \\
\left.l^{-1} \widetilde{A}_{a a^{\prime}}^{d=0}(B)\right|_{N S} & \rightarrow \prod_{j=1}^{d} V_{a}^{j} \\
& \times\left\{\frac{1}{2} \frac{\pi^{2}\left(q_{a}+q_{a^{\prime}}\right)^{2} B^{2}}{\sqrt{1+\pi^{2} q_{a}^{2} B^{2}} \sqrt{1+\pi^{2} q_{a^{\prime}}^{2} B^{2}}}-\sqrt{1+\pi^{2} q_{a}^{2} B^{2}} \sqrt{1+\pi^{2} q_{a^{\prime}}^{2} B^{2}}\right\} .
\end{aligned}
$$


And for the gauged Möbius partition function we obtain:

$$
\begin{aligned}
& \left.l^{-1} \widetilde{M}_{a, \Omega \mathcal{R} \theta^{k} a}^{k ; d=3}(B)\right|_{N S} \rightarrow-4 i I_{a, \Omega \mathcal{R} \theta^{k} a}^{k} \frac{1}{\sinh \left(\pi v_{a}^{k ; 1}\right) \sinh \left(\pi v_{a}^{k ; 2}\right) \sinh \left(\pi v_{a}^{k ; 3}\right)} \\
& \times\left\{\frac{1}{\sqrt{1+\pi^{2} q_{a}^{2} B^{2}}}+\frac{1}{2} \sqrt{1+\pi^{2} q_{a}^{2} B^{2}}\left[-1+\cosh \left(2 \pi v_{a}^{k ; 1}\right)+\cosh \left(2 \pi v_{a}^{k ; 2}\right)+\cosh \left(2 \pi v_{a}^{k ; 3}\right)\right]\right\}, \\
& \left.l^{-1} \widetilde{M}_{a, \Omega \mathcal{R} \theta^{k} a}^{k ; d=2}(B)\right|_{N S} \rightarrow-16 V_{O 6_{k}}^{i} I_{a, \Omega \mathcal{R} \theta^{k} a}^{k ; 1} I_{a, \Omega \mathcal{R} \theta^{k} a}^{k ; 2} \frac{1}{\sinh \left(\pi v_{a}^{k ; 1}\right) \sinh \left(\pi v_{a}^{k ; 2}\right)} \\
& \times\left\{\frac{1}{\sqrt{1+\pi^{2} q_{a}^{2} B^{2}}}+\frac{1}{2} \sqrt{1+\pi^{2} q_{a}^{2} B^{2}}\left[\cosh \left(2 \pi v_{a}^{k ; 1}\right)+\cosh \left(2 \pi v_{a}^{k ; 2}\right)\right]\right\} .
\end{aligned}
$$

Obviously, the $R$-sector (Eqs. (C.5), (C.6) and (C.7)) shows a very simple dependence on the magnetic field $B$. In fact, only $\left.\widetilde{A}_{a a^{\prime}}^{d}(B)\right|_{R}$ shows a quadratic order in $B$. However, with taking into account the condition (3.24) we conclude, that there are no tadpole contributions in the $R$-sector for anomaly free gauge groups. This fact is not true for the $N S$-sector, which has generically a functional dependence on the magnetic field resembling the relevant Schwinger expressions from field theory. Note that for the $\mathrm{N}=2$ sector, in the case of $v_{a b}^{1}=-v_{a b}^{1}= \pm 1 / 2$, this functional behaviour boils down to the expressions for the $\mathbf{Z}_{2}$ orientifold presented in [18]. To discuss the possible tadpole contributions in subsection 3.4. we only need from Eqs. (C.8), (C.9) and (C.10) the second order parts in B, which take the form:

$$
\begin{aligned}
\left.l^{-1} \quad \widetilde{A}_{a b}^{d=3}(B)\right|_{N S} & \rightarrow-i I_{a b}\left\{\prod_{j=1}^{3} \operatorname{coth}\left(\pi v_{a b}^{j}\right)\right. \\
& \left.+\frac{1}{2} \pi^{2} q_{a}^{2} B^{2}\left[\prod_{j=1}^{3} \operatorname{coth}\left(\pi v_{a b}^{j}\right)-\prod_{j=1}^{3} \frac{1}{\sinh \left(\pi v_{a b}^{j}\right)}\right]\right\}+\mathcal{O}\left(B^{4}\right), \\
\left.l^{-1} \quad \widetilde{A}_{a b}^{d=2}(B)\right|_{N S} & \rightarrow I_{a b}^{1} I_{a b}^{2} V_{a}^{3}\left\{\prod_{j=1}^{2} \operatorname{coth}\left(\pi v_{a b}^{j}\right)\right. \\
& \left.+\frac{1}{2} \pi^{2} q_{a}^{2} B^{2}\left[\prod_{j=1}^{2} \operatorname{coth}\left(\pi v_{a b}^{j}\right)-\prod_{j=1}^{2} \frac{1}{\sinh \left(\pi v_{a b}^{j}\right)}\right]\right\}+\mathcal{O}\left(B^{4}\right) .
\end{aligned}
$$

These identities assume the supersymmetry conditions $v_{a b}^{1}+v_{a b}^{2}+v_{a b}^{3}=0$ for $A_{a b}^{d=3}$ and $v_{a b}^{1}+v_{a b}^{2}=0$ for $A_{a b}^{d=2}$, respectively. Furthermore for the annulus between one brane $a$ 
from stack $a$ and an other brane $a^{\prime}$ from its mirror:

$$
\begin{aligned}
l^{-1} & \left.\widetilde{A}_{a a^{\prime}}^{d=3}(B)\right|_{N S} \rightarrow-i I_{a a^{\prime}}\left\{\prod_{j=1}^{3} \operatorname{coth}\left(\pi v_{a a^{\prime}}^{j}\right)\right. \\
& \left.+\frac{1}{2} \pi^{2} B^{2}\left[\left(q_{a}^{2}+q_{a^{\prime}}^{2}\right) \prod_{j=1}^{3} \operatorname{coth}\left(\pi v_{a a^{\prime}}^{j}\right)-\left(q_{a}+q_{a^{\prime}}\right)^{2} \prod_{j=1}^{3} \frac{1}{\sinh \left(\pi v_{a a^{\prime}}^{j}\right)}\right]\right\}+\mathcal{O}\left(B^{4}\right), \\
l^{-1} & \left.\widetilde{A}_{a a^{\prime}}^{d=2}(B)\right|_{N S} \rightarrow I_{a a^{\prime}}^{1} I_{a a^{\prime}}^{2} V_{a}^{3}\left\{\prod_{j=1}^{2} \operatorname{coth}\left(\pi v_{a a^{\prime}}^{j}\right)\right. \\
& \left.+\frac{1}{2} \pi^{2} B^{2}\left[\left(q_{a}^{2}+q_{a^{\prime}}^{2}\right) \prod_{j=1}^{2} \operatorname{coth}\left(\pi v_{a a^{\prime}}^{j}\right)-\left(q_{a}+q_{a^{\prime}}\right)^{2} \prod_{j=1}^{2} \frac{1}{\sinh \left(\pi v_{a a^{\prime}}^{j}\right)}\right]\right\}+\mathcal{O}\left(B^{4}\right), \\
l^{-1} & \left.\widetilde{A}_{a a^{\prime}}^{d=0}(B)\right|_{N S} \rightarrow\left(-1+B^{2} \pi^{2} q_{a} q_{a^{\prime}}\right) \prod_{j=1}^{3} V_{a}^{j} \\
& =\left(-1+B^{2} \pi^{2} q_{a} q_{a^{\prime}}\right) \prod_{j=1}^{3}\left[\frac{R_{1}^{j}}{R_{2}^{j}}\left(n_{a}^{j}\right)^{2}+\frac{R_{2}^{j}}{R_{1}^{j}}\left(m_{a}^{j}\right)^{2}+2 n_{a}^{j} m_{a}^{j} \cos \alpha^{j}\right] \frac{1}{\sin \alpha^{j}}+\mathcal{O}\left(B^{4}\right) .
\end{aligned}
$$

Again these identities rely on the supersymmetry conditions $v_{a b}^{1}+v_{a b}^{2}+v_{a b}^{3}=0$ for $A_{a b}^{d=3}$ and $v_{a b}^{1}+v_{a b}^{2}=0$ for $A_{a b}^{d=2}$, respectively. Finally, for the Möbius function the lowest expansion in $F$ of (C.10) yields in case of $\mathrm{N}=1$ and $\mathrm{N}=2$ supersymmetry, respectively:

$$
\begin{aligned}
& \left.l^{-1} \widetilde{M}_{a, \Omega \mathcal{R} \theta^{k} a}^{k ; d=3}(B)\right|_{N S} \rightarrow 8 i I_{a, \Omega \mathcal{R} \theta^{k} a}^{k}\left\{\prod_{j=1}^{3} \operatorname{coth}\left(\pi v_{a}^{k ; j}\right)\right. \\
& \left.+\frac{1}{2} \pi^{2} q_{a}^{2} B^{2}\left[\prod_{j=1}^{3} \operatorname{coth}\left(\pi v_{a}^{k ; j}\right)-\prod_{j=1}^{3} \frac{1}{\sinh \left(\pi v_{a}^{k ; j}\right)}\right]\right\}+\mathcal{O}\left(B^{4}\right) \\
& \left.l^{-1} \widetilde{M}_{a, \Omega \mathcal{R} \theta^{k} a}^{k ; d=2}(B)\right|_{N S} \rightarrow-32 I_{a, \Omega \mathcal{R} \theta^{k} a}^{k ; 1} I_{a, \Omega \mathcal{R} \theta^{k} a}^{k ; 2} V_{O 6_{k}}^{i}\left\{\prod_{j=1}^{2} \operatorname{coth}\left(\pi v_{a}^{k ; j}\right)\right. \\
& \left.+\frac{1}{2} \pi^{2} q_{a}^{2} B^{2}\left[\prod_{j=1}^{2} \operatorname{coth}\left(\pi v_{a}^{k ; j}\right)-\prod_{j=1}^{2} \frac{1}{\sinh \left(\pi v_{a}^{k ; j}\right)}\right]\right\}+\mathcal{O}\left(B^{4}\right) .
\end{aligned}
$$

Of course, in the case of supersymmetry, the $R$ and $N S$ vacuum tadpoles are the same up to a minus sign as a result of vanishing vacuum partition function. However, this is no longer the case for our open string partition functions depending on the space-time gauge field $B$. For the case $d=3$, describing the $\mathrm{N}=1$ sectors, the total NS tadpole contributions 
at order $B^{2}$ can be read off from the results (C.11), (C.12) and (C.13). Note, that these expressions are the same as the one given in Eqs. (3.40), (3.41), and (3.42). This is due to the general identity

$$
\begin{aligned}
& -\operatorname{coth}\left(\pi v^{1}\right) \operatorname{coth}\left(\pi v^{2}\right) \operatorname{coth}\left(\pi v^{3}\right)+\frac{1}{\sinh \left(\pi v^{1}\right) \sinh \left(\pi v^{2}\right) \sinh \left(\pi v^{3}\right)} \\
& =\operatorname{coth}\left(\pi v^{1}\right)+\operatorname{coth}\left(\pi v^{2}\right)+\operatorname{coth}\left(\pi v^{3}\right)
\end{aligned}
$$

valid for the supersymmetric case $v^{1}+v^{2}+v^{3}=0$. Similar for the $\mathrm{N}=2$ sector contributions $d=2$, derived in (C.11), (C.12) and (C.13), we may use the identity

$$
-\operatorname{coth}\left(\pi v^{1}\right) \operatorname{coth}\left(\pi v^{2}\right)+\frac{1}{\sinh \left(\pi v^{1}\right) \sinh \left(\pi v^{2}\right)}=1
$$

for the case $v^{1}+v^{2}=0$ to make contact with the UV-divergent expressions from subsection 3.3 . 


\section{References}

[1] M. Berkooz, M.R. Douglas and R.G. Leigh, "Branes Intersecting at Angles," Nucl. Phys. B 480 (1996) 265, arXiv:hep-th/9606139;

R. Blumenhagen, L. Görlich, B. Körs and D. Lüst, "Noncommutative compactifications of type I strings on tori with magnetic background flux," JHEP 0010, 006 (2000) arXiv:hep-th/0007024;

C. Angelantonj, I. Antoniadis, E. Dudas and A. Sagnotti, "Type I Strings on Magne-

tized Orbifolds and Brane Transmutation", Phys. Lett. B 489 (2000) 223, arXiv:hepth/0007090|;

C. Angelantonj and A. Sagnotti, "Type I Vacua and Brane Transmutation", hepth/0010279;

G. Aldazabal, S. Franco, L.E. Ibanez, R. Rabadan and A.M. Uranga, "D = 4 chiral string compactifications from intersecting branes," J. Math. Phys. 42, 3103 (2001) arXiv:hep-th/0011073;

G. Aldazabal, S. Franco, L.E. Ibanez, R. Rabadan, and A.M. Uranga, "Intersecting Brane Worlds", JHEP 0102 (2001) 047, [arXiv:hep-ph/0011132;

R. Blumenhagen, B. Körs and D. Lüst, "Type I strings with F- and B-flux," JHEP 0102, 030 (2001) arXiv:hep-th/0012156;

L.E. Ibanez, F. Marchesano, R. Rabadan, "Getting just the Standard Model at Intersecting Branes", JHEP 0111 (2001) 002, arXiv:hep-th/0105155];

S. Förste, G. Honecker and R. Schreyer, "Orientifolds with branes at angles," JHEP 0106, 004 (2001) arXiv:hep-th/0105208;

R. Rabadan, "Branes at Angles, Torons, Stability and Supersymmetry", Nucl. Phys. B 620 (2002) 152, arXiv:hep-th/0107036;

R. Blumenhagen, B. Körs, D. Lüst and T. Ott, "The standard model from stable intersecting brane world orbifolds," Nucl. Phys. B 616, 3 (2001) arXiv:hep-th/0107138;

M. Cvetic, G. Shiu and A.M. Uranga, "Chiral four-dimensional $N=1$ supersymmetric type IIA orientifolds from intersecting D6-branes," Nucl. Phys. B 615, 3 (2001) arXiv:hep-th/0107166;

D. Bailin, G.V. Kraniotis and A. Love, "New Standard-like Models from Intersecting D4-Branes", hep-th/0208103; "Standard-like models from intersecting D5-branes", hep-th/0210227;

D. Cremades, L.E. Ibanez and F. Marchesano, "SUSY quivers, intersecting branes and the modest hierarchy problem," JHEP 0207, 009 (2002) arXiv:hep-th/0201205;

R. Blumenhagen, B. Körs and D. Lüst, "Moduli stabilization for intersecting brane worlds in type 0' string theory," Phys. Lett. B 532, 141 (2002) arXiv:hep-th/0202024; D. Cremades, L.E. Ibanez and F. Marchesano, "Intersecting Brane Models of Particle Physics and the Higgs Mechanism", JHEP 0207 (2002) 022, arXiv:hep-th/0203160; 
C. Kokorelis, "GUT Model Hierarchies from Intersecting Branes", JHEP 0208 (2002) 018, arXiv:hep-th/0203187; "Deformed Intersecting D6-Brane GUTS I", hepth/0209202; "Deformed Intersecting D6-Brane GUTS II", hep-th/0210200;

R. Blumenhagen, V. Braun, B. Körs and D. Lüst, "Orientifolds of K3 and CalabiYau manifolds with intersecting D-branes," JHEP 0207, 026 (2002) arXiv:hepth/0206038; "The standard model on the quintic," hep-th/0210083;

A.M. Uranga, "Local models for intersecting brane worlds", JHEP 0212 (2002) 058, arXiv:hep-th/0208014;

G. Pradisi, "Magnetized (Shift-)Orientifolds", hep-th/0210088;

R. Blumenhagen, L. Görlich and T. Ott, "Supersymmetric Intersecting Branes on the Type IIA $T^{6} / Z_{4}$ orientifold", JHEP 0301 (2003) 021, hep-th/0211059;

M. Cvetic, I. Papadimitriou and G. Shiu, "Supersymmetric Three Family SU(5) Grand Unified Models from Type IIA Orientifolds with Intersecting D6-Branes", hepth/0212177.

[2] D. Cremades, L.E. Ibanez and F. Marchesano, "SUSY quivers, intersecting branes and the modest hierarchy problem," JHEP 0207, 009 (2002) [arXiv:hep-th/0201205].

[3] M. Cvetic, P. Langacker and G. Shiu, "Phenomenology of a three-family standard-like string model," Phys. Rev. D 66, 066004 (2002) [arXiv:hep-ph/0205252];

G. Shiu and S.H. Tye, "TeV scale superstring and extra dimensions," Phys. Rev. D 58, 106007 (1998) arXiv:hep-th/9805157.

[4] V. S. Kaplunovsky, "One Loop Threshold Effects In String Unification," Nucl. Phys. B 307, 145 (1988) [Erratum-ibid. B 382, 436 (1992)] [arXiv:hep-th/9205068].

[5] L.J. Dixon, V. Kaplunovsky and J. Louis, "Moduli Dependence Of String Loop Corrections To Gauge Coupling Constants," Nucl. Phys. B 355, 649 (1991).

[6] I. Antoniadis, K.S. Narain and T.R. Taylor, "Higher genus string corrections to gauge couplings," Phys. Lett. B 267, 37 (1991);

I. Antoniadis, E. Gava and K.S. Narain, "Moduli corrections to gauge and gravitational couplings in four-dimensional superstrings," Nucl. Phys. B 383, 93 (1992) arXiv:hep-th/9204030;

I. Antoniadis, E. Gava, K.S. Narain and T.R. Taylor, "Superstring threshold corrections to Yukawa couplings," Nucl. Phys. B 407, 706 (1993) arXiv:hep-th/9212045;

P. Mayr and S. Stieberger, "Threshold corrections to gauge couplings in orbifold compactifications," Nucl. Phys. B 407, 725 (1993) arXiv:hep-th/9303017]; "Dilaton, antisymmetric tensor and gauge fields in string effective theories at the one loop level," Nucl. Phys. B 412, 502 (1994) arXiv:hep-th/9304055]; "Moduli dependence of one loop gauge couplings in $(0,2)$ compactifications," Phys. Lett. B 355, 107 (1995) arXiv:hep-th/9504129;

E. Kiritsis and C. Kounnas, "Infrared regularization of superstring theory and the one 
loop calculation of coupling constants," Nucl. Phys. B 442, 472 (1995) arXiv:hepth/9501020 ;

E. Kiritsis, C. Kounnas, P.M. Petropoulos and J. Rizos, "Universality properties of $\mathrm{N}=2$ and $\mathrm{N}=1$ heterotic threshold corrections," Nucl. Phys. B 483, 141 (1997) arXiv:hep-th/9608034;

J.A. Harvey and G.W. Moore, "Algebras, BPS States, and Strings," Nucl. Phys. B 463, 315 (1996) arXiv:hep-th/9510182;

H.P. Nilles and S. Stieberger, "String unification, universal one-loop corrections and strongly coupled heterotic string theory," Nucl. Phys. B 499, 3 (1997) arXiv:hepth/9702110;

S. Stieberger, "(0,2) heterotic gauge couplings and their M-theory origin," Nucl. Phys. B 541, 109 (1999) arXiv:hep-th/9807124.

[7] L.E. Ibanez, D. Lüst and G.G. Ross, "Gauge coupling running in minimal $S U(3) \times$ $S U(2) \times U(1)$ superstring unification," Phys. Lett. B 272, 251 (1991) arXiv:hepth/9109053;

L.E. Ibanez and D. Lüst, "Duality anomaly cancellation, minimal string unification and the effective low-energy Lagrangian of 4-D strings," Nucl. Phys. B 382, 305 (1992) arXiv:hep-th/9202046;

P. Mayr, H.P. Nilles and S. Stieberger, "String unification and threshold corrections," Phys. Lett. B 317, 53 (1993) arXiv:hep-th/9307171];

H.P. Nilles and S. Stieberger, "How to Reach the Correct $\sin ^{2} \theta_{W}$ and $\alpha_{S}$ in String Theory," Phys. Lett. B 367, 126 (1996) arXiv:hep-th/9510009; "String unification, universal one-loop corrections and strongly coupled heterotic string theory," Nucl. Phys. B 499, 3 (1997) arXiv:hep-th/9702110.

[8] Work in progress.

[9] J. Gomis, T. Mehen and M.B. Wise, "Quantum field theories with compact noncommutative extra dimensions," JHEP 0008, 029 (2000) arXiv:hep-th/0006160;

Z. Guralnik, R.C. Helling, K. Landsteiner and E. Lopez, "Perturbative instabilities on the non-commutative torus, Morita duality and twisted boundary conditions," JHEP 0205, 025 (2002) arXiv:hep-th/0204037.

[10] D. Lüst and S. Stieberger, to appear.

[11] D.M. Ghilencea, H.P. Nilles and S. Stieberger, "Divergences in Kaluza-Klein models and their string regularization," New J. Phys. 4, 15 (2002) arXiv:hep-th/0108183.

[12] R. Blumenhagen, L. Görlich and B. Körs, "Supersymmetric 4D orientifolds of type IIA with D6-branes at angles," JHEP 0001, 040 (2000) arXiv:hep-th/9912204.

[13] S. Förste, G. Honecker and R. Schreyer, "Supersymmetric $\mathbf{Z}_{N} \times \mathbf{Z}_{M}$ orientifolds in 4D with D-branes at angles," Nucl. Phys. B 593, 127 (2001) arXiv:hep-th/0008250;

R. Schreyer, PhD thesis, Bonn University 2001. 
[14] R. Blumenhagen, L. Görlich, B. Körs and D. Lüst, "Noncommutative compactifications of type I strings on tori with magnetic background flux," JHEP 0010, 006 (2000) arXiv:hep-th/0007024.

[15] R. Blumenhagen, B. Körs, D. Lüst and T. Ott, "The standard model from stable intersecting brane world orbifolds," Nucl. Phys. B 616, 3 (2001) arXiv:hep-th/0107138.

[16] M. Cvetic, G. Shiu and A.M. Uranga, "Chiral four-dimensional N = 1 supersymmetric type IIA orientifolds from intersecting D6-branes," Nucl. Phys. B 615, 3 (2001) arXiv:hep-th/0107166.

[17] R. Blumenhagen, L. Görlich and T. Ott, "Supersymmetric intersecting branes on the type IIA $T^{6} / \mathbf{Z}_{4}$ orientifold," hep-th/0211059.

[18] C. Bachas and C. Fabre, "Threshold Effects in Open-String Theory," Nucl. Phys. B 476, 418 (1996) arXiv:hep-th/9605028.

[19] I. Antoniadis, C. Bachas and E. Dudas, "Gauge couplings in four-dimensional type I string orbifolds," Nucl. Phys. B 560, 93 (1999) arXiv:hep-th/9906039.

[20] A. Abouelsaood, C.G. Callan, C.R. Nappi and S.A. Yost, "Open Strings In Background Gauge Fields," Nucl. Phys. B 280, 599 (1987); "String Loop Corrections To Beta Functions," Nucl. Phys. B 288, 525 (1987).

[21] C. Bachas and M. Porrati, "Pair Creation Of Open Strings In An Electric Field," Phys. Lett. B 296, 77 (1992) arXiv:hep-th/9209032.

[22] F. Ardalan, H. Arfaei and M.M. Sheikh-Jabbari, "Noncommutative geometry from strings and branes," JHEP 9902, 016 (1999) [arXiv:hep-th/9810072].

[23] R. Blumenhagen, L. Görlich, B. Körs and D. Lüst, "Asymmetric orbifolds, noncommutative geometry and type I string vacua," Nucl. Phys. B 582, 44 (2000) arXiv:hepth/0003024].

[24] R. Blumenhagen, B. Körs and D. Lüst, "Type I strings with F- and B-flux," JHEP 0102, 030 (2001) arXiv:hep-th/0012156.

[25] M. Cvetic, I. Papadimitriou and G. Shiu, "Supersymmetric Three Family SU(5) Grand Unified Models from Type IIA Orientifolds with Intersecting D6-Branes", hepth/0212177

[26] R. Blumenhagen, L. Görlich and B. Körs, "Supersymmetric orientifolds in 6D with D-branes at angles," Nucl. Phys. B 569, 209 (2000) arXiv:hep-th/9908130.

[27] S. Förste, G. Honecker and R. Schreyer, "Orientifolds with branes at angles," JHEP 0106, 004 (2001) arXiv:hep-th/0105208;

G. Honecker, PhD thesis, Bonn University 2002.

[28] A.N. Schellekens and N.P. Warner, "Anomalies, Characters And Strings," Nucl. Phys. B 287, 317 (1987); "Anomalies And Modular Invariance In String Theory," Phys. Lett. B 177, 317 (1986);

W. Lerche, "Elliptic Index And Superstring Effective Actions," Nucl. Phys. B 308, 102 (1988); 
W. Lerche, A.N. Schellekens and N.P. Warner, "Lattices And Strings," Phys. Rept. 177, 1 (1989).

[29] W. Lerche, B.E. Nilsson, A.N. Schellekens and N.P. Warner, "Anomaly Cancelling Terms From The Elliptic Genus," Nucl. Phys. B 299, 91 (1988).

[30] K. Foerger and S. Stieberger, "Higher derivative couplings and heterotic-type I duality in eight dimensions," Nucl. Phys. B 559, 277 (1999) arXiv:hep-th/9901020.

[31] I.S. Gradshteyn and I.M. Ryzhik, "Table of Integrals, Series and Products", Academic Press 1994.

[32] E.S. Fradkin and A.A. Tseytlin, "Nonlinear Electrodynamics From Quantized Strings," Phys. Lett. B 163, 123 (1985);

R.R. Metsaev and A.A. Tseytlin, "On Loop Corrections To String Theory Effective Actions," Nucl. Phys. B 298, 109 (1988);

A.A. Tseytlin, "Vector Field Effective Action In The Open Superstring Theory," Nucl. Phys. B 276, 391 (1986) [Erratum-ibid. B 291, 876 (1987)].

[33] J.A. Harvey and G.W. Moore, "Algebras, BPS States, and Strings," Nucl. Phys. B 463, 315 (1996) arXiv:hep-th/9510182.

[34] W. Lerche and S. Stieberger, "Prepotential, mirror map and F-theory on K3," Adv. Theor. Math. Phys. 2, 1105 (1998) [Erratum-ibid. 3, 1199 (1999)] arXiv:hepth/9804176].

[35] T. Friedmann and E. Witten, "Unification scale, proton decay, and manifolds of G(2) holonomy," hep-th/0211269.

[36] W. Lerche and S. Stieberger, "1/4 BPS states and non-perturbative couplings in N = 4 string theories," Adv. Theor. Math. Phys. 3, 1539 (1999) arXiv:hep-th/9907133.

[37] A.B. Hammou and J.F. Morales, "Fivebrane instantons and higher derivative couplings in type I theory," Nucl. Phys. B 573, 335 (2000) arXiv:hep-th/9910144.

[38] T.M. Apostol "Modular functions and Dirichlet series in number theory", Springer 1997.

[39] S. Stieberger and T.R. Taylor, "Non-Abelian Born-Infeld action and type I - heterotic duality. II: Nonrenormalization theorems," Nucl. Phys. B 648, 3 (2003) arXiv:hepth/0209064. 